\title{
COMMUTATION METHODS APPLIED TO THE mKdV-EQUATION
}

\author{
F. GESZTESY, W. SCHWEIGER, AND B. SIMON
}

Dedicated to Raphael Høegh-Krohn (1938-1988)

\begin{abstract}
An explicit construction of solutions of the modified Korteweg-de Vries equation given a solution of the (ordinary) Korteweg-de Vries equation is provided. Our theory is based on commutation methods (i.e., $N=1$ supersymmetry) underlying Miura's transformation that links solutions of the two evolution equations.
\end{abstract}

\section{INTRODUCTION}

In connection with the extensively studied Korteweg-de Vries (KdV-) equation

$$
\operatorname{KdV}(V):=V_{t}-6 V V_{x}+V_{x x x}=0, \quad(t, x) \in \mathbb{R}^{2},
$$

its cousins, the modified Korteweg-de Vries $\left(\mathrm{mKdV}_{ \pm^{-}}\right)$equations

$$
\mathrm{mKdV}_{ \pm}(\phi):=\phi_{t} \pm 6 \phi^{2} \phi_{x}+\phi_{x x x}=0, \quad(t, x) \in \mathbb{R}^{2},
$$

have also been investigated. In fact, $(1.2+)$ has been treated in some detail in the literature: See, e.g., $[12,51,67,68,69,74,111]$ for existence and uniqueness questions, $[58,105,107,113,114]$ for the derivation of the $N$-soliton solutions and $[94,108]$ for the general approach to $(1.2+)$ via inverse scattering techniques. The Lax pair for (1.2 \pm$)$ has been given in [107] and finally $(1.2 \pm)$ have been found to be subordinate to the AKNS-ZS-theory [4, 93] (cf. [34]). For more recent work on $(1.2+)$ see, e.g., $[11,13,70,118]$.

Surprisingly enough, apart from existence and uniqueness questions of solutions of $(1.2-)$ in $[12,51,68,69,74,111]$, no detailed study of $(1.2-)$ seems to have appeared until 1984 when Grosse [54] (see also [55]) derived the $N$-soliton solutions of (1.2-).

The present paper is devoted to a detailed investigation of real-valued solutions of the $\mathrm{mKdV}$ _-equation (1.2-) (from now on simply denoted by $\mathrm{mKdV}$ ).

Received by the editors January 15, 1990.

1980 Mathematics Subject Classification (1985 Revision). Primary 35G20, 76B25.

Key words and phrases. mKdV-equation, commutation methods, soliton-like solutions, periodic solutions, singular solutions.

The first author is a Max Kade Foundation Fellow and, with the third author, was partially supported by USNSF under Grant DMS-8801918.

The second author was supported by BMFT, Federal Republic of Germany. 
Our theory is based on Miura's fundamental relation [88]

$$
\operatorname{KdV}\left(\phi^{2} \pm \phi_{x}\right)=\left[2 \phi \pm \partial_{x}\right] \mathrm{mKdV}(\phi)
$$

that yields solutions $\phi^{2} \pm \phi_{x}$ of the $\mathrm{KdV}$-equation (1.1) given a solution $\phi$ of the $\mathrm{mKdV}$-equation $(1.2-)$. In view of the fact that $\mathrm{mKdV}$ has a higher nonlinearity than $\mathrm{KdV}$ and the latter has been studied so thoroughly in the literature, one would clearly like to invert that procedure, i.e., given a solution $V$ of (1.1) one would like to construct all solutions $\phi$ of $(1.2-)$ that are linked to each other via Miura's transformation

$$
V=\phi^{2}-\phi_{x} \quad\left(\text { resp. } V=\phi^{2}+\phi_{x}\right) .
$$

For smooth, bounded, and real-valued solutions $V, \phi$ this has been achieved in [50] from a theoretical point of view (see Theorem 7.9). Our main objective in this paper is to illustrate this method at work, i.e., to explicitly construct new classes of solutions of the $\mathrm{mKdV}$-equation such as soliton-like solutions, periodic solutions, solitons relative to periodic background solutions and to describe some of their properties. (Here soliton-like means solutions $V(\phi)$ of the $(\mathrm{m}) \mathrm{KdV}$-equation that approach time-independent (possibly different) asymptotic values $V_{ \pm}\left(\phi_{ \pm}\right) \in \mathbb{R}$ as $x \rightarrow \pm \infty$ and periodic always refers to spatially periodic solutions, i.e., for some $a>0$ :

$$
V(t, x+a)=V(t, x)(\phi(t, x+a)=\phi(t, x))
$$

for all $(t, x) \in \mathbb{R}^{2}$.)

Before describing the content of this paper in more detail we would like to explain the main ideas of [50] in an informal manner. Consider the Schrödinger operators

$$
H_{j}=-\partial_{x}^{2}+V_{j}, \quad j=1,2,
$$

in $L^{2}(\mathbb{R})$, where the $V_{j}$ are defined according to Miura's transformation (1.4), i.e.,

$$
V_{j}=\phi^{2}+(-1)^{j} \phi_{x}, \quad j=1,2 .
$$

(In order to simplify matters, $V_{j}, \phi$, and its partial derivatives are assumed to be real-valued, smooth, and bounded.) By inspection one verifies the factorizations

$$
H_{1}=A^{*} A, \quad H_{2}=A A^{*},
$$

where

$$
A=\partial_{x}+\phi .
$$

In particular, this forces $H_{j}$ to be nonnegative, i.e.,

$$
H_{j} \geq 0, \quad j=1,2 .
$$


In order to solve the Riccati-equation (1.6) for $\phi$ if, for instance, $V_{1}$ is given, one introduces the linearization

$$
\phi=-\psi_{0, x} / \psi_{0}
$$

where $\psi_{0}$ satisfies

$$
H_{1} \psi=0 \text { and hence } A \psi=0
$$

(the latter equation being equivalent to (1.10)) in the distributional sense. Equation (1.10) shows two things: First of all we need $\psi_{0}>0$ in order to get nonsingular $\phi$ 's. Secondly, $\phi$ will be uniquely determined by $V_{1}$ only if the equation $H_{1} \psi=0$ has a unique (up to multiple of constants) positive solution $\psi_{0}$. Otherwise, we expect a one-parameter family of solutions

$$
\phi_{\sigma}=-\partial_{x} \ln \psi_{\sigma},
$$

where

$$
\psi_{\sigma}=(1-\sigma) \psi_{-}+(1+\sigma) \psi_{+}, \quad \sigma \in[-1,1], \psi_{ \pm}>0, H_{1} \psi_{ \pm}=0,
$$

and $\psi_{+}$and $\psi_{-}$are linearly independent. Thus we are forced to a close examination of the zero-energy spectral properties of $H_{1}$ which will lead us to the concept of (sub)criticality of $H_{1}$. (Roughly speaking, $H_{1}$ is (sub)critical if the equation $H_{1} \psi=0$ has 1 ( 2 linearly independent) positive distributional solution(s).)

So far we have ignored the $t$-dependence of $V_{1}$ and $\phi$. Let us assume that $V_{1}$ satisfies the $\mathrm{KdV}$-equation (1.1) in what follows. Then the commutation relation for the Lax pair $\left(H_{1}(t), B_{V_{1}}(t)\right)$,

$$
\partial_{t} H_{1}-\left[B_{V_{1}}, H_{1}\right]=\operatorname{KdV}\left(V_{1}\right)
$$

where

$$
B_{V}(t)=-4 \partial_{x}^{3}+6 V(t, \cdot) \partial_{x}+3 V_{x}(t, \cdot), \quad t \in \mathbb{R},
$$

proves that $H_{1}(t)$ is unitarily equivalent to $H_{1}(0)$ for all $t \in \mathbb{R}$ and hence (1.9) holds for all $t \in \mathbb{R}$ if it holds, e.g., at $t=0$. Moreover, assuming that the $\psi_{ \pm}$ in (1.13) evolve according to $B_{V_{1}}$, i.e.,

$$
\psi_{ \pm, t}(t, x)=\left(B_{V_{1}}(t) \psi_{ \pm}(t)\right)(x), \quad(t, x) \in \mathbb{R}^{2},
$$

fixes the time-dependence of $\psi_{ \pm}$in such a way that the Wronskian of $\psi_{+}$and $\psi_{-}$becomes $t$-independent. In particular, the $\mathrm{KdV}$-flow leaves the number of linearly independent, positive solutions of $H_{1}(t) \psi(t)=0$ invariant. In other words, given $V_{1}(t, x)$ with $\operatorname{KdV}\left(V_{1}\right)=0$, we either get a unique solution $\phi_{0}(t, x)$ of (1.6) for $j=1$ or we get a one-parameter family $\phi_{\sigma}(t, x), \sigma \in$ $[-1,1]$, of such solutions. What remains to be indicated is why, in either case, $\phi_{\sigma}$ also satisfies the $\mathrm{mKdV}$-equation (1.2-) if $\sigma$ is time-independent. This 
can be made plausible by recalling that the $\mathrm{mKdV}$-equation subordinates to the AKNS-ZS framework and

$$
\begin{aligned}
& Q(t)=\left(\begin{array}{cc}
0 & A(t)^{*} \\
A(t) & 0
\end{array}\right), \quad B(t)=B_{V_{1}}(t) \oplus B_{V_{2}}(t), \\
& A(t)=\partial_{x}+\phi(t, \cdot), \quad V_{j}=\phi^{2}+(-1)^{j} \phi_{x},
\end{aligned}
$$

represent a Lax pair for the $\mathrm{mKdV}$-equation, i.e.,

$$
\partial_{t} Q-[B, Q]=\operatorname{mKdV}(\phi)\left(\begin{array}{ll}
0 & 1 \\
1 & 0
\end{array}\right) .
$$

But then the identity

$$
Q(t)^{2}=\left(\begin{array}{cc}
A(t)^{*} A(t) & 0 \\
0 & A(t) A(t)^{*}
\end{array}\right)=H_{1}(t) \oplus H_{2}(t)
$$

shows the intimate connection between the Lax pairs (1.14) and (1.18). In particular, this gives a hint as to why the parameter $\sigma$ in (1.13) should be timeindependent, since otherwise $\psi_{\sigma}$ in (1.13) would not evolve according to $B_{V_{1}}$ (see (1.16)). These statements will be justified in the first part of $\S 7$, where we shall reprove the main result of [50], viz. that the time independence of $\sigma$ in (1.13) is not only sufficient but also necessary for $\phi_{\sigma}$ to be a solution of the $\mathrm{mKdV}$-equation.

Summarizing the above, we infer that the connection between the $\mathrm{KdV}$ - and $\mathrm{mKdV}$-equations, effected by the Miura transformation

$$
\operatorname{KdV}\left(V_{j}\right)=0 \underset{V_{j}=\phi^{2}+(-1)^{j} \phi_{x}}{\stackrel{\text { Miura }}{\longleftrightarrow}} \operatorname{mKdV}(\phi)=0, \quad j=1,2,
$$

reflects itself in the connection between the two Lax pairs

$$
\left(H_{j}, B_{V_{j}}\right) \longleftrightarrow(Q, B), \quad Q^{2}=H_{1} \oplus H_{2}, B=B_{V_{1}} \oplus B_{V_{2}} .
$$

While the connection between $B_{V_{1}}, B_{V_{2}}$, and $B$ is simple (see (1.21)), the connection between the Schrödinger operators $H_{1}=A^{*} A, H_{2}=A A^{*}$ and the Dirac operator $Q=\left(\begin{array}{cc}0 & A^{*} \\ A & 0\end{array}\right)$ (being of AKNS-type) is more subtle (see $\S 2$ ).

Thus a realization of the program formulated in the paragraph following (1.4) requires the study of different topics such as commutation methods, i.e., the connections between operators of the type $A^{*} A, A A^{*}$, and $\left(\begin{array}{cc}0 & A^{*} \\ A & 0\end{array}\right)$, spectral and scattering theory for Schrödinger and Dirac operators, and inverse spectral and scattering theory for Schrödinger operators. In order to make this exposition reasonably self-contained we have included a concise treatment of these subjects in $\S \S 2-6$ : $\S 2$ provides all the general facts on commutation methods needed in $\S \S 7-10$. While the main body of this section summarizes results from various sources in the literature, Theorem 2.3(ii) (although presumably known to some experts) apparently has not appeared in print before. $\S 3$ is devoted to a detailed study of spectral and scattering properties of one-dimensional Schrödinger operators. In Theorems 3.1-3.3 we generalize known results in the case where the 
potential decays sufficiently fast as $x \rightarrow \pm \infty$ to the case where the potential has a nontrivial spatial asymptotics. This generalization is crucial in transferring soliton-like solutions of the $\mathrm{KdV}$-equation to that of the $\mathrm{mKdV}$-equation (cf. Remark 7.2(iii)). The remaining material in $\S 3$ summarizes some elements of Floquet theory for Schrödinger operators. $\S 4$, based on $\S \S 2$ and 3, carries over the results on Schrödinger operators to that of Dirac operators of the type

$$
\left(\begin{array}{cc}
m & A^{*} \\
A & -m
\end{array}\right), \quad m \in \mathbb{R}, A=\frac{d}{d x}+\phi .
$$

In particular, Theorems 4.2 and 4.3 and Lemma 4.5 appear to be new. $\S 5$ gives a short account of relative scattering to be used in $\S \S 8$ and 10 , where soliton solutions of the $(\mathrm{m}) \mathrm{KdV}$-equation relative to a trivial (i.e., constant) or periodic background solution of the $(\mathrm{m}) \mathrm{KdV}$-equation are shown to be characterized by the property of being relative reflectionless with respect to the background. In $\S 6$ we recall the process of adding eigenvalues into the spectral gaps of a background Hamiltonian since this represents a general method of constructing solitons relative to a nontrivial (not necessarily periodic) background. Theorem 6.1 , in the first part of $\S 6$, extends a known result where the background potential vanishes sufficiently fast as $x \rightarrow \pm \infty$, while the second part is devoted to a study of the corresponding periodic problem.

$\S 7$ is the main section and consists of three parts. In the first one we reprove the basic result of [50] (in fact, we provide more details than in [50]). In particular, we relate the existence of nonsingular solutions $\phi$ of the $\mathrm{mKdV}$ equation and of Miura's transformation (1.6) with $j=1$, given a $\mathrm{KdV}$-solution $V_{1}$, to the fact whether $H_{1}(0)$ in (1.5) is nonnegative or not. Moreover, recalling the notion of (sub)criticality of Schrödinger operators, we show in Theorem 7.9 that $\phi$ is uniquely determined by $V_{1}$ iff $H_{1}(0)$ is critical. The second part of $\S 7$ contains the main body of our new results. Given Theorem 7.9 we describe in detail the construction of soliton-like solutions and their basic properties in Theorem 7.14 and Remarks 7.15-7.17. The case of periodic solutions is treated in Theorem 7.18 and Remark 7.19. It turns out that, given a periodic solution $V_{1}$ of the $\mathrm{KdV}$-equation with $H_{1}(0)$ being subcritical, only the special values $\sigma= \pm 1$ in (1.13) lead to periodic solutions $\phi_{ \pm 1}(t, x)$ of the mKdV-equation. The final part of $\S 7$ contains hints at various possible extensions of our approach to a generalized $\mathrm{mKdV}$-equation of the type

$$
\phi_{t}-6\left(\phi^{2}-\Phi_{0}\right) \phi_{x}+\phi_{x x x}=0, \quad \Phi_{0} \in \mathbb{R},(t, x) \in \mathbb{R}^{2},
$$

and to the entire hierarchy of $(\mathrm{m}) \mathrm{KdV}$-equations.

In the remaining $\S \S 8-11$ we transfer particular classes of solutions of the $\mathrm{KdV}$-equation to the mKdV-equation. $\S 8$ (based on $\S \S 2-7$ ) is devoted to the transfer of the class of $\mathrm{KdV}$-soliton solutions to the mKdV-equation, thereby providing an alternative way to derive all soliton solutions of the mKdVequation. (Their original derivation in [54] uses inverse scattering techniques for Dirac systems.) In $\S 9$ (using $\S \S 2-4$ and 7) we consider the special example 
of a periodic two-zone solution $V_{1}$ of the $\mathrm{KdV}$-equation and transfer it to the $\mathrm{mKdV}$-equation. In the course of this we also extend a well-known theorem of Hochstadt's [59] in connection with periodic two-zone Schrödinger operators to the case of Dirac operators (1.22). In $\S 10$ (relying on $\S \S 2-9$ ) we first recall the construction of solitons relative to the periodic two-zone solution of the $\mathrm{KdV}$ solution $V_{1}$ considered in $\S 9$ (originally this has been done in [76]) and then transfer this solution to the $\mathrm{mKdV}$-equation. Finally, in $\S 11$, we indicate how to extend the framework of $\S 7$ to singular solutions of the $(\mathrm{m}) \mathrm{KdV}$-equation by dropping the condition of nonnegativity of $H_{1}(0)$. All mKdV-results in $\S \S 9-11$ are new. In contrast to $\S 8$ which is complete, $\S \S 9-11$ only present the simplest possible nontrivial solutions of the corresponding classes involved, e.g., a transfer of the whole class of periodic $n$-zone solutions $(n \in \mathbb{N})$ of the $\mathrm{KdV}$ equation to that of the $\mathrm{mKdV}$-equation based on hyperelliptic function theory (although particularly interesting because of links to algebraic geometry) would necessarily catapult this paper beyond any reasonable length. Thus we confined ourselves in $\S \S 9$ and 10 to the case of elliptic functions only. A similar remark applies to $\S 11$. (We note, however, that a transfer of the full class of periodic $n$ zone KdV-solutions expressed in terms of Riemann's theta function associated with the underlying hyperelliptic curve of genus $n-1$ and a transfer of a large class of singular $\mathrm{KdV}$-solutions expressed in terms of Wronski determinants has been completed in the meantime and will be published elsewhere.)

We finally remark that the methods developed in [50] and in the present paper are by no means restricted to the $\mathrm{KdV}$ - and $\mathrm{mKdV}$-equations. In fact, they also apply in a $1+2$-dimensional context such as the Kadomtsev-Petviashviliequation and its modified analog as well as in the context of discrete nonlinear systems such as the Toda lattice and its modified version the Kac-van Moerbeke lattice (corresponding manuscripts are in preparation).

\section{BASIC FACTS ON COMMUTATION}

In this section we review certain formulas based on commutation and add a few new results of this type in Theorem 2.3(ii).

We introduce the following hypothesis:

Let $\mathfrak{H}_{j}, j=1,2$, be separable, complex Hilbert spaces and $A$ :

$\mathfrak{D}(A) \subseteq \mathfrak{H}_{1} \rightarrow \mathfrak{H}_{2}$ a densely defined, closed linear operator.

We are particularly concerned with abstract Dirac operators in $\mathfrak{H}_{1} \oplus \mathfrak{H}_{2}$ of the type

$$
Q:=\left(\begin{array}{cc}
0 & A^{*} \\
A & 0
\end{array}\right), \quad \mathfrak{D}(Q)=\mathfrak{D}(A) \oplus \mathfrak{D}\left(A^{*}\right),
$$

and

$$
\begin{gathered}
Q_{m_{1}, m_{2}}:=\left(\begin{array}{cc}
m_{1} 1 & A^{*} \\
A & -m_{2} 1
\end{array}\right), \\
\mathfrak{D}\left(Q_{m_{1}, m_{2}}\right)=\mathfrak{D}(Q), \quad m_{j} \in \mathbb{R}, j=1,2 .
\end{gathered}
$$


Because of the identity

$$
Q_{m_{1}, m_{2}}=\left[\left(m_{1}-m_{2}\right) / 2\right] 1+Q_{\left(m_{1}+m_{2}\right) / 2},
$$

where

$$
Q_{m}:=\left(\begin{array}{cc}
m 1 & A^{*} \\
A & -m 1
\end{array}\right), \quad m \in \mathbb{R},
$$

we shall only consider the symmetric version (2.4). We note that

$$
Q_{m}^{2}=\left(\begin{array}{cc}
H_{1, m} & 0 \\
0 & H_{2, m}
\end{array}\right)=Q^{2}+m^{2} 1, \quad m \in \mathbb{R},
$$

where

$$
\begin{aligned}
H_{1, m} & :=A^{*} A+m^{2} \quad \text { on } \mathfrak{D}\left(A^{*} A\right) \subseteq \mathfrak{H}_{1}, \\
H_{2, m} & :=A A^{*}+m^{2} \quad \text { on } \mathfrak{D}\left(A A^{*}\right) \subseteq \mathfrak{H}_{2}, \quad m \in \mathbb{R}, \\
H_{1,0} & :=H_{1}=A^{*} A, \quad H_{2,0}:=H_{2}=A A^{*}
\end{aligned}
$$

(we delete the symbol 1 in multiples of the identity operator in $\mathfrak{H}_{j}, j=1,2$, and $\mathfrak{H}_{1} \oplus \mathfrak{H}_{2}$ from now on).

Next we collect a few useful commutation formulas.

Theorem 2.1 [32]. Assume hypothesis (H.2.1) and let $f \in L^{\infty}(\mathbb{R})$. Then

$$
A=S H_{1}^{1 / 2}=H_{2}^{1 / 2} S,
$$

where $S:=\operatorname{sgn}(A)$ denotes the partial isometry with initial set $\overline{\operatorname{Ran}\left[\left(A^{*} A\right)^{1 / 2}\right]}$ and final set $\overline{\operatorname{Ran}(A)}$ in the polar decomposition of $A$.

(ii)

$$
\operatorname{sgn}(Q)=\left(\begin{array}{cc}
0 & S^{*} \\
S & 0
\end{array}\right)
$$

is the corresponding partial isometry in the polar decomposition of $Q$.

$$
\begin{aligned}
& \operatorname{Ker}(A)=\operatorname{Ker}\left(H_{1}\right)=\operatorname{Ran}\left(A^{*}\right)^{\perp}, \\
& \operatorname{Ker}\left(A^{*}\right)=\operatorname{Ker}\left(H_{2}\right)=\operatorname{Ran}(A)^{\perp} .
\end{aligned}
$$

$$
\begin{gathered}
S H_{1}^{n / 2} \subseteq H_{2}^{n / 2} S, \quad n \in \mathbb{N}, \\
S f\left(H_{1}\right)=f\left(H_{2}\right) S .
\end{gathered}
$$

In particular, $H_{1}$ and $H_{2}$ are essentially isospectral, i.e.,

$$
\sigma\left(H_{1}\right) \backslash\{0\}=\sigma\left(H_{2}\right) \backslash\{0\}
$$


and

$$
\begin{array}{cl}
H_{1} \psi=E \psi, & E \neq 0 \Rightarrow H_{2}(A \psi)=E(A \psi), \quad \psi \in \mathfrak{D}\left(H_{1}\right), \\
H_{2} \tilde{\psi}=\widetilde{E} \tilde{\psi}, & \widetilde{E} \neq 0 \Rightarrow H_{1}\left(A^{*} \tilde{\psi}\right)=\widetilde{E}\left(A^{*} \tilde{\psi}\right), \quad \tilde{\psi} \in \mathfrak{D}\left(H_{2}\right),
\end{array}
$$

with multiplicities preserved.

(v)

$$
\begin{aligned}
& 1+z\left(H_{2}-z\right)^{-1} \supseteq A\left(H_{1}-z\right)^{-1} A^{*}, \\
& 1+z\left(H_{1}-z\right)^{-1} \supseteq A^{*}\left(H_{2}-z\right)^{-1} A, \quad z \in \mathbb{C} \backslash\left\{\sigma\left(H_{1}\right) \cup \sigma\left(H_{2}\right)\right\} .
\end{aligned}
$$

$$
A^{*} f\left(H_{2}\right) \supseteq f\left(H_{1}\right) A^{*}, \quad A f\left(H_{1}\right) \supseteq f\left(H_{2}\right) A .
$$

Sketch of proof. Let $T$ denote a densely defined, closed linear operator from $\mathfrak{D}(T) \subseteq \mathfrak{H}^{\prime} \rightarrow \mathfrak{H}^{\prime \prime}$, where $\mathfrak{H}^{\prime}, \mathfrak{H}^{\prime \prime}$ are separable, complex Hilbert spaces. Then $\operatorname{sgn}(T)$ is defined by

$$
\operatorname{sgn}(T):= \begin{cases}\overline{T\left(T^{*} T\right)^{-1 / 2}} \text { on } \operatorname{Ker}(T)^{\perp}, \\ 0 & \text { on } \operatorname{Ker}(T)\end{cases}
$$

The spectral theorem for selfadjoint operators together with the following obvious equalities then proves (i)-(vi):

$$
\begin{gathered}
Q=|Q| \operatorname{sgn}(Q)=\operatorname{sgn}(Q)|Q|, \\
\operatorname{Ker}(Q)=\operatorname{Ker}\left(Q^{2}\right)=\operatorname{Ran}(Q)^{\perp}, \\
1+z\left(Q^{2}-z\right)^{-1}=Q^{2}\left(Q^{2}-z\right)^{-1} \supseteq Q\left(Q^{2}-z\right)^{-1} Q, \quad z \in \mathbb{C} \backslash \sigma\left(Q^{2}\right), \\
Q f\left(Q^{2}\right) \supseteq f\left(Q^{2}\right) Q .
\end{gathered}
$$

Remark 2.2. Theorem 2.1 is due to Deift [32]. The idea of proof, presented above, is due to Nelson (unpublished) and has been summarized in [109].

Theorem 2.3. Assume (H.2.1). Then

(i)

$$
\begin{array}{cc}
\left(Q_{m}-z\right)^{-1}=\left(\begin{array}{cc}
\left(H_{1, m}-z^{2}\right)^{-1}(z+m) & A^{*}\left(H_{2, m}-z^{2}\right)^{-1} \\
A\left(H_{1, m}-z^{2}\right)^{-1} & \left(H_{2, m}-z^{2}\right)^{-1}(z-m)
\end{array}\right), \\
& z^{2} \in \mathbb{C} \backslash\left\{\sigma\left(H_{1, m}\right) \cup \sigma\left(H_{2, m}\right)\right\}
\end{array}
$$

(ii) Define

$$
R:=i \sigma_{3} \operatorname{sgn}(Q)=\left(\begin{array}{cc}
0 & i S^{*} \\
-i S & 0
\end{array}\right), \quad \sigma_{3}=\left(\begin{array}{cc}
1 & 0 \\
0 & -1
\end{array}\right)
$$

then

$$
\begin{gathered}
\operatorname{Ker}(R)=\operatorname{Ker}(Q)=\operatorname{Ker}(A) \oplus \operatorname{Ker}\left(A^{*}\right), \\
R^{2}=\left(\begin{array}{cc}
S^{*} S & 0 \\
0 & S S^{*}
\end{array}\right), \quad Q_{m} R=-R Q_{m}, \quad m \in \mathbb{R}
\end{gathered}
$$


(together with $A=S|A|=\left|A^{*}\right| S, A^{*}=S^{*}\left|A^{*}\right|=|A| S^{*}$ ), shows that $Q_{m}$ is unitarily equivalent to $-Q_{m}$ on the subspace $\operatorname{Ker}(Q)^{\perp}$ of $\mathfrak{H}_{1} \oplus \mathfrak{H}_{2}$, i.e.,

$$
Q_{m}=-R Q_{m} R \text { on } \operatorname{Ker}(Q)^{\perp}, m \in \mathbb{R} \text {. }
$$

Moreover,

$$
Q_{m}^{2} \geq m^{2}, \quad m \in \mathbb{R},
$$

and $Q$ is unitarily equivalent to $-Q$, i.e.,

$$
\sigma_{3} Q \sigma_{3}=-Q \text {. }
$$

Proof. (i) is most easily verified by multiplying with $\left(Q_{m}-z\right)$ from the left and right respectively. (ii) is obvious from the fact that $S^{*} S$ is the projection onto $\operatorname{Ker}(A)$ and $S S^{*}$ is the projection onto $\operatorname{Ker}\left(A^{*}\right)$.

Remark 2.4. (i) By identity (2.3), these results extend to $Q_{m_{1}, m_{2}}$ in a straightforward manner.

(ii) Since by (2.22),

$$
Q_{m}^{2}=R Q_{m}^{2} R \text { on } \operatorname{Ker}(Q)^{\perp}, m \in \mathbb{R},
$$

we infer that $\left.A^{*} A\right|_{\operatorname{Ker}(A)^{\perp}}$ and $\left.A A^{*}\right|_{\operatorname{Ker}\left(A^{*}\right)^{\perp}}$ are unitarily equivalent, i.e.,

$$
\begin{array}{ll}
A^{*} A=S^{*} A A^{*} S & \text { on } \operatorname{Ker}(A)^{\perp}, \\
A A^{*}=S A^{*} A S^{*} & \text { on } \operatorname{Ker}\left(A^{*}\right)^{\perp} .
\end{array}
$$

Thus we recover Theorem 3 of [32].

The above assertions may be supplemented by the following results.

Theorem 2.5 [109]. Assume (H.2.1).

(i) There exists a unitary operator $U_{m}$ on $\operatorname{Ker}(Q)^{\perp}$ such that

$$
U_{m} Q_{m} U_{m}^{*}=\left(\begin{array}{cc}
H_{1, m}^{1 / 2} & 0 \\
0 & -H_{2, m}^{1 / 2}
\end{array}\right) \quad \text { on } \operatorname{Ker}(Q)^{\perp}
$$

$$
U_{m}=\left(\begin{array}{cc}
a_{1,+} & a_{1,-} S^{*} \\
-a_{2,-} S & a_{2,+}
\end{array}\right),
$$

$a_{j, \pm}:=2^{-1 / 2}\left\{1 \pm m\left(H_{j}+m^{2}\right)^{-1 / 2}\right\}^{1 / 2}, j=1,2, m \in \mathbb{R}$.

(ii) There exists a unitary operator $W_{m}$ on $\operatorname{Ker}(Q)^{\perp}$ such that

$$
\begin{gathered}
W_{m} Q_{m} W_{m}^{*}=\left|Q_{m}\right| \operatorname{sgn}(Q)=\left(\begin{array}{cc}
0 & H_{1, m}^{1 / 2} S^{*} \\
H_{2, m}^{1 / 2} S & 0
\end{array}\right) \text { on } \operatorname{Ker}(Q)^{\perp}, \\
W_{m}:=2^{-1 / 2}\left(\begin{array}{cc}
1 & -S^{*} \\
S & 1
\end{array}\right) U_{m}, \quad m \in \mathbb{R} .
\end{gathered}
$$




\section{ONE-DIMENSIONAL SCHRÖDINGER OPERATORS}

In this section we study Schrödinger operators $H_{1}=A^{*} A, H_{2}=A A^{*}$ obtained from a concrete realization of $A$ in $L^{2}(\mathbb{R})$, formally given by $\frac{d}{d x}+\phi(x)$, where $\phi$ satisfies one of the following hypotheses:

(H.3.1) $\phi, \phi^{\prime} \in L^{\infty}(\mathbb{R})$ real-valued,

$$
\begin{array}{cc}
\lim _{x \rightarrow \pm \infty} \phi(x):=\phi_{ \pm} \in \mathbb{R}, & \phi_{-}^{2} \leq \phi_{+}^{2}, \\
\pm \int_{0}^{ \pm \infty} d x\left(1+x^{2}\right)\left|\phi(x)-\phi_{ \pm}\right|<\infty, & \int_{\mathbb{R}} d x\left(1+x^{2}\right)\left|\phi^{\prime}(x)\right|<\infty .
\end{array}
$$
$\mathbb{R}$.

(H.3.2) $\phi, \phi^{\prime} \in L^{\infty}(\mathbb{R})$ real-valued, for some $a>0: \phi(x+a)=\phi(x), x \in$

The factorization of 2nd-order Sturm-Liouville operators has a long history: It dates back at least to Jacobi [63], respectively Darboux [29]. Other important contributions are $[19,20,28,99]$ (see also the references therein) and especially [32]. Quite recently these ideas became popular again in connection with supersymmetric quantum mechanics $[8,16,17,24,49,64,91,104,109]$ (see also the references cited therein).

In both cases (H.3.1) and (H.3.2) $A$ is defined in $L^{2}(\mathbb{R})$ by

$$
A=\frac{d}{d x}+\phi, \quad \mathfrak{D}(A)=H^{1}(\mathbb{R}),
$$

and hence

$$
\begin{aligned}
& H_{j}=-\frac{d^{2}}{d x^{2}}+\phi^{2}+(-1)^{j} \phi^{\prime}:=-\frac{d^{2}}{d x^{2}}+V_{j}, \\
& \mathfrak{D}\left(H_{j}\right)=H^{2}(\mathbb{R}), \quad j=1,2 .
\end{aligned}
$$

Next we briefly summarize spectral and scattering theory for systems governed by (H.3.1). (For more details see, e.g., [16, 23, 27, 31, 33, 49, 96].) Let $f_{j, \pm}\left(k_{ \pm}, x\right)$, defined by

$$
\begin{aligned}
& f_{j, \pm}\left(k_{ \pm}, x\right) \\
& \quad=e^{ \pm i k_{ \pm} x}-\int_{x}^{ \pm \infty} d x^{\prime} k_{ \pm}^{-1} \sin \left[k_{ \pm}\left(x-x^{\prime}\right)\right]\left[V_{j}\left(x^{\prime}\right)-V_{ \pm}\right] f_{j, \pm}\left(k_{ \pm}, x^{\prime}\right),
\end{aligned}
$$

$$
k_{ \pm}:=\left(z-V_{ \pm}\right)^{1 / 2}, \quad \operatorname{Im} k_{ \pm} \geq 0, \quad z \in \mathbb{C}, \quad V_{ \pm}:=\phi_{ \pm}^{2}, \quad j=1,2,
$$

be the Jost solutions of $H_{j}$, i.e.,

$$
H_{j} f_{j, \pm}\left(k_{ \pm}, x\right)=z f_{j, \pm}\left(k_{ \pm}, x\right), \quad z \in \mathbb{C}, j=1,2,
$$

in the distributional sense. Clearly

$$
W\left(f_{j, \mp}\left(-k_{\mp}\right), f_{j, \mp}\left(k_{\mp}\right)\right)=\mp 2 i k_{\mp}, \quad z>V_{\mp}, j=1,2,
$$

where $W(F, G)(x):=F(x) G^{\prime}(x)-F^{\prime}(x) G(x)$ denotes the Wronskian of $F$ and $G$. Spectral properties of $H_{j}$ are reviewed in 
Theorem 3.1. Assume (H.3.1). Then

$$
\sigma_{\text {ess }}\left(H_{j}\right)=\sigma_{\mathrm{ac}}\left(H_{j}\right)=\left[\phi_{-}^{2}, \infty\right), \quad \sigma_{\mathrm{sc}}\left(H_{j}\right)=\varnothing, j=1,2 .
$$

Moreover, the $H_{j}, j=1,2$, have simple spectrum in $\left(\phi_{-}^{2}, \phi_{+}^{2}\right)$ (iff $\phi_{-}^{2}<\phi_{+}^{2}$, otherwise delete this assertion) and spectral multiplicity two in $\left(\phi_{+}^{2}, \infty\right)$. In addition, the $H_{j}, j=1,2$, have finitely many simple eigenvalues $\lambda_{j, l}=\phi_{ \pm}^{2}-$ $\kappa_{j, \pm, l}^{2}$ in $\left[0, \phi_{-}^{2}\right)$ determined by

$$
W\left(f_{j,-}\left(i \kappa_{j,-, l}\right), f_{j,+}\left(i \kappa_{j,+, l}\right)\right)=0, \quad j=1,2 .
$$

If $\phi_{-}^{2}>0$, the (simple) eigenvalues of $H_{1}$ and $H_{2}$ coincide in $\left(0, \phi_{-}^{2}\right)$. There are no eigenvalues embedded into the essential spectrum and there are no threshold eigenvalues, i.e.,

$$
\sigma_{p}\left(H_{j}\right) \cap\left[\phi_{-}^{2}, \infty\right)=\varnothing, \quad j=1,2 .
$$

If $W\left(f_{j,-}(0), f_{j,+}\left(i\left(\phi_{+}^{2}-\phi_{-}^{2}\right)^{1 / 2}\right)\right)=0$, then $H_{j}$ has a threshold resonance whose wave function $\psi_{j}$ (suitably normalized) satisfies

$$
\begin{aligned}
& \left|\psi_{j}(x)-1\right| \leq c(1+|x|)^{-1}, \quad x \leq 0, \\
& \left|\psi_{j}(x)\right| \leq c^{\prime} e^{-\left(\phi_{+}^{2}-\phi_{-}^{2}\right)^{1 / 2} x}, \quad x \geq 0, j=1,2 .
\end{aligned}
$$

Next we describe the unitary on-shell scattering matrix $S_{j}(\lambda)$ associated with $H_{j}$.

Theorem 3.2. Assume (H.3.1). Then if

(i) $\lambda>\phi_{+}^{2}: S_{j}(\lambda)$ in $\mathbb{C}^{2}$ is given by

$$
S_{j}(\lambda)=\left(\begin{array}{cc}
T_{j}(\lambda) & R_{j}^{r}(\lambda) \\
R_{j}^{l}(\lambda) & T_{j}(\lambda)
\end{array}\right), \quad j=1,2,
$$

where the transmission and reflection coefficients from the left and right are given by

$$
\begin{aligned}
T_{j}(\lambda) & =2 i\left(k_{+} k_{-}\right)^{1 / 2} / W\left(f_{j,-}\left(k_{-}\right), f_{j,+}\left(k_{+}\right)\right), \\
R_{j}^{l}(\lambda) & =-W\left(f_{j,-}\left(-k_{-}\right), f_{j,+}\left(k_{+}\right)\right) / W\left(f_{j,-}\left(k_{-}\right), f_{j,+}\left(k_{+}\right)\right), \\
R_{j}^{r}(\lambda) & =-W\left(f_{j,-}\left(k_{-}\right), f_{j,+}\left(-k_{+}\right)\right) / W\left(f_{j,-}\left(k_{-}\right), f_{j,+}\left(k_{+}\right)\right), \\
& k_{ \pm}=\left(\lambda-\phi_{ \pm}^{2}\right)^{1 / 2}>0, \lambda>\phi_{+}^{2}, j=1,2 .
\end{aligned}
$$

(ii) $\phi_{-}^{2}<\lambda<\phi_{+}^{2}$ :

$$
S_{j}(\lambda)=-\overline{W\left(f_{j,-}\left(k_{-}\right), f_{j,+}\left(k_{+}\right)\right)} / W\left(f_{j,-}\left(k_{-}\right), f_{j,+}\left(k_{+}\right)\right), \quad l .
$$


Unitarity of $S_{j}(\lambda)$ now trivially follows from (3.6) and the identity

$$
\begin{aligned}
W(F, G)(x) W(f, g)(x)= & W(F, g)(x) W(f, G)(x) \\
& -W(F, f)(x) W(g, G)(x) .
\end{aligned}
$$

In particular,

$$
\begin{aligned}
& \left|T_{j}(\lambda)\right|^{2}+\left|R_{j}^{l}(\lambda)\right|^{2}=1=\left|T_{j}(\lambda)\right|^{2}+\left|R_{j}^{r}(\lambda)\right|^{2}, \\
& \left|R_{j}^{l}(\lambda)\right|=\left|R_{j}^{r}(\lambda)\right|, \quad \lambda>\phi_{+}^{2}, \quad j=1,2 .
\end{aligned}
$$

So far we have treated $S_{1}(\lambda)$ and $S_{2}(\lambda)$ separately. However, $\S 2$ suggests that actually there should be a close connection between them. First of all we infer that

$$
\left\{\begin{array}{l}
f_{1, \pm}\left(k_{ \pm}, x\right), \\
f_{2, \pm}\left(k_{ \pm}, x\right)=\left( \pm i k_{ \pm}+\phi_{ \pm}\right)^{-1}\left(A f_{1, \pm}\right)\left(k_{ \pm}, x\right)
\end{array}\right.
$$

are correctly normalized Jost solutions of $H_{1}$ and $H_{2}$. Using the elementary identity

$$
W(A f(z), A g(z))=z W(f(z), g(z)), \quad z \in \mathbb{C},
$$

where $f(z, x), g(z, x)$ are any distributional solutions of

$$
\left(A^{*} A \psi(z)\right)(x)=\left(H_{1} \psi(z)\right)(x)=z \psi(z, x), \quad z \in \mathbb{C},
$$

we obtain

Theorem 3.3. Assume (H.3.1). Then if

(i) $\lambda>\phi_{+}^{2}$ :

$$
\begin{aligned}
& T_{1}(\lambda)=\left(i k_{-}+\phi_{-}\right)\left(i k_{+}+\phi_{+}\right)^{-1} T_{2}(\lambda), \\
& R_{1}^{l}(\lambda)=\left(i k_{-}+\phi_{-}\right)\left(-i k_{-}+\phi_{-}\right)^{-1} R_{2}^{l}(\lambda), \\
& R_{1}^{r}(\lambda)=\left(-i k_{+}+\phi_{+}\right)\left(i k_{+}+\phi_{+}\right)^{-1} R_{2}^{r}(\lambda) .
\end{aligned}
$$

(ii) $\phi_{-}^{2}<\lambda<\phi_{+}^{2}$ :

$$
S_{1}(\lambda)=\left(i k_{-}+\phi_{-}\right)\left(-i k_{-}+\phi_{-}\right)^{-1} S_{2}(\lambda) .
$$

Remark 3.4. We also note that the norming constants $c_{j, \pm, l}$, associated with nonzero eigenvalues $\lambda_{j, l}=\phi_{ \pm}^{2}-\kappa_{j, \pm, l}^{2}$ of $H_{j}, j=1,2$, are connected with each other as follows. Let

$$
\left|c_{j, \pm l}\right|:=\left\|f_{j, \pm, l}\right\|_{2}^{-1}, \quad j=1,2, l=1,2, \ldots,
$$

where

$$
\begin{aligned}
& H_{j} f_{j, \pm, l}=\lambda_{j, l} f_{j, \pm, l}, \quad f_{j, \pm, l} \in H^{2}(\mathbb{R}), \\
& f_{j, \pm, l}\left(i \kappa_{j, \pm, l}, x\right) \underset{x \rightarrow \pm \infty}{=} e^{\mp \kappa_{j, \pm, l} x}+o\left(e^{\mp \kappa_{j, \pm} l^{x}}\right) \text {, } \\
& 0<\kappa_{j, \pm, l} \leq\left|\phi_{ \pm}\right|, j=1,2, \\
& f_{1, \pm, l}\left(i \kappa_{1, \pm, l}, x\right)=\left(\phi_{ \pm} \pm \kappa_{1, \pm, l}\right)^{-1}\left(A^{*} f_{2, \pm, l}\right)\left(i \kappa_{1, \pm, l}, x\right) \text {, } \\
& 0<\kappa_{1, \pm, l}<\left|\phi_{ \pm}\right|, l=1,2, \ldots \text {. }
\end{aligned}
$$


Then

(3.23)

$$
\begin{aligned}
\left|c_{1, \pm, l}\right| & =\left\|f_{1, \pm, l}\right\|_{2}^{-1}=\left(\phi_{ \pm} \pm \kappa_{1, \pm, l}\right)\left\|A^{*} f_{2, \pm, l}\right\|_{2}^{-1} \\
& =\left[\left(\phi_{ \pm} \pm \kappa_{1, \pm, l}\right)\left(\phi_{ \pm} \mp \kappa_{1, \pm, l}\right)^{-1}\right]^{1 / 2}\left|c_{2, \pm, l}\right|, \quad l=1,2, \ldots .
\end{aligned}
$$

Remark 3.5. As discussed in [49], the hypothesis (H.3.1) in Theorems 3.1-3.3 can be considerably weakened in the sense that for most of the results stated above one only needs the zeroth or first moments of $\left|\phi(x)-\phi_{ \pm}\right|,\left|\phi^{\prime}(x)\right|$ to be finite.

Now we briefly turn to systems governed by (H.3.2). Since now $V_{j}(x)=$ $\phi^{2}(x)+(-1)^{j} \phi^{\prime}(x)$ are periodic, Floquet theory (see, e.g. [30, 38, 62, 79, 85, $86,95,110,112])$ applies. To fix the notation, we briefly introduce normalized Floquet solutions [43, 45]:

$$
\begin{aligned}
& f_{j, \pm}(k, x):=\psi_{j, \pm}(k, x) a^{1 / 2} /\left\|\psi_{j}(k)\right\|_{2}, \\
& \left\|\psi_{j}(k)\right\|_{2}^{2}:=\int_{0}^{a} d x \psi_{j,+}(k, x) \psi_{j,-}(k, x),
\end{aligned}
$$

where the Floquet solutions $\psi_{j, \pm}(k, x)$ are defined as follows. Let

$$
\begin{aligned}
& \left(H_{j} \theta_{j}(z)\right)(x)=z \theta_{j}(z, x), \\
& \left(H_{j} \varphi_{j}(z)\right)(x)=z \varphi_{j}(z, x), \quad z \in \mathbb{C}, j=1,2,
\end{aligned}
$$

in the distributional sense with

$$
\begin{aligned}
& \theta_{j}(z, 0)=1, \quad \theta_{j}^{\prime}(z, 0)=0, \\
& \varphi_{j}(z, 0)=0, \quad \varphi_{j}^{\prime}(z, 0)=1, \quad z \in \mathbb{C}, j=1,2,
\end{aligned}
$$

and define the discriminant $F_{j}$ by

$$
F_{j}(z):=\left[\theta_{j}(z, a)+\varphi_{j}^{\prime}(z, a)\right] / 2, \quad z \in \mathbb{C}, j=1,2 .
$$

By taking into account that $H_{1}$ and $H_{2}$ are essentially isospectral, one derives that

$$
\begin{aligned}
& \theta_{1}(z, x), \varphi_{1}(z, x), \\
& \theta_{2}(z, x)=A\left\{z^{-1} \phi(0) \theta_{1}(z, x)+\left[1-z^{-1} \phi(0)^{2}\right] \varphi_{1}(z, x)\right\}, \\
& \varphi_{2}(z, x)=A\left\{-z^{-1} \theta_{1}(z, x)+z^{-1} \phi(0) \varphi_{1}(z, x)\right\}, \quad z \in \mathbb{C} \backslash\{0\},
\end{aligned}
$$

satisfy the boundary conditions (3.27). Thus we actually infer

$$
F_{1}(z)=F_{2}(z):=F(z), \quad z \in \mathbb{C} .
$$

Now the $\psi_{j, \pm}$ are defined by

$$
\psi_{j, \pm}(k, x):=\theta_{j}(z(k), x)+m_{j, \pm}(z(k)) \varphi_{j}(z(k), x), \quad j=1,2,
$$

where

$$
m_{j, \pm}(z):=\left\{\varphi_{j}^{\prime}(z, a)-\theta_{j}(z, a) \mp 2 \sqrt{F(z)^{2}-1}\right\} / 2 \varphi_{j}(z, a),
$$


$z \in \mathbb{C} \backslash\left\{\lambda_{n} \in \mathbb{R} \mid n \in \mathbb{N}\right\}, \quad \sqrt{F(\lambda)^{2}-1}>0$ for $\lambda<\inf \left[\sigma\left(H_{j}\right)\right], j=1,2$, with $\lambda_{n}, n \in \mathbb{N}$, given by

$$
F_{z}\left(\lambda_{n}\right)=0, \quad n \in \mathbb{N},
$$

and the Floquet parameter $k$ defined by

$$
k(z):=\arcsin \left[i \sqrt{F(z)^{2}-1}\right] / a, \quad z \in \mathbb{C} .
$$

In addition one has

$$
\begin{gathered}
f_{j, \pm}(k, x)=e^{ \pm i k x} p_{j, \pm}(k, x), \quad p_{j, \pm}(k, x+a)=p_{j, \pm}(k, x), \\
j=1,2, \\
f_{j, \pm}(-k, x)=f_{j, \mp}(k, x)=\overline{f_{j, \pm}(k, x)}, \quad k \in \mathbb{R}, j=1,2, \\
F_{z}(z)=-\varphi_{j}(z, a)\left\|\psi_{j}(k(z))\right\|_{2}^{2} / 2, \quad z \in \mathbb{C}, j=1,2, \\
\begin{aligned}
& W\left(f_{j, \mp}(-k), f_{j, \mp}(k)\right)= \pm a i \sin (k a) / F_{z}(z(k)) \\
&= \mp a \sqrt{F(z(k))^{2}-1} / F_{z}(z(k)), \\
& z \in \mathbb{C} \backslash\left\{\lambda_{n} \mid n \in \mathbb{N}\right\}, j=1,2 .
\end{aligned}
\end{gathered}
$$

Theorem 3.6. Assume (H.3.2). Then the spectra of $H_{1}$ and $H_{2}$ coincide. They are purely absolutely continuous and of multiplicity two. Moreover,

$$
\begin{aligned}
& \sigma\left(H_{j}\right)=\sigma_{\mathrm{ac}}\left(H_{j}\right)=\{\lambda \in \mathbb{R}|| F(\lambda) \mid \leq 1\}=\bigcup_{n \in N} \sigma_{n}, \\
& \sigma_{n}:=\left[E_{2(n-1)}, E_{2 n-1}\right], \quad n \in \mathbb{N}, \\
& 0 \leq E_{0}<E_{1} \leq E_{2}<E_{3} \leq E_{4}<\cdots, \\
& \sigma_{p}\left(H_{j}\right)=\sigma_{\mathrm{sc}}\left(H_{j}\right)=\varnothing, \quad j=1,2 .
\end{aligned}
$$

Remark 3.7. (i) The fact that $F_{1}(z)=F_{2}(z)$ is noi surprising when taking into account that $[95$, p. 384]

$$
\frac{F_{j}(z)-2}{F_{0}\left(z^{\prime}\right)-2}=\operatorname{det}\left[\left(h_{0}-z^{\prime}\right)^{-1}\left(h_{j}-z\right)\right]
$$

$z \in \mathbb{C}, z^{\prime} \in \mathbb{C} \backslash \sigma\left(h_{0}\right), \quad j=1,2$,

where

$$
\begin{aligned}
& h_{0}=-\frac{d^{2}}{d x^{2}}, \quad \mathfrak{D}\left(h_{0}\right)=\left\{g \in H^{2}((0, a)) \mid g\left(0_{+}\right)=g\left(a_{-}\right), g^{\prime}\left(0_{+}\right)=g^{\prime}\left(a_{-}\right)\right\}, \\
& h_{j}=-\frac{d^{2}}{d x^{2}}+V_{j}, \quad \mathfrak{D}\left(h_{j}\right)=\mathfrak{D}\left(h_{0}\right), \quad j=1,2,
\end{aligned}
$$


are the reduced operators in $L^{2}((0, a))$ with periodic boundary conditions,

$$
F_{0}(z):=2 \cos \left(z^{1 / 2}\right), \quad z \in \mathbb{C},
$$

the corresponding unperturbed discriminant, and det (.) denotes the Fredholm determinant. Thus

$$
\frac{F_{1}(z)-2}{F_{2}(z)-2}=\operatorname{det}\left[\left(h_{2}-z\right)^{-1}\left(h_{1}-z\right)\right]=1, \quad z \in \mathbb{C} \backslash \sigma\left(h_{2}\right),
$$

by applying commutation to $h_{1}$ and $h_{2}$. More generally, applying the Hadamard factorization theorem to $F_{1}(\cdot)$, one can prove that $F_{1}(\cdot)$ is an invariant for all periodic potentials (with period $a>0$ ) in the isospectral manifold of $V_{1}$ (see, e.g., [21]).

(ii) Not only the effective masses (i.e., $d^{2} k /\left.d \lambda^{2}\right|_{\lambda=E_{n}}, n \in \mathbb{N}$ ) of $V_{1}$ and $V_{2}$ coincide (cf. [103]) but also the Floquet parameters and the dispersion laws associated with $H_{1}$ and $H_{2}$ are identical in spite of the fact that $V_{1} \neq V_{2}$ (excluding the trivial case $\phi=$ const).

For further applications of commutation in the periodic case see $\S 9$.

We end this section with a simple observation:

Remark 3.8. Let $\phi, \phi^{\prime} \in L^{\infty}(\mathbb{R})$ be real-valued, $A=\frac{d}{d x}+\phi$ on $H^{1}(\mathbb{R})$. Then $H_{1}=A^{*} A$ and $H_{2}=A A^{*}$ cannot simultaneously have zero as an eigenvalue. Indeed, if

$$
H_{1} \psi_{1}=0, \quad \psi_{1} \in H^{2}(\mathbb{R}), \quad \psi_{1}(x)=C \exp \left[-\int_{0}^{x} d x^{\prime} \phi\left(x^{\prime}\right)\right],
$$

then

$$
H_{2}\left(\psi_{1}^{-1}\right)=0
$$

in the distributional sense and the assumption $\psi_{1}^{-1} \in L^{2}(\mathbb{R})$ (together with the fact that 0 must be a nondegenerate eigenvalue of $H_{1}$ ) leads to the contradiction

$$
\propto=\int_{\mathbb{R}} d x \psi_{1}(x) \psi_{1}(x)^{-1} \leq\left\|\psi_{1}\right\|_{2}\left\|\psi_{1}^{-1}\right\|_{2}<\infty .
$$

\section{ONE-Dimensional DiRac OpERATORS}

Given the explicit realizations of $A$ in $L^{2}(\mathbb{R})$ of the foregoing section, we now study the associated Dirac operator

$$
Q_{m}=\left(\begin{array}{cc}
m & A^{*} \\
A & -m
\end{array}\right), \quad m \in \mathbb{R},
$$

in $L^{2}(\mathbb{R}) \otimes \mathbb{C}^{2}$.

Since Theorems 2.3 and 2.5 directly apply to (4.1), we do not need to repeat them here.

First we consider systems satisfying (H.3.1). Combining Theorem 3.1 and the results of $\S 2$ then yields the following theorem. 
Theorem 4.1. Assume (H.3.1). Then

$$
\begin{aligned}
& \sigma_{\mathrm{ess}}\left(Q_{m}\right)=\sigma_{\mathrm{ac}}\left(Q_{m}\right)=\left(-\infty,-\left(\phi_{-}^{2}+m^{2}\right)^{1 / 2}\right] \cup\left[\left(\phi_{-}^{2}+m^{2}\right)^{1 / 2}, \infty\right), \\
& \sigma_{\mathrm{sc}}\left(Q_{m}\right)=\varnothing, \quad m \in \mathbb{R},
\end{aligned}
$$

and $Q_{m}$ has spectral multiplicity one in $\left(-\left(\phi_{+}^{2}+m^{2}\right)^{1 / 2},-\left(\phi_{-}^{2}+m^{2}\right)^{1 / 2}\right) \cup$ $\left(\left(\phi_{-}^{2}+m^{2}\right)^{1 / 2},\left(\phi_{+}^{2}+m^{2}\right)^{1 / 2}\right)$ (iff $\phi_{-}^{2}<\phi_{+}^{2}$, otherwise delete this assertion) and spectral multiplicity two in $\left(-\infty,-\left(\phi_{+}^{2}+m^{2}\right)^{1 / 2}\right) \cup\left(\left(\phi_{+}^{2}+m^{2}\right)^{1 / 2}, \infty\right)$. In addition $Q_{m}$ has finitely many simple eigenvalues in $\left(-\left(\phi_{-}^{2}+m^{2}\right)^{1 / 2}\right.$, $\left(\phi_{-}^{2}+m^{2}\right)^{1 / 2}$ ) (assuming $\phi_{-} \neq 0$ or $m \neq 0$ ) symmetrically placed w.r.t. zero with the possible exception at $\pm m$ (if both $\phi_{-} \neq 0, m \neq 0$ ). There are no eigenvalues embedded in the essential spectrum and there are no threshold bound states, i.e.,

$$
\sigma_{p}\left(Q_{m}\right) \cap\left\{\left(-\infty,-\left(\phi_{-}^{2}+m^{2}\right)^{1 / 2}\right] \cup\left[\left(\phi_{-}^{2}+m^{2}\right)^{1 / 2}, \infty\right)\right\}=\varnothing .
$$

Next we turn to the on-shell scattering matrix associated with $Q_{m}$. In view of Theorem 2.5(i) and (3.5), the Jost solutions of $Q_{m}$ are given by

$$
\begin{aligned}
& \Psi_{1, \pm}\left(Z, k_{ \pm}, x\right)=\left(\begin{array}{c}
f_{1, \pm}\left(k_{ \pm}, x\right) \\
(Z+m)^{-1}\left(A f_{1, \pm}\right)\left(k_{ \pm}, x\right)
\end{array}\right), \\
& \Psi_{2, \pm}\left(Z, k_{ \pm}, x\right)=\left(\begin{array}{c}
-(Z+m)^{-1}\left(A^{*} f_{2, \pm}\right)\left(k_{ \pm}, x\right) \\
f_{2, \pm}\left(k_{ \pm}, x\right)
\end{array}\right),
\end{aligned}
$$

$$
\begin{aligned}
& \left(Q_{m} \Psi_{j, \pm}\right)\left(Z, k_{ \pm}, x\right)=(-1)^{j+1} Z \Psi_{j, \pm}\left(Z, k_{ \pm}, x\right), \\
& k_{ \pm}=\left(z-\phi_{ \pm}\right)^{2}, \quad \operatorname{Im} k_{ \pm} \geq 0, \quad Z^{2}=z+m^{2}, \quad z \in \mathbb{C} \backslash\{0\}, \quad j=1,2 .
\end{aligned}
$$

In analogy to Theorem 3.2 , the unitary on-shell scattering matrix $S(E)$ corresponding to $Q_{m}$ then reads

Theorem 4.2. Assume (H.3.1). Then if

(i) $|E|>\left(\phi_{+}^{2}+m^{2}\right)^{1 / 2}: S(E)$ in $\mathbb{C}^{2}$ is given by

$$
S(E)=\left(\begin{array}{cc}
T(E) & R^{r}(E) \\
R^{l}(E) & T(E)
\end{array}\right),
$$

where

$$
\begin{aligned}
T(E) & =\left[2 i\left(k_{+} k_{-}\right)^{1 / 2} /(|E|+m)\right] / W\left(\Psi_{-}\left(E, k_{-}\right), \Psi_{+}\left(E, k_{+}\right)\right), \\
R^{l}(E) & =-W\left(\Psi_{-}\left(E,-k_{-}\right), \Psi_{+}\left(E, k_{+}\right)\right) / W\left(\Psi_{-}\left(E, k_{-}\right), \Psi_{+}\left(E, k_{+}\right)\right), \\
R^{r}(E) & =-W\left(\Psi_{-}\left(E, k_{-}\right), \Psi_{+}\left(E,-k_{+}\right)\right) / W\left(\Psi_{-}\left(E, k_{-}\right), \Psi_{+}\left(E, k_{+}\right)\right), \\
k_{ \pm} & =\left(\lambda-\phi_{ \pm}^{2}\right)^{1 / 2}, \quad E^{2}=\lambda+m^{2}>\phi_{+}^{2}+m^{2} .
\end{aligned}
$$

(ii) $\left(\phi_{-}^{2}+m^{2}\right)^{1 / 2}<|E|<\left(\phi_{+}^{2}+m^{2}\right)^{1 / 2}$ :

$$
S(E)=-\overline{W\left(\Psi_{-}\left(E, k_{-}\right), \Psi_{+}\left(E, k_{+}\right)\right)} / W\left(\Psi_{-}\left(E, k_{-}\right), \Psi_{+}\left(E, k_{+}\right)\right) .
$$


Here

$$
\Psi_{ \pm}\left(E, k_{ \pm}, x\right):= \begin{cases}\Psi_{1, \pm}\left(E, k_{ \pm}, x\right), & E>\left(\phi_{-}^{2}+m^{2}\right)^{1 / 2}, \\ \Psi_{2, \pm}\left(-E, k_{ \pm}, x\right), & E<-\left(\phi_{-}^{2}+m^{2}\right)^{1 / 2}\end{cases}
$$

and $W(\Phi, \Psi)$ denotes the determinant of $\Phi, \Psi \in \mathbb{C}^{2}$.

Taking into account the identity

$$
\begin{array}{r}
W\left(\Psi_{-}\left(E, \sigma k_{-}\right), \Psi_{+}\left(E, \sigma^{\prime} k_{+}\right)\right)=W\left(f_{1,-}\left(\sigma k_{-}\right), f_{1,+}\left(\sigma^{\prime} k_{+}\right)\right) /(|E|+m), \\
\sigma, \sigma^{\prime}= \pm 1, E>\left(\phi_{-}^{2}+m^{2}\right)^{1 / 2},
\end{array}
$$

and similarly for $E<-\left(\phi_{-}^{2}+m^{2}\right)^{1 / 2}$, we finally obtain

Theorem 4.3. Assume (H.3.1). Then

$$
S(E)= \begin{cases}S_{1}\left(E^{2}-m^{2}\right), & E>\left(\phi_{-}^{2}+m^{2}\right)^{1 / 2}, \\ S_{2}\left(E^{2}-m^{2}\right), & E<-\left(\phi_{-}^{2}+m^{2}\right)^{1 / 2} .\end{cases}
$$

Next we briefly discuss systems satisfying (H.3.2). Theorems 2.5(i) and 3.6 imply

Theorem 4.4. Assume (H.3.2). Then the spectrum of $Q_{m}$ is purely absolutely continuous, symmetric with respect to the origin, and of multiplicity two. Moreover,

$$
\begin{gathered}
\sigma\left(Q_{m}\right)=\bigcup_{n \in \mathbb{Z} \backslash\{0\}} \Sigma_{n}, \\
\Sigma_{n}=\left[\left|E_{2(n-1)}+m^{2}\right|^{1 / 2},\left|E_{2 n-1}+m^{2}\right|^{1 / 2}\right], \quad \Sigma_{-n}=-\Sigma_{n}, n \in \mathbb{N},
\end{gathered}
$$

with $E_{n}, n \in \mathbb{N}_{0}$, given by (3.39), and

$$
\sigma_{p}\left(Q_{m}\right)=\sigma_{\text {sc }}\left(Q_{m}\right)=\varnothing, \quad m \in \mathbb{R}
$$

We also state

Lemma 4.5. Assume (H.3.2). Let $F_{Q_{m}}(Z), Z \in \mathbb{C}$, be the discriminant of $Q_{m}$. Then (cf. (3.30))

$$
F_{Q_{m}}(Z)=F\left(Z^{2}-m^{2}\right), \quad Z \in \mathbb{C} .
$$

Proof. Similar to (4.4) and (4.9) we get a fundamental matrix $\Phi_{m}$ for $Q_{m}$ :

$$
\begin{aligned}
& \Phi_{m}(E, x)=\left(\begin{array}{cc}
\theta_{1}(\lambda, x)-\phi(0) \varphi_{1}(\lambda, x) & (E+m) \varphi_{1}(\lambda, x) \\
(E+m)^{-1}\left[A\left(\theta_{1}-\phi(0) \varphi_{1}\right)\right](\lambda, x) & \left(A \varphi_{1}\right)(\lambda, x)
\end{array}\right), \\
& \Phi_{m}(E, x)=\left(\begin{array}{ccc}
-\left(A^{*} \varphi_{2}\right)(\lambda, x) & -(E+m)^{-1}\left[A^{*}\left(\theta_{2}+\phi(0) \varphi_{2}\right)\right](\lambda, x) \\
(E+m) \varphi_{2}(\lambda, x) & \theta_{2}(\lambda, x)+\phi(0) \varphi_{2}(\lambda, x)
\end{array}\right), \\
& E<-m, \lambda=E^{2}-m^{2},
\end{aligned}
$$


with $\theta_{j}, \varphi_{j}, j=1,2$, defined in (3.27) and

$$
\Phi_{m}(E, 0)=1, \quad E \in \mathbb{R} \backslash[-m, m] .
$$

Using (3.30), we obtain

$$
F_{Q_{m}}(E):=2^{-1} \operatorname{Tr}\left[\Phi_{m}(E, a)\right]=F(\lambda), \quad E \in \mathbb{R} \backslash[-m, m] .
$$

Analytic continuation w.r.t. $E$ of both sides in (4.17) then yields (4.14).

For further results in the periodic case see Theorem 9.3.

\section{Relative SCATTERING}

In this section we consider relative scattering, i.e., scattering for the pair $\left(H_{j}+W, H_{j}\right)$ with $H_{j}, j=1,2$, satisfying (H.3.1) or (H.3.2) and $W$ satisfying hypothesis

$$
W \in L^{\infty}(\mathbb{R}) \cap L^{1}\left(\mathbb{R} ;\left(1+x^{2}\right) d x\right) \text { real-valued. }
$$

By (H.5.1), $W$ is a relatively compact perturbation of $H_{j}$ and hence

$$
\widetilde{H}_{j}=H_{j}+W, \quad \mathfrak{D}\left(\widetilde{H}_{j}\right)=\mathfrak{D}\left(H_{j}\right), \quad j=1,2,
$$

is well defined. Let $\tilde{f}_{j, \pm}\left(p_{ \pm}, x\right)$ denote the perturbed Jost solutions of $\widetilde{H}_{j}$ defined by

$$
\begin{gathered}
\tilde{f}_{j, \pm}\left(p_{ \pm}, x\right)=f_{j, \pm}\left(p_{ \pm}, x\right) \\
+\int_{x}^{ \pm \infty} d x^{\prime} g_{j}\left(p_{ \pm}, x, x^{\prime}\right) W\left(x^{\prime}\right) \tilde{f}_{j, \pm}\left(p_{ \pm}, x^{\prime}\right), \quad j=1,2, \\
g_{j}\left(p_{ \pm}, x, x^{\prime}\right):=\left[W\left(f_{j,-}\left(p_{-}\right), f_{j,+}\left(p_{+}\right)\right)\right]^{-1} \\
\cdot\left[f_{j,+}\left(p_{+}, x^{\prime}\right) f_{j,-}\left(p_{-}, x\right)\right. \\
\left.\quad-f_{j,+}\left(p_{+}, x\right) f_{j,-}\left(p_{-}, x^{\prime}\right)\right], \quad j=1,2, \\
p_{ \pm}:= \begin{cases}k_{ \pm} & \text {in case (H.3.1), } \\
k & \text { in case (H.3.2). }\end{cases}
\end{gathered}
$$

We also define

$$
\begin{aligned}
\mp W\left(p_{\mp}\right): & :=W\left(\tilde{f}_{j, \mp}\left(-p_{\mp}\right), \tilde{f}_{j, \mp}\left(p_{\mp}\right)\right) \\
& =W\left(f_{j, \mp}\left(-p_{\mp}\right), f_{j, \mp}\left(p_{\mp}\right)\right) \\
& = \begin{cases}\mp 2 i k_{\mp} & \text { in case }(\text { H.3.1) } \\
\pm i \sin (k a) / F_{z}(z(k)) & \text { in case }(\text { H.3.2.2). }\end{cases}
\end{aligned}
$$

Concerning spectral properties of $\widetilde{H}_{j}$ we recall 
Theorem 5.1. Assume (H.5.1) and either (H.3.1) or (H.3.2). Then

$$
\sigma_{\text {ess }}\left(\widetilde{H}_{j}\right)=\sigma_{\text {ac }}\left(\widetilde{H}_{j}\right)=\sigma_{\text {ess }}\left(H_{j}\right), \quad \sigma_{\mathrm{sc}}\left(\widetilde{H}_{j}\right)=\varnothing, \quad j=1,2,
$$

and the multiplicity of $\sigma_{\mathrm{ess}}\left(\tilde{H}_{j}\right)$ coincides with that of $\sigma_{\mathrm{ess}}\left(H_{j}\right), j=1,2$. In addition, the (necessarily simple) eigenvalues of $\widetilde{H}_{j}$ are determined by

$$
W\left(\tilde{f}_{j,-}\left(p_{j,-, l}\right), \tilde{f}_{j,+}\left(p_{j,+, l}\right)\right)=0, \quad j=1,2, l=1,2, \ldots
$$

Moreover, there are no eigenvalues embedded in the essential spectrum and there are no threshold eigenvalues, i.e.,

$$
\sigma_{p}\left(\tilde{H}_{j}\right) \cap \sigma_{\text {ess }}\left(H_{j}\right)=\varnothing, \quad j=1,2 .
$$

Next we turn to the relative on-shell scattering matrix $S_{j \text {, rel }}(\lambda)$ w.r.t. the pair $\left(\widetilde{H}_{j}, H_{j}\right), j=1,2$.

Theorem 5.2. Assume (H.3.1) and (H.5.1). Let $\widetilde{S}_{j}(\lambda), \lambda>\phi_{-}^{2}$, denote the onshell scattering matrix associated with $\widetilde{H}_{j}$. Then $\widetilde{S}_{j}(\lambda)$ is given by (3.11)-(3.13) with $f_{j, \pm}\left(\sigma k_{ \pm}\right)$replaced by $\tilde{f}_{j, \pm}\left(\sigma k_{ \pm}\right), \sigma= \pm 1, j=1,2$, and

$$
S_{j, \text { rel }}(\lambda)=\widetilde{S}_{j}(\lambda) S_{j}(\lambda)^{-1}, \quad \lambda>\phi_{-}^{2}, j=1,2 .
$$

Proof. For simplicity we assume that $\phi_{+}=\phi_{-}=0$. To fix notations, let $A, B, C$ be selfadjoint operators in a separable, complex Hilbert space $\mathfrak{H}$ and suppose $\left(B-z_{0}\right)^{-1}-\left(A-z_{0}\right)^{-1} \in \mathfrak{B}_{1}(\mathfrak{H}),\left(C-z_{0}\right)^{-1}-\left(A-z_{0}\right)^{-1} \in \mathfrak{B}_{1}(\mathfrak{H})$ for some $z_{0} \in \mathbb{C} \backslash \mathbb{R}$. (Here $\mathfrak{B}_{1}(\cdot)$ denotes the set of trace class operators.) Then the wave operators

$$
\Omega_{ \pm}(B, A):=\mathrm{s}-\lim _{t \rightarrow \pm \infty} e^{i t B} e^{-i t A} P_{\mathrm{ac}}(A)
$$

exist $\left(P_{\mathrm{ac}}(A)\right.$ is the projection onto the absolutely continuous subspace of $\left.A\right)$ and the scattering operator

$$
S(B, A):=\Omega_{+}(B, A)^{*} \Omega_{-}(B, A)
$$

is unitary in $P_{\mathrm{ac}}(A) \mathfrak{H}$. In view of the applications below, assume $L^{2}\left((0, \infty) ; \mathbb{C}^{2}\right)$ to be the spectral representation and $U_{A}^{ \pm}: P_{\mathrm{ac}}(A) \mathfrak{H} \rightarrow L^{2}\left((0, \infty) ; \mathbb{C}^{2}\right.$ ) (constructed from the generalized eigenfunctions of $A$ ) the spectral transformations of $A P_{\mathrm{ac}}(A)$, i.e.,

$$
\left(U_{A}^{ \pm} A P_{\mathrm{ac}}(A) g\right)(k)=k^{2}\left(U_{A}^{ \pm} g\right)(k), \quad k \in(0, \infty), g \in \mathfrak{D}\left(A P_{\mathrm{ac}}(A)\right) .
$$

Since $S(B, A)$ commutes with $A P_{\mathrm{ac}}(A)$, we get for a.e. $k \in(0, \infty)$,

$$
\left(U_{A}^{ \pm} S(B, A)\left(U_{A}^{ \pm}\right)^{*} f\right)(k)=S(B, A, k) f(k), \quad f(k) \in \mathbb{C}^{2},
$$

where $S(B, A, k), k>0$, is unitary in $\mathbb{C}^{2}$. Using the chain rule

$$
\Omega_{ \pm}(C, B) \Omega_{ \pm}(B, A)=\Omega_{ \pm}(C, A)
$$


and

$$
\Omega_{ \pm}(B, A)^{*} \Omega_{ \pm}(B, A)=P_{\text {ac }}(A), \quad \Omega_{ \pm}(B, A)=\Omega_{ \pm}(A, B)^{*},
$$

one obtains

$$
S(C, B)=\Omega_{+}(B, A) S(C, A) S(B, A)^{*} \Omega_{+}(B, A)^{*} .
$$

Finally, taking into account

$$
\Omega_{ \pm}(B, A)=\left(U_{B}^{ \pm}\right)^{*} U_{A}^{ \pm}, \quad\left(U_{B}^{ \pm}\right)^{*} U_{B}^{ \pm}=P_{\mathrm{ac}}(B), \quad U_{B}^{ \pm}\left(U_{B}^{ \pm}\right)^{*}=1,
$$

equation (5.16) implies

$$
U_{B}^{+} S(C, B)\left(U_{B}^{+}\right)^{*}=\left[U_{A}^{+} S(C, A)\left(U_{A}^{+}\right)^{*}\right]\left[U_{A}^{+} S(B, A)\left(U_{A}^{+}\right)^{*}\right]^{*} .
$$

Together with (5.13) this yields (5.9) by identifying $C=\widetilde{H}_{j}, B=H_{j}, A=$ $-d^{2} / d x^{2}, \mathfrak{D}(A)=H^{2}(\mathbb{R})$. The general case $\phi_{+} \neq 0, \phi_{-} \neq 0$ can be treated in a similar manner by introducing two comparison dynamics $A_{ \pm}$as $t \rightarrow \pm \infty$ in (5.10) (see, e.g., [96]).

Similarly one gets (see also $[44,46])$

Theorem 5.3. Assume (H.3.2) and (H.5.1). Then

$$
S_{j, \text { rel }}(\lambda)=\left(\begin{array}{cc}
T_{j, \text { rel }}(\lambda) & R_{j, \text { rel }}^{r}(\lambda) \\
R_{j, \text { rel }}^{l}(\lambda) & T_{j, \text { rel }}(\lambda)
\end{array}\right), \quad \lambda \in \sigma\left(H_{j}\right)^{0}, j=1,2,
$$

where

$$
\begin{aligned}
T_{j, \text { rel }}(\lambda) & =W(k) / W\left(\tilde{f}_{j,-}(k), \tilde{f}_{j,+}(k)\right), \\
R_{j, \text { rel }}^{l}(\lambda)=-W\left(\tilde{f}_{j,-}(-k), \tilde{f}_{j,+}(k)\right) / W\left(\tilde{f}_{j,-}(k), \tilde{f}_{j,+}(k)\right), & \\
R_{j, \text { rel }}^{r}(\lambda)=-W\left(\tilde{f}_{j,-}(k), \tilde{f}_{j,+}(-k)\right) / W\left(\tilde{f}_{j,-}(k), \tilde{f}_{j,+}(k)\right), & \quad \lambda \in \sigma\left(H_{j}\right)^{\circ}, j=1,2 .
\end{aligned}
$$

(Here $M^{\circ}$ denotes the interior of a subset $M \subset \mathbb{R}$.) I.e., formally, $S_{j}(\lambda)=1$ in the case of (H.3.2).

Relative scattering theory in connection with the Korteweg-deVries equation has also been studied in [89].

\section{AdDing EIGENVAlues}

As will become clear in $\S 10$, the notion of an $N$-soliton solution of the $\mathrm{KdV}$-equation relative to a nontrivial background $\mathrm{KdV}$-solution is intimately connected with the process of adding $N$-eigenvalues, say $\left\{E_{1}, \ldots, E_{N}\right\}$ to the (background) Hamiltonian $H_{j}=-d^{2} / d x^{2}+V_{j}$ in (3.2), where $\sigma\left(H_{j}\right) \cap$ $\left\{E_{1}, \ldots, E_{N}\right\}=\varnothing$. Moreover, if $\tilde{H}_{j}$ denotes the new Schrödinger operator obtained by this process, then one needs the remaining spectral properties of $H_{j}$ and $\tilde{H}_{j}$ to coincide in the sense that

$$
\sigma\left(\widetilde{H}_{j}\right) \backslash\left\{E_{1}, \ldots, E_{N}\right\}=\sigma\left(H_{j}\right), \quad \sigma_{\text {ess }}\left(\widetilde{H}_{j}\right)=\sigma_{\text {ess }}\left(H_{j}\right)
$$


and similarly for the absolutely continuous spectrum and the spectral multiplicity respectively.

In this section we shall consider two cases relevant in $\S \S 8$ and 10: First the case where $\phi_{+}^{2}=\phi_{-}^{2}$ in (H.3.1) and then the periodic case governed by (H.3.2). (Throughout this section $j$ will be fixed to be either 1 or 2.)

Combining $\S 3$ with Theorems 3.6 and 3.7 of [33] one gets

Theorem 6.1. Assume (H.3.1) with $0 \leq \phi_{-}^{2}=\phi_{+}^{2}=: \phi_{\infty}^{2}$. Let $\lambda_{j, 1}<\lambda_{j, 2}<$ $\cdots<\lambda_{j, N_{j}}, \lambda_{j, l}=\phi_{\infty}^{2}-\kappa_{j, l}^{2}, j=1,2, l=1, \ldots, N_{j}$, denote the eigenvalues of $H_{j}$ and let $\tilde{\kappa}_{j, 1}<\tilde{\kappa}_{j, 2}<\cdots<\tilde{\kappa}_{j, \tilde{N}_{j}}, \alpha_{j, 1}, \alpha_{j, 2}, \ldots, \alpha_{j, \tilde{N}_{j}}$ be arbitrary positive numbers with $\kappa_{j, l} \neq \tilde{\kappa}_{j, m}, l=1, \ldots, N_{j}, m=1, \ldots, \widetilde{N}_{j}$. Then

$$
\begin{aligned}
\widetilde{V}_{j}(x):= & V_{j}(x)-2 \frac{d^{2}}{d x^{2}} \ln W\left(f_{j,+}\left(i \kappa_{j, 1}\right), \ldots, f_{j,+}\left(i \kappa_{j, N_{j}}\right)\right) \\
& -2 \frac{d^{2}}{d x^{2}} \ln W\left(a_{j, 1}, \ldots, a_{j, \widetilde{N}_{j}}\right)
\end{aligned}
$$

(where $W$ denotes the Wronskian determinant [33]), with

$$
\begin{aligned}
a_{j, m}=\frac{W\left(f_{j,+}\left(i \kappa_{j, 1}\right), \ldots, f_{j,+}\left(i \kappa_{j, N_{j}}\right),(-1)^{m+1} f_{j,+}\left(i \tilde{\kappa}_{j, m}\right)+\alpha_{j, m}(-1)^{N_{j}} f_{j,-}\left(i \tilde{\kappa}_{j, m}\right)\right)}{\prod_{l=1}^{N_{j}}\left(\kappa_{j, l}-\tilde{\kappa}_{j, m}\right) W\left(f_{j,+}\left(i \kappa_{j, 1}\right), \ldots, f_{j,+}\left(i \kappa_{j, N_{j}}\right)\right)} \\
\quad m=1, \ldots, \tilde{N}_{j},
\end{aligned}
$$

supports $\tilde{N}_{j}$ bound states $\tilde{\lambda}_{j, \tilde{N}_{j}}<\cdots<\tilde{\lambda}_{j, 1}, \tilde{\lambda}_{j, m}=\phi_{\infty}^{2}-\tilde{\kappa}_{j, m}^{2}, m=1, \ldots$, $\tilde{N}_{j}$, with norming constants

$$
\begin{aligned}
\tilde{c}_{j,+, m}^{2}= & \frac{2(-1)^{m-1} \tilde{\kappa}_{j, m}}{\alpha_{j, m}} \prod_{n \neq m}\left(\frac{\tilde{\kappa}_{j, n}+\tilde{\kappa}_{j, m}}{\tilde{\kappa}_{j, n}-\tilde{\kappa}_{j, m}}\right) \\
& \cdot \prod_{l=1}^{N_{j}}\left(\frac{\tilde{\kappa}_{j, m}-\kappa_{j, l}}{\tilde{\kappa}_{j, m}+\kappa_{j, l}}\right) T_{j}\left(\tilde{\lambda}_{j, m}\right), \quad m=1, \ldots, \tilde{N}_{j},
\end{aligned}
$$

transmission coefficient

$$
\tilde{T}_{j}(\lambda)=\prod_{l=1}^{N_{j}}\left(\frac{k_{-}(\lambda)-i \kappa_{j, l}}{k_{-}(\lambda)+i \kappa_{j, l}}\right) \prod_{m=1}^{\tilde{N}_{j}}\left(\frac{k_{-}(\lambda)+i \tilde{\kappa}_{j, m}}{k_{-}(\lambda)-i \tilde{\kappa}_{j, m}}\right) T_{j}(\lambda),
$$

and reflection coefficients

$$
\tilde{R}_{j}^{l, r}(\lambda)=(-1)^{\tilde{N}_{j}-N_{j}} \prod_{l=1}^{N_{j}}\left(\frac{k_{-}(\lambda)-i \kappa_{j, l}}{k_{-}(\lambda)+i \kappa_{j, l}}\right) \prod_{m=1}^{\tilde{N}_{j}}\left(\frac{k_{-}(\lambda)+i \tilde{\kappa}_{j, m}}{k_{-}(\lambda)-i \tilde{\kappa}_{j, m}}\right) R_{j}^{l, r}(\lambda) .
$$

Remark 6.2. If $\kappa_{j, L}=\tilde{\kappa}_{j, M}$ for some $L, M, 1 \leq L \leq N_{j}, 1 \leq M \leq \tilde{N}_{j}$, (6.3) must be replaced by

(6.7) $a_{j m}=$

$$
\frac{W\left(f_{j,+}\left(i \kappa_{j, L+1 ;}-L\right), \ldots, f_{j,+}\left(i \kappa_{j, N_{j} ;}-L\right),(-1)^{L+1} f_{j,+}\left(i \kappa_{j, L ;}-L\right)+\alpha_{j, M}(-1)^{N_{j}-L} f_{j,-}\left(i \kappa_{j, L ;}-L\right)\right)}{\prod_{l=L+1}^{N_{j}}\left(\kappa_{j, l}-\kappa_{j, L}\right) W\left(f_{j,+}\left(i \kappa_{j, L+1 ;}-L\right), \ldots, f_{j,+}\left(i \kappa_{j, N_{j} ;}-L\right)\right)} .
$$


Here $f_{j, \pm}\left(k_{-;}-L\right)$ are the Jost solutions after having removed the lowest lying $L$ bound states from $V_{j}$, i.e.,

$$
\begin{array}{r}
f_{j, \pm}\left(k_{-;}-L\right)=( \pm 1)^{L} \frac{W\left(f_{j,+}\left(i \kappa_{j, 1}\right), \ldots, f_{j,+}\left(i \kappa_{j, L}\right) ; f_{j \pm}\left(k_{-}\right)\right)}{\prod_{l=1}^{L}\left(i k_{-}+\kappa_{j, l}\right) W\left(f_{j,+}\left(i \kappa_{j, 1}\right), \ldots, f_{j,+}\left(i \kappa_{j, L}\right)\right)}, \\
k_{-} \neq \kappa_{j, L},
\end{array}
$$

and

$$
\begin{aligned}
f_{j, \pm}\left(i \kappa_{j, L ;}-L\right)= & \mp \frac{c_{j, \mp, L}^{2}(-(L-1))}{T_{j}\left(\lambda_{j, L ;}-L\right)} f_{j, \mp}\left(i \kappa_{j, L ;}-(L-1)\right)^{-1} \\
& \cdot \int_{ \pm \infty}^{x} f_{j, \mp}^{2}\left(i \kappa_{j, L ;}-(L-1) d y,\right.
\end{aligned}
$$

with

$$
T_{j}\left(\lambda_{j, L ;}-L\right)=\lim _{k_{-} \rightarrow i \kappa_{j, L}} \prod_{l=1}^{L}\left(\frac{k_{-}-i \kappa_{j, l}}{k_{-}+i \kappa_{j, l}}\right) T_{j}(\lambda)
$$

and

$$
c_{j,+, L}^{2}(-(L-1))=(-1)^{(L-1)} \prod_{l=1}^{L-1}\left(\frac{\kappa_{j, l}-\kappa_{j, L}}{\kappa_{j, l}+\kappa_{j, L}}\right) c_{j,+, L}^{2},
$$

$$
c_{j,-, L}^{2}(-(L-1))=\frac{4 \kappa_{j, L}^{2} T_{j}^{2}\left(\lambda_{j, L ;}-L\right)}{c_{j,+, L}^{2}(-(L-1))} .
$$

Remark 6.3. If $V_{j}$ has no bound states, i.e., $N_{j}=0,(6.2)$ reduces to the formula given by [33]

$$
\widetilde{V}_{j}(x)=V_{j}(x)-2 \frac{d^{2}}{d x^{2}} \ln W\left(a_{j, 1}, \ldots, a_{j, \widetilde{N}_{j}}\right)
$$

with

$$
\text { (6.14) } a_{j, m}=(-1)^{m+1} f_{j,+}\left(i \tilde{\kappa}_{j, m}\right)+\alpha_{j, m} f_{j,-}\left(i \tilde{\kappa}_{j, m}\right), \quad m=1, \ldots, \tilde{N}_{j} \text {. }
$$

For $V_{j} \equiv 0(6.13)$ is equivalent to the $\tilde{N}_{j}$-soliton formula obtained from the Marchenko equation, i.e.,

$$
\tilde{V}_{j}(x)=-2 \frac{d^{2}}{d x^{2}} \ln \left\{\operatorname{det}\left[1+C_{j, \pm}(x)\right]\right\}
$$

(see $[48,57,71,80,106,115])$, where

$$
C_{j, \pm}(x)=\left[c_{j, \pm, l, m}(x)\right]_{l, m=1}^{\widetilde{N}_{j}},
$$

$$
c_{j, \pm, l, m}(x)=\left(\tilde{\kappa}_{j, l}+\tilde{\kappa}_{j, m}\right)^{-1} \tilde{c}_{j, \pm, l} \tilde{c}_{j, \pm, m} \boldsymbol{e}^{\mp\left(\tilde{\kappa}_{j, l}+\tilde{\kappa}_{j, m}\right) x},
$$

$$
l, m=1, \ldots, \tilde{N}_{j} .
$$


The norming constants $\tilde{c}_{j, \pm, m}$ are then related to $\alpha_{j, m}$ via

$$
\begin{aligned}
\alpha_{j, m} & =\frac{2(-1)^{m-1} \tilde{\kappa}_{j, m}}{\tilde{c}_{j,+, m}^{2}} \prod_{n \neq m}^{\tilde{N}_{j}}\left(\frac{\tilde{\kappa}_{j, n}+\tilde{\kappa}_{j, m}}{\tilde{\kappa}_{j, n}-\tilde{\kappa}_{j, m}}\right) \\
& =\frac{(-1)^{m-1} \tilde{c}_{j,-, m}^{2}}{2 \tilde{\kappa}_{j, m}} \prod_{n \neq m}^{\widetilde{N}_{j}}\left(\frac{\tilde{\kappa}_{j, n}-\tilde{\kappa}_{j, m}}{\tilde{\kappa}_{j, n}+\tilde{\kappa}_{j, m}}\right), \quad m=1, \ldots, \tilde{N}_{j} .
\end{aligned}
$$

For the connection between the Marchenko procedure and commutation methods see, e.g., [24].

At the end of this section we briefly consider reflectionless scattering relative to a finite-zone periodic background potential $V_{j}$ fulfilling (H.3.2). Thereby we are looking for potentials $W_{j, \pm}$ giving rise to $\tilde{N}_{j}$ bound states at energies $\tilde{\lambda}_{j, l} \notin \sigma\left(H_{j}\right), l=1, \ldots, \tilde{N}_{j}$,

$$
\tilde{\lambda}_{j, 1}<\tilde{\lambda}_{j, 2}<\cdots<\tilde{\lambda}_{j, \tilde{N}_{j}}, \quad \tilde{N}_{j} \in \mathbb{N},
$$

and zero relative reflection coefficients $R_{j \text {, rel }}^{l}(\lambda)=R_{j, \text { rel }}^{r}(\lambda)=0, \lambda \in \sigma\left(H_{j}\right)$. Assuming the unperturbed potential $V_{j}$ to provide finitely many energy bands $\sigma_{k}, k=1, \ldots, N$, the set of bound states $\left\{\tilde{\lambda}_{j, l} \mid l=1, \ldots, \tilde{N}_{j}\right\}$ decomposes into subsets $\left\{\tilde{\lambda}_{j, l} \mid l=\tilde{N}_{j, k-2}+1, \ldots, \tilde{N}_{j, k-1}\right\},\left(\tilde{N}_{j,-1}=0\right)$ of $\tilde{N}_{j, k-1}$ bound states respectively, sitting in the $(k-1)$ th gap $(k=1, \ldots, N)$. Applying the Marchenko method [80] we have to solve the integral equation

$$
\begin{aligned}
& K_{j, \pm}\left(x, x^{\prime}\right)+\Omega_{j, \pm}\left(x, x^{\prime}\right) \\
& \quad \pm \int_{x}^{ \pm \infty} d y K_{j, \pm}(x, y) \Omega_{j, \pm}\left(y, x^{\prime}\right)=0, \quad x \leq x^{\prime},
\end{aligned}
$$

with kernel

$$
\boldsymbol{\Omega}_{j, \pm}\left(x, x^{\prime}\right)=\sum_{l=1}^{\widetilde{N}_{j}} \tilde{c}_{j, \pm, l}^{2} f_{j, \pm}\left(k_{j, l}, x\right) f_{j, \pm}\left(k_{j, l}, x^{\prime}\right),
$$

where $f_{j, \pm}$ are normalized Floquet solutions belonging to $H_{j}$ (see (3.24)), the quasi-momenta $k_{j, l}$ are related to $\tilde{\lambda}_{j, l}$ via (3.34), and $\tilde{c}_{j, \pm, l}$ are the norming constants corresponding to the $l$ th bound state $\tilde{\lambda}_{j, l}$. For a separable kernel like (6.20) an ansatz of the form

$$
K_{j, \pm}\left(x, x^{\prime}\right)=\sum_{l=1}^{\widetilde{N}_{j}} \tilde{c}_{j, \pm, l} \eta_{j, \pm, l}(x) f_{j, \pm}\left(k_{j, l}, x^{\prime}\right)
$$

reduces (6.19) to a set of algebraic equations

$$
\sum_{m=1}^{\dot{N}_{j}}\left[1+C_{j, \pm}(x)\right]_{l, m} \eta_{j, \pm, m}(x)=-\tilde{c}_{j, \pm, l} f_{j, \pm}\left(k_{j, l}, x\right),
$$


with

$$
\left[C_{j, \pm}(x)\right]_{l, m}= \pm \tilde{c}_{j, \pm, l} \tilde{c}_{j, \pm, m} \int_{x}^{ \pm \infty} d y f_{j, \pm}\left(k_{j, l}, y\right) f_{j, \pm}\left(k_{j, m}, y\right) .
$$

Since for $\left(a_{1}, \ldots, a_{N}\right) \in \mathbb{R}^{N} \backslash\{0\}$

$$
\sum_{l, m=1}^{N} a_{l}\left[C_{j, \pm}(x)\right]_{l m} a_{m}= \pm \int_{x}^{ \pm \infty} d y\left[\sum_{l=1}^{N} a_{l} \tilde{c}_{j, \pm, l} f_{j, \pm}\left(k_{j, l}, y\right)\right]^{2}>0,
$$

$C_{j, \pm}(x)$ is positive definite. Therefore (6.22) can be inverted to give

$$
\begin{aligned}
& K_{j, \pm}\left(x, x^{\prime}\right)=-\sum_{l, m=1}^{\widetilde{N}_{j}}[1\left.+C_{j, \pm}(x)\right]_{l, m}^{-1} \tilde{c}_{j, \pm, l} \tilde{c}_{j, \pm, m} \\
& \cdot f_{j, \pm}\left(k_{j, l}, x\right) f_{j, \pm}\left(k_{j, m}, x^{\prime}\right) .
\end{aligned}
$$

The potentials $W_{j, \pm}$ are now recovered from $K_{j, \pm}$ via

$$
W_{j, \pm}(x)=\mp 2 \frac{d}{d x} K_{j, \pm}(x, x), \quad x \in \mathbb{R} .
$$

Inserting (6.25) into (6.26) we finally obtain

$$
W_{j, \pm}(x)=-2 \frac{d^{2}}{d x^{2}} \ln \left\{\operatorname{det}\left[1+C_{j, \pm}(x)\right]\right\}, \quad x \in \mathbb{R},
$$

for potentials relative reflectionless w.r.t. a finite-zone periodic background. Note that in general $W_{j,+} \neq W_{j,-}$ (cf. $\left.\S 10\right)$.

\section{COMMUtation, MiURA's tRansformation AND SOlUtions OF THE MKDV-EQUATION}

Using commutation methods, we now take a closer look at the Miura transformation which links solutions of the $\mathrm{mKdV}$ - and $\mathrm{KdV}$-equations. In particular, we provide an explicit construction of $\mathrm{mKdV}$-solutions given a corresponding $\mathrm{KdV}$-solution.

We introduce hypothesis

$$
V, \phi \in C^{\infty}\left(\mathbb{R}^{2}\right) \text { real-valued, } \partial_{x}^{n} V, \partial_{x}^{n} \phi \in L^{\infty}\left(\mathbb{R}^{2}\right), n=0,1
$$

Given (H.7.1), we are interested in studying real-valued solutions of the KdVequation

$$
\mathrm{KdV}(V):=V_{t}-6 V V_{x}+V_{x x x}=0
$$

and of the $\mathrm{mKdV}$-equation

$$
\operatorname{mKdV}(\phi):=\phi_{t}-6 \phi^{2} \phi_{x}+\phi_{x x x}=0
$$

for $(t, x) \in \mathbb{R}^{2}$. 
Remark 7.1. In the following we simply assume the existence of smooth solutions of (7.1) and (7.2). The corresponding initial value problems have been studied, e.g., in $[12,25,26,51,66,68,69,74,80,87,101,111]$ (and references therein).

Introducing the Miura transformation [88]

$$
V_{j}(t, x)=\phi(t, x)^{2}+(-1)^{j} \phi_{x}(t, x), \quad(t, x) \in \mathbb{R}^{2}, j=1,2,
$$

a simple calculation shows that

$$
\operatorname{KdV}\left(V_{j}\right)=\left[2 \phi+(-1)^{j} \partial_{x}\right] \mathrm{mKdV}(\phi), \quad j=1,2 .
$$

Remark 7.2.

(i) Note that (up to smoothness properties) $V_{j}(t, x)$ for fixed $t \in \mathbb{R}$ corresponds to the potential $V_{j}$ of $\S 3$ under hypothesis (H.3.1), resp. (H.3.2), i.e., $V_{1}(t, x)$ and $V_{2}(t, x)$ are related to each other by commutation! This fact seems to have been exploited first by Deift [32] and Adler and Moser [6]. In particular, in [6] it is shown that Miura's transformation (7.3) induces a relation analogous to (7.4) between all higher order $\mathrm{KdV}$ - and $\mathrm{mKdV}$-equations (see the end of this section). Further references exploiting commutation are, e.g., $[7,12,13,42,65,75,82,83,102,118]$.

(ii) In contrast to $\mathrm{KdV}$, the transformation $\phi \rightarrow-\phi$ yields again a solution of the mKdV-equation (7.2). This transformation corresponds to interchanging $V_{1}$ and $V_{2}$ (cf. (7.3)) in the $\mathrm{KdV}$-equation (7.1).

(iii) It has been shown in [3] that rapidly decaying solutions $V(t, x)$ as $|x| \rightarrow \infty$ of (7.1) in general cannot be obtained from rapidly decaying solutions $\phi$ of (7.2) via Miura's transformation (7.3). This explains the necessity of studying solutions $\phi$ of (7.2) with nontrivial spatial asymptotics (see also [48]).

(iv) It is obvious from (7.4) that, whenever $\phi \not \equiv 0$ satisfies (H.7.1) and (7.2), both $V_{1}$ and $V_{2}$ satisfy (7.1) and (H.7.1) and at least one of $V_{1}$ or $V_{2}$ is not identically zero. On the other hand, if $V_{1}$ or $V_{2}$ solve (7.1), we cannot trivially reverse that procedure and construct a solution $\phi$ of (7.2) since $2 \phi \mp \partial_{x}$ on $C^{\infty}\left(\mathbb{R}^{2}\right)$ has the nontrivial kernel

$$
f(t) \exp \left[ \pm 2 \int_{x_{0}}^{x} d y \phi(t, y)\right], \quad f \in C^{\infty}(\mathbb{R}), x_{0} \in \mathbb{R} .
$$

One of the striking features of $V(t, x)$, resp. $\phi(t, x)$, satisfying (7.1), resp. (7.2), is that they induce isospectral deformations of the potential in the Schrödinger, resp. Dirac, operator [77]:

Theorem 7.3. (i) Assume $V$ satisfies (H.7.1) and $V_{t}(t, \cdot) \in L^{\infty}(\mathbb{R}), t \in \mathbb{R}$. If in addition $V(t, x)$ solves (7.1), then the Schrödinger operator $H(t)$ in $L^{2}(\mathbb{R})$,

$$
H(t)=-\partial_{x}^{2}+V(t, \cdot), \quad \mathfrak{D}(H(t))=H^{2}(\mathbb{R}), \quad t \in \mathbb{R},
$$

is unitarily equivalent to $H(0)$ for all $t \in \mathbb{R}$, i.e., there exists a family of unitary operators $U(t), t \in \mathbb{R}, U(0)=1$ in $L^{2}(\mathbb{R})$ such that

$$
U(t)^{-1} H(t) U(t)=H(0), \quad t \in \mathbb{R} .
$$


(ii) Assume $\phi$ satisfies (H.7.1), $\phi_{x x} \in L^{\infty}\left(\mathbb{R}^{2}\right)$, and $\phi_{t}(t, \cdot), \phi_{t x}(t, \cdot) \in$ $L^{\infty}(\mathbb{R}), t \in \mathbb{R}$. If in addition $\phi(t, x)$ solves (7.2), then the Dirac operator $Q_{m}(t)$ in $L^{2}(\mathbb{R}) \otimes \mathbb{C}^{2}$,

$$
\begin{gathered}
Q_{m}(t)=\left(\begin{array}{cc}
m & A(t)^{*} \\
A(t) & -m
\end{array}\right), \\
\mathfrak{D}\left(Q_{m}(t)\right)=H^{1}(\mathbb{R}) \otimes \mathbb{C}^{2}, \quad t \in \mathbb{R}, \quad m \in \mathbb{R}, \\
A(t)=\partial_{x}+\phi(t, \cdot), \quad \mathfrak{D}(A(t))=H^{1}(\mathbb{R}), \quad t \in \mathbb{R},
\end{gathered}
$$

is unitarily equivalent to $Q_{m}(0)$ for all $t \in \mathbb{R}$, i.e., there exists a family of unitary operators $W_{m}(t), t \in \mathbb{R}, W_{m}(0)=1$ in $L^{2}(\mathbb{R}) \otimes \mathbb{C}^{2}$ such that

$$
W_{m}(t)^{-1} Q_{m}(t) W_{m}(t)=Q_{m}(0), \quad(m, t) \in \mathbb{R}^{2} .
$$

Proof. The Lax pair

$$
\begin{aligned}
& L(t)=H(t), \\
& B(t)=-4 \partial_{x}^{3}+6 V(t, \cdot) \partial_{x}+3 V_{x}(t, \cdot), \quad \mathfrak{D}(B(t))=H^{3}(\mathbb{R}), \quad t \in \mathbb{R},
\end{aligned}
$$

together with

$$
\partial_{t} L(t)=[B(t), L(t)], \quad \partial_{t} U(t)=B(t) U(t), \quad U(0)=1
$$

is well known to prove assertion (7.7) (cf., e.g., [39, 77, 78, 80, 93], and the references therein). In order to prove (7.10), one could similarly construct a Lax pair (see, e.g., [107]), however, because of (7.4) and Theorem 2.5(i), (7.10) follows from (7.7).

Remark 7.2(iv) (based on (7.4)) shows how to obtain solutions of the KdVequation (7.1) given solutions of the mKdV-equation (7.2). In the following we concentrate on the reversed procedure, i.e., how to construct solutions $V_{2}$, resp. $\phi$, of (7.1), resp. (7.2), given, e.g., a solution $V_{1}$ of (7.1), such that (H.7.1) and (7.3) are satisfied. After reviewing the results of [50] (see, in particular, Theorem 7.9) we shall apply them in the cases of soliton-like and periodic solutions of the $(\mathrm{m}) \mathrm{KdV}$-equation.

Assume $V_{1}$ satisfies (H.7.1) and the $\mathrm{KdV}$-equation (7.1) and introduce

$$
H_{1}(t)=-\partial_{x}^{2}+V_{1}(t, \cdot), \quad \mathfrak{D}\left(H_{1}(t)\right)=H^{2}(\mathbb{R}), \quad t \in \mathbb{R} .
$$

By Theorem 7.3,

$$
\mathfrak{E}_{1}:=\inf \left[\sigma\left(H_{1}(t)\right)\right]
$$

is $t$-independent. As will become clear in the course of this section, equation (7.3), being a Riccati-type equation for $\phi$ given $V_{j}$, will have nonsingular solutions $\phi$ only if the associated Schrödinger operators $H_{j}(t)=-\partial_{x}^{2}+V_{j}(t, \cdot), j=$ 1,2 , are nonnegative. Thus we assume from now on (cf. also the discussion following (7.76)-(7.82)) that

$$
\mathbb{E}_{1} \geq 0
$$


An important role in our construction is played by positive, distributional solutions $\psi_{1}$ of

$$
\left(H_{1}(t) \psi_{1}(t)\right)(x)=0, \quad(t, x) \in \mathbb{R}^{2}
$$

(i.e., $\left.\psi_{1}(t, \cdot), \psi_{1, x}(t, \cdot) \in A C_{\mathrm{loc}}(\mathbb{R}), t \in \mathbb{R}\right)$. These positive solutions may be classified into principal $\tilde{\psi}_{1, \pm}(t, x)>0$ and nonprincipal $\hat{\psi}_{1, \pm}(t, x)>0$ solutions of (7.16) at $x= \pm \infty$, being defined by [56]

$$
\pm \int_{0}^{ \pm \infty} d x \tilde{\psi}_{1, \pm}(t, x)^{-2}=\infty, \quad \pm \int_{0}^{ \pm \infty} d x \hat{\psi}_{1, \pm}(t, x)^{-2}<\infty,
$$

where $t$ is held fixed at the moment. If $\hat{\psi}_{1, \pm}(t, x)>0$ is a nonprincipal solution of (7.16) at $x= \pm \infty$ then

$$
\tilde{\psi}_{1, \pm}(t, x):= \pm \hat{\psi}_{1, \pm}(t, x) \int_{x}^{ \pm \infty} d x^{\prime} \hat{\psi}_{1, \pm}\left(t, x^{\prime}\right)^{-2}
$$

is principal at $x= \pm \infty$.

Remark 7.4. Fix $t \in \mathbb{R}$ and assume $H_{1}(t) \geq 0$. In the terminology of [100] (cf. also [90]), $H_{1}(t)$ is critical iff $W\left(\tilde{\psi}_{1,-}(t), \tilde{\psi}_{1,+}(t)\right)=0$ and hence $H_{1}(t)$ is subcritical iff $W\left(\tilde{\psi}_{1,-}(t), \tilde{\psi}_{1,+}(t)\right) \neq 0$. Or, equivalently, $H_{1}(t)$ is subcritical iff there exist two linearly independent, positive, distributional zero-energy solutions of $H_{1}(t)$ and hence $H_{1}(t)$ is critical iff it has a unique (up to multiples of constants) positive, distributional zero-energy solution.

We also recall

Lemma 7.5. Assume $V_{1}$ satisfies (H.7.1). Let $0<\psi_{1} \in C^{\infty}\left(\mathbb{R}^{2}\right)$ be a distributional solution of $H_{1}(t) \psi_{1}(t)=0, t \in \mathbb{R}$, and define

$$
\phi(t, x)=-\psi_{1, x}(t, x) / \psi_{1}(t, x), \quad(t, x) \in \mathbb{R}^{2} .
$$

Then $\phi$ satisfies (H.7.1) and $\partial_{x}^{2} \phi \in L^{\infty}\left(\mathbb{R}^{2}\right)$, and $V_{2}=\phi^{2}+\phi_{x}$ satisfies (H.7.1). Proof. As shown in Corollary XI.6.5 of [56], $V_{1} \in L^{\infty}\left(\mathbb{R}^{2}\right)$ yields

$$
\phi \in L^{\infty}\left(\mathbb{R}^{2}\right) \text {. }
$$

Since $V_{1}=\phi^{2}-\phi_{x}$ we infer $\phi_{x} \in L^{\infty}\left(\mathbb{R}^{2}\right)$. The rest is trivial.

Next we study the time dependence of solutions of (7.16) in more detail.

Theorem 7.6. Assume $V_{1}$ satisfies (H.7.1) and the KdV-equation (7.1). Let $\psi_{1, \pm} \in C^{\infty}(\mathbb{R})$ be real-valued, distributional solutions of $H_{1}(0) \psi_{1, \pm}=0$. Then $H_{1}(t) \psi_{1, \pm}(t)=0, t \in \mathbb{R}$, has unique real-valued, distributional solutions $\psi_{1, \pm} \in$ $C^{\infty}\left(\mathbb{R}^{2}\right)$ that evolve according to $U_{1}(t)$ in (7.11), (7.12) (with $H(t) \equiv H_{1}(t)$ ), i.e.,

$$
\begin{aligned}
\psi_{1, \pm, t}(t, x)= & -4 \psi_{1, \pm, x x x}(t, x)+6 V_{1}(t, x) \psi_{1, \pm, x}(t, x) \\
& +3 V_{1, x}(t, x) \psi_{1, \pm}(t, x),
\end{aligned}
$$


or, equivalently, using $\psi_{1, \pm, x x}=V_{1} \psi_{1, \pm}$,

$$
\begin{aligned}
\psi_{1, \pm, t}(t, x)= & 2 V_{1}(t, x) \psi_{1, \pm, x}(t, x) \\
& -V_{1, x}(t, x) \psi_{1, \pm}(t, x), \quad(t, x) \in \mathbb{R}^{2},
\end{aligned}
$$

with

$$
\psi_{1, \pm}(0, x)=\psi_{1, \pm}(x), \quad x \in \mathbb{R} .
$$

In particular, the Wronskian

$$
W\left(\psi_{1,-}(t), \psi_{1,+}(t)\right)=W\left(\psi_{1,-}, \psi_{1,+}\right)
$$

is independent of $(t, x) \in \mathbb{R}^{2}$.

Proof. Consider the Volterra-integral equation

$$
\begin{aligned}
\psi_{1}(t, x)=c(t)+d(t) x+\int_{0}^{x} d x^{\prime}\left(x-x^{\prime}\right) V_{1}\left(t, x^{\prime}\right) \psi_{1}\left(t, x^{\prime}\right), \\
c, d \in C^{\infty}(\mathbb{R}),(t, x) \in \mathbb{R}^{2} .
\end{aligned}
$$

Then, iterating (7.23), one obtains $\psi_{1} \in C^{\infty}\left(\mathbb{R}^{2}\right)$ and $H_{1}(t) \psi_{1}(t)=0$ in the distributional sense. Moreover,

$$
\psi_{1, t x x}-V_{1} \psi_{1, t}=V_{1, t} \psi_{1} .
$$

Next, define

$$
\Psi(t, x):=2 V_{1}(t, x) \psi_{1, x}(t, x)-V_{1, x}(t, x) \psi_{1}(t, x), \quad(t, x) \in \mathbb{R}^{2} .
$$

Then (7.1) implies

$$
\Psi_{x x}-V_{1} \Psi=V_{1, t} \psi_{1}
$$

i.e.,

$$
\left[\Psi-\psi_{1, t}\right]_{x x}=V_{1}\left[\Psi-\psi_{1, t}\right]
$$

Since

$$
\begin{aligned}
\psi_{1, t}(t, 0) & =\dot{c}(t), \quad \psi_{1, t x}(t, 0)=\dot{d}(t), \\
\Psi(t, 0) & =2 V_{1}(t, 0) d(t)-V_{1, x}(t, 0) c(t), \\
\Psi_{x}(t, 0) & =V_{1, x}(t, 0) d(t)+\left[2 V_{1}(t, 0)^{2}-V_{1, x x}(t, 0)\right] c(t), \quad t \in \mathbb{R},
\end{aligned}
$$

we get

$$
\begin{aligned}
\psi_{1, t}(t, x) & =\Psi(t, x) \\
& =2 V_{1}(t, x) \psi_{1, x}(t, x)-V_{1, x}(t, x) \psi_{1}(t, x), \quad(t, x) \in \mathbb{R}^{2},
\end{aligned}
$$

iff

$$
\frac{d}{d t}\left(\begin{array}{l}
c(t) \\
d(t)
\end{array}\right)=\left(\begin{array}{cc}
-V_{1, x}(t, 0) & 2 V_{1}(t, 0) \\
2 V_{1}(t, 0)^{2}-V_{1, x x}(t, 0) & V_{1, x}(t, 0)
\end{array}\right)\left(\begin{array}{l}
c(t) \\
d(t)
\end{array}\right), \quad t \in \mathbb{R} .
$$


Finally (7.22) follows by a simple calculation using $H_{1}(t) \psi_{1, \pm}(t)=0$ and (7.21).

Unless otherwise stated, from now on the time-dependence of distributional solutions $\psi_{1}(t, x)$ of $H_{1}(t) \psi_{1}(t)=0$ is always chosen according to (7.21) (occasionally we also use the notation $\psi_{1}(t, x)=\left(U_{1}(t) \psi_{1}(0)\right)(x)$ to stress that fact). Next we recall

Lemma 7.7 [78]. Assume $V_{1}$ satisfies (H.7.1) and the $K d V$-equation (7.1). Let $\psi_{1} \in C^{\infty}\left(\mathbb{R}^{2}\right)$ be a distributional solution of $H_{1}(t) \psi_{1}(t)=0, t \in \mathbb{R}$, evolving in time according to (7.21). If $\psi_{1}(t, x(t))=0, t \in \mathbb{R}$, then $x(t)$ solves

$$
\dot{x}(t)=-2 V_{1}(t, x), \quad t \in \mathbb{R} .
$$

Conversely, if $\psi_{1}\left(t_{0}, x_{0}\right)=0$, solve

$$
\dot{x}(t)=-2 V_{1}(t, x), \quad t \in \mathbb{R}, x\left(t_{0}\right)=x_{0},
$$

to get $\psi_{1}(t, x(t))=0, t \in \mathbb{R}$. In particular, if $\psi_{1}(0, x)>0, x \in \mathbb{R}$, then

$$
\psi_{1}(t, x)>0, \quad(t, x) \in \mathbb{R}^{2} .
$$

Proof. Equation (7.31) is obvious from $d \psi=0$. Conversely, if $x(t), t \in \mathbb{R}$, is the unique solution of (7.32) (which exists by standard considerations if, e.g., $\left.V_{1} \in C^{1}\left(\mathbb{R}^{2}\right), V_{1}, V_{1, x} \in L^{\infty}\left(\mathbb{R}^{2}\right)\right)$ then

$$
d \psi_{1}=\psi_{1, t} d t+\psi_{1, x} d x=-V_{1, x} \psi_{1} d t
$$

yields $\psi_{1}(t, x(t))=0$ since $\psi_{1}\left(t_{0}, x_{0}\right)=0$. Finally, if $\psi_{1}(0, x)>0, x \in \mathbb{R}$, assume that $\psi_{1}\left(t_{0}, x_{0}\right)=0$ for some $\left(t_{0}, x_{0}\right) \in \mathbb{R}^{2}$. Then, propagating $\psi_{1}$ from $t_{0}$ to 0 in time, $\psi_{1}(0, x(0))$ would be zero as shown above.

Given these results we now may strengthen Remark 7.4.

Lemma 7.8. Assume $V_{1}$ satisfies (H.7.1) and the $K d V$-equation (7.1). Then $H_{1}(t)$ is subcritical (resp. critical) for all $t \in \mathbb{R}$ iff $H_{1}(0)$ is subcritical (resp. critical).

Proof. $H_{1}(0)$ is subcritical iff there exist solutions

$$
\begin{gathered}
\psi_{1, \pm}(0, x)>0, \quad x \in \mathbb{R}, \\
H_{1}(0) \psi_{1, \pm}(0)=0, \quad W\left(\psi_{1,-}(0), \psi_{1,+}(0)\right) \neq 0 .
\end{gathered}
$$

By Theorem 7.6 and Lemma 7.7,$$
\text { (7.36) } \psi_{1, \pm}(t, x):=\left(U_{1}(t) \psi_{1, \pm}(0)\right)(x)>0 \text {, }
$$$$
(t, x) \in \mathbb{R}^{2}, H_{1}(t) \psi_{1, \pm}(t)=0, t \in \mathbb{R},
$$

and

(7.37) $W\left(\psi_{1,-}(t), \psi_{1,+}(t)\right)=W\left(\psi_{1,-}(0), \psi_{1,+}(0)\right) \neq 0, \quad t \in \mathbb{R}$, i.e., $H_{1}(t)$ is subcritical for all $t \in \mathbb{R}$. 
Our main result finally reads [50]

Theorem 7.9. Assume $V_{1}$ satisfies (H.7.1) and the $K d V$-equation (7.1). Let $0<$ $\psi_{1, \pm} \in C^{\infty}\left(\mathbb{R}^{2}\right)$ be positive, distributional solutions of $H_{1}(t) \psi_{1}(t)=0, t \in \mathbb{R}$, evolving in time according to (7.21). Define

$$
\begin{aligned}
\psi_{1, \sigma}(t, x):= & 2^{-1}[1-\sigma(t)] \psi_{1,-}(t, x) \\
& +2^{-1}[1+\sigma(t)] \psi_{1,+}(t, x), \quad(t, x) \in \mathbb{R}^{2}, \\
\phi_{\sigma}(t, x): & =-\psi_{1, \sigma, x}(t, x) / \psi_{1, \sigma}(t, x), \quad(t, x) \in \mathbb{R}^{2}, \\
V_{2, \sigma}(t, x): & =\phi_{\sigma}(t, x)^{2}+\phi_{\sigma, x}(t, x), \quad(t, x) \in \mathbb{R}^{2},
\end{aligned}
$$

where $\sigma: \mathbb{R} \rightarrow[-1,1], \sigma \in C^{\infty}(\mathbb{R})$. Then $\phi_{\sigma}$ and $V_{2, \sigma}$ satisfy $($ H.7.1). In addition,

(7.41) $\operatorname{mKdV}\left(\phi_{\sigma}\right)=0, \quad \operatorname{KdV}\left(V_{2, \sigma}\right)=0$ iff $\dot{\sigma}=0$ or $W\left(\psi_{1,-,} \psi_{1,+}\right)=0$.

Proof. A lengthy, though straightforward computation yields

$$
\begin{aligned}
\operatorname{mKdV}(\phi)=\psi_{1}^{-2}\left\{\psi_{1, t} \psi_{1, x}-\psi_{1} \psi_{1, x t}\right. & -6 V_{1} \psi_{1, x}^{2}+3 \psi_{1, x x}^{2} \\
& \left.+4 \psi_{1, x} \psi_{1, x x x}-\psi_{1} \psi_{1, x x x x}\right\},
\end{aligned}
$$

where $\phi:=-\psi_{1, x} / \psi_{1}$ and $\psi_{1} \in C^{\infty}\left(\mathbb{R}^{2}\right)$ is any positive, distributional solution of $H_{1}(t) \psi_{1}(t)=0$ not necessarily evolving in time according to (7.21). Specializing to $\psi_{1}=\psi_{1, \sigma}$ and taking into account (7.21) for $\psi_{1, \pm}$ finally leads to

$$
\begin{aligned}
& \operatorname{mKdV}\left(\phi_{\sigma}\right)=-\psi_{1, \sigma}^{-2} \dot{\sigma} W\left(\psi_{1,-}, \psi_{1,+}\right) / 2, \\
& \operatorname{KdV}\left(V_{2, \sigma}\right)=\left[2 \phi_{\sigma}+\partial_{x}\right] \operatorname{mKdV}\left(\phi_{\sigma}\right)=2 \psi_{1, \sigma}^{-3} \psi_{1, \sigma, x} \dot{\sigma} W\left(\psi_{1,-}, \psi_{1,+}\right) .
\end{aligned}
$$

Remark 7.10. If $H_{1}(0)$ is critical, we necessarily have $W\left(\psi_{1,-}, \psi_{1,+}\right)=0$ by Remark 7.4 and hence $\phi_{\sigma}$ is actually independent of $\sigma$. If $H_{1}(0)$ is subcritical, then Theorem 7.9 guarantees a one-parameter family of solutions $\phi_{\sigma}, \sigma \in$ $[-1,1]$, of the mKdV-equation (7.2) (choose $\psi_{1, \pm}$ such that $W\left(\psi_{1,-}, \psi_{1,+}\right)$ $\neq 0)$. Moreover, the explicit construction (7.39) yields all smooth solutions $\phi$ of $\mathrm{mKdV}(\phi)=0$ related to $V_{1}$ via $V_{1}=\phi^{2}-\phi_{x}$. (This is obvious in the critical case since the positive solution of $H_{1}(t) \psi_{1}(t)=0$ is unique up to multiples of constants. In the subcritical case choose again $\psi_{1, \pm}$ such that $W\left(\psi_{1,-}, \psi_{1,+}\right) \neq 0$ and observe that $\phi_{\sigma}=-\psi_{1, \sigma, x} / \psi_{1, \sigma}, \sigma \in[-1,1]$, is the general solution of the Riccati-equation $\phi_{x}=\phi^{2}-V_{1}$ on $\mathbb{R}$.) Finally we remark that in the case where $H_{1}$ is critical, $\psi_{1}(t)$ need not necessarily satisfy (7.21) since another (time-dependent) multiple of it (which drops out in the definition of $\phi)$ will satisfy (7.21) by Theorem 7.6.

Remark 7.11. The "if" part in Theorem 7.9 is known and follows, e.g., from prolongation methods developed in [117] (see also [47, 98, 119] and the references therein). In general, these techniques do not distinguish between singular 
and nonsingular solutions $\phi$ of the mKdV-equation. It is the "only if" part in Theorem 7.9 that yields a classification into singular and nonsingular solutions $\phi$ (depending on whether $H_{1}(0)$ is nonnegative or not) and a uniqueness or nonuniqueness result for $\phi$ in Miura's transformation (7.3) depending on whether $H_{1}(0)$ is critical or subcritical. A different approach to this problem can be found in $\S 38$ of [12]. There the authors use the fact that the scattering data for an $n$th order ordinary differential operator can also be viewed as scattering data of a certain first order system. When specializing to Schrödinger operators (i.e., $n=2$ ) and solving an associated matrix factorization problem they are able to treat the Cauchy problem for solutions of the KdV- and $\mathrm{mKdV}$-equations related to each other via Miura's transformation. Their approach assumes rapidly decreasing solutions of the KdV-equation at $\pm \infty$.

Remark 7.12. (i) Given $\phi_{\sigma}$ according to (7.39), $H_{1}(t)$ is recovered from $\phi_{\sigma}$ via

$$
\begin{aligned}
H_{1}(t) & =-\partial_{x}^{2}+V_{1}(t, \cdot)=A_{\sigma}(t)^{*} A_{\sigma}(t), \quad \mathfrak{D}\left(H_{1}(t)\right)=H^{2}(\mathbb{R}), \\
A_{\sigma}(t) & =\partial_{x}+\phi_{\sigma}(t, \cdot), \quad \mathfrak{D}\left(A_{\sigma}(t)\right)=H^{1}(\mathbb{R}), \\
V_{1}(t, x) & =\phi_{\sigma}(t, x)^{2}-\phi_{\sigma, x}(t, x), \quad(t, x) \in \mathbb{R}^{2} .
\end{aligned}
$$

Similarly, $H_{2, \sigma}(t)$ is recovered from $\phi_{\sigma}$ via

$$
\begin{aligned}
& H_{2, \sigma}(t)=A_{\sigma}(t) A_{\sigma}(t)^{*}=-\partial_{x}^{2}+V_{2, \sigma}(t, \cdot), \quad \mathfrak{D}\left(H_{2, \sigma}(t)\right)=H^{2}(\mathbb{R}), \\
& V_{2, \sigma}(t)=\phi_{\sigma}(t, x)^{2}+\phi_{\sigma, x}(t, x), \quad(t, x) \in \mathbb{R}^{2} .
\end{aligned}
$$

(ii) If one starts with $H_{2}(t)$ instead of $H_{1}(t)$, then $\phi_{\sigma}$ is defined by

$$
\phi_{\sigma}(t, x):=\psi_{2, \sigma, x}(t, x) / \psi_{2, \sigma}(t, x), \quad(t, x) \in \mathbb{R}^{2},
$$

where

$$
\begin{aligned}
\psi_{2, \sigma}(t, x):= & 2^{-1}[1-\sigma(t)] \psi_{2,-}(t, x) \\
& +2^{-1}[1+\sigma(t)] \psi_{2,+}(t, x), \quad(t, x) \in \mathbb{R}^{2},
\end{aligned}
$$

and $0<\psi_{2, \pm} \in C^{\infty}\left(\mathbb{R}^{2}\right)$ are positive, distributional solutions of $H_{2}(t) \psi_{2}(t)=0$ evolving in time according to (7.21) (with $V_{1}$ replaced by $V_{2}$ ).

In the case where both $V_{1}$ and $V_{2}$ are given and linked to $\phi$ via Miura's transformation (7.3), one reconstructs $\phi$ as follows:

Lemma 7.13. Assume that $V_{j}, j=1,2$, satisfy (H.7.1) and Miura's transformation (7.3) for some $\phi \in C^{\infty}\left(\mathbb{R}^{2}\right)$. Then $\phi$ satisfies (H.7.1) and

$$
\phi(t, x)=\left\{\left[V_{1, x}(t, x)+V_{2, x}(t, x)\right] / 2\left[V_{2}(t, x)-V_{1}(t, x)\right]\right\}, \quad(t, x) \in \mathbb{R}^{2} .
$$

Moreover, if $V_{1}$ and $V_{2}$ satisfy the KdV-equation (7.1), then $\phi$ satisfies the mKdV-equation (7.2).

Proof. Equation (7.48) follows from

$$
2 \phi_{x}=V_{2}-V_{1},
$$$$
2 \phi^{2}=V_{1}+V_{2},
$$$$
4 \phi \phi_{x}=V_{1, x}+V_{2, x} .
$$

The rest is obvious from (7.4). 
Next we illustrate the content of Theorem 7.9 in two important special cases, viz., soliton-like and periodic solutions $V$ (resp. $\phi)$ of the $(\mathrm{m}) \mathrm{KdV}$-equation For the soliton-like case we introduce hypothesis

(i) $V \in C^{\infty}\left(\mathbb{R}^{2}\right)$ real-valued, $\partial_{x}^{n} V \in L^{\infty}\left(\mathbb{R}^{2}\right), n=0,1, \lim _{x \rightarrow \pm \infty} V(t, x)=$ $V_{ \pm} \in \mathbb{R}, \quad 0<V_{-} \leq V_{+}, \pm \int_{0}^{ \pm \infty} d x\left(1+x^{2}\right)\left|V(t, x)-V_{ \pm}\right|<\infty, t \in \mathbb{R}$.

(ii) $\phi \in C^{\infty}\left(\mathbb{R}^{2}\right)$ real-valued, $\partial_{x}^{n} \phi \in L^{\infty}\left(\mathbb{R}^{2}\right), n=0,1, \lim _{x \rightarrow \pm \infty} \phi(t, x)=$ $\phi_{ \pm} \in \mathbb{R}, 0<\phi_{-}^{2} \leq \phi_{+}^{2}, \pm \int_{0}^{ \pm \infty} d x\left(1+x^{2}\right)\left|\phi(t, x)-\phi_{ \pm}\right|<\infty$, $\int_{\mathbb{R}} d x\left(1+x^{2}\right)\left|\phi_{x}(t, x)\right|<\infty, \quad t \in \mathbb{R}$.

(Here $V_{ \pm}, \phi_{ \pm}$are $t$-independent constants.)

For the periodic case we introduce (H.7.3) $V, \phi \in C^{\infty}\left(\mathbb{R}^{2}\right)$ real-valued,

$$
\begin{aligned}
& \text { for some } a>0: V(t, x+a)=V(t, x), \quad(t, x) \in \mathbb{R}^{2}, \\
& \phi(t, x+a)=\phi(t, x), \quad(t, x) \in \mathbb{R}^{2} .
\end{aligned}
$$

We first study systems governed by (H.7.2). Assume (cf. (7.15))

$$
\mathbb{E}_{1}=0 \quad \text { (i.e., } H_{1}(t), t \in \mathbb{R} \text {, is critical). }
$$

In this case (since $V_{ \pm}>0$ )

$$
H_{1}(t) \psi_{1,0}(t)=0, \quad 0<\psi_{1,0}(t, \cdot) \in H^{2}(\mathbb{R}), t \in \mathbb{R},
$$

has a unique solution $\psi_{1,0} \in C^{\infty}\left(\mathbb{R}^{2}\right)$ up to multiples of constants (this follows, e.g., from nonoscillation theorems [37]) which, without loss of generality (cf. the end of Remark 7.10), may be assumed to evolve according to (7.21). Then one has

$$
\psi_{1,0}(t, x) \underset{x \rightarrow \pm \infty}{=} e^{\mp V_{ \pm}^{1 / 2}\left(x+2 V_{ \pm} t\right)}+o\left(e^{\mp V_{ \pm}^{1 / 2} x}\right), \quad t \in \mathbb{R},
$$

and thus, according to (7.39), defines

$$
\phi_{0}(t, x):=-\psi_{1,0, x}(t, x) / \psi_{1,0}(t, x), \quad(t, x) \in \mathbb{R}^{2} .
$$

Next suppose that

$$
\mathfrak{E}_{1}>0 \text { (i.e., } H_{1}(t), t \in \mathbb{R} \text {, is subcritical). }
$$

In this case we consider

$$
H_{1}(t) \psi_{1, \pm}(t)=0, \quad 0<\psi_{1, \pm}(t, \cdot) \in L_{\mathrm{loc}}^{\infty}(\mathbb{R}), \quad t \in \mathbb{R},
$$

where again $\psi_{1, \pm}$ evolve in time according to (7.21) and hence

$$
\begin{aligned}
& \psi_{1,+}(t, x) \underset{x \rightarrow \pm \infty}{=} e^{-V_{ \pm}^{1 / 2}\left(x+2 V_{ \pm} t\right)}+o\left(e^{-V_{ \pm}^{1 / 2} x}\right), \\
& \psi_{1,-}(t, x)_{x \rightarrow \pm \infty}^{=} e^{V_{ \pm}^{1 / 2}\left(x+2 V_{ \pm} t\right)}+o\left(e^{V_{ \pm}^{1 / 2} x}\right), \quad t \in \mathbb{R},
\end{aligned}
$$


and define $\psi_{1, \sigma}$ and $\phi_{\sigma}$ as in (7.38) and (7.39), i.e.,

$$
\phi_{\sigma}(t, x):=-\psi_{1, \sigma, x}(t, x) / \psi_{1, \sigma}(t, x), \quad(t, x) \in \mathbb{R}^{2} .
$$

Then we have

Theorem 7.14. Assume that $V_{1}$ satisfies (H.7.2(i)). Then $\phi_{\sigma}$, defined in (7.52), resp. (7.55), satisfies (H.7.2(ii)) and $V_{2, \sigma}=\phi_{\sigma}^{2}+\phi_{\sigma, x}, \sigma \in[-1,1]$, satisfies (H.7.2(i)). Moreover,

$$
\phi_{\sigma, \pm}:=\lim _{x \rightarrow \pm \infty} \phi_{\sigma}(t, x)= \begin{cases} \pm V_{ \pm}^{1 / 2}, & \sigma=0, \mathfrak{E}_{1}=0, \\ V_{ \pm}^{1 / 2}, & \sigma=+1, \mathfrak{E}_{1}>0, \\ -V_{ \pm}^{1 / 2}, & \sigma=-1, \mathfrak{E}_{1}>0, \\ \mp V_{ \pm}^{1 / 2}, & \sigma \in(-1,1), \mathfrak{E}_{1}>0,\end{cases}
$$

and

$$
\begin{array}{lrr}
0 \in \sigma_{d}\left(H_{1}(t)\right), \quad 0 \notin \sigma_{d}\left(H_{2, \sigma}(t)\right), & \phi_{\sigma,-}<0<\phi_{\sigma,+}, \\
0 \notin \sigma_{d}\left(H_{1}(t)\right), \quad 0 \in \sigma_{d}\left(H_{2, \sigma}(t)\right), & \phi_{\sigma,+}<0<\phi_{\sigma,-}, \\
0 \notin\left\{\sigma_{d}\left(H_{1}(t)\right) \cup \sigma_{d}\left(H_{2, \sigma}(t)\right)\right\}, & \operatorname{sgn}\left(\phi_{\sigma,-}\right)=\operatorname{sgn}\left(\phi_{\sigma,+}\right), \\
t \in \mathbb{R}, \sigma \in[-1,1] .
\end{array}
$$

Proof. Let $\sigma=0, \mathfrak{E}_{1}=0$. Then standard Volterra-integral equation techniques yield (cf., e.g., [49])

$$
\left(\begin{array}{c}
\psi_{1,0}(t, x) \\
\psi_{1,0, x}(t, x)
\end{array}\right) \underset{x \rightarrow \pm \infty}{=}\left(\begin{array}{c}
1 \\
\mp V_{ \pm}^{1 / 2}
\end{array}\right) e^{\mp V_{ \pm}^{1 / 2}\left(x+2 V_{ \pm} t\right)}+o\left(e^{\mp V_{ \pm}^{1 / 2} x}\right),
$$

$t \in \mathbb{R}$,

and

$$
\phi_{0}(t, x) \underset{x \rightarrow \pm \infty}{=} \pm V_{ \pm}^{1 / 2}+O\left(|x|^{-2}\right) g_{ \pm}(t, x), \quad t \in \mathbb{R},
$$

where $g_{ \pm}(t, \cdot) \in L^{1}((0, \pm \infty)), t \in \mathbb{R}$. Thus

$$
\begin{aligned}
& \pm \int_{0}^{ \pm \infty} d x\left(1+|x|^{2}\right)\left|\phi_{0, x}(t, x)\right| \\
& \quad= \pm \int_{0}^{ \pm \infty} d x\left(1+|x|^{2}\right)\left|V_{1}(t, x)-\phi_{0}(t, x)^{2}\right| \\
& \quad \leq \pm \int_{0}^{ \pm \infty} d x\left(1+|x|^{2}\right)\left|V_{1}(t, x)-V_{ \pm}\right| \\
& \quad \pm \int_{0}^{ \pm \infty} d x\left(1+|x|^{2}\right)\left|\phi_{0}(t, x)^{2}-V_{ \pm}\right|<\infty, \quad t \in \mathbb{R},
\end{aligned}
$$

by (H.7.2(i)) and (7.59). Consequently, also

$$
\pm \int_{0}^{ \pm \infty} d x\left(1+|x|^{2}\right)\left|\phi_{0}(t, x) \mp V_{ \pm}^{1 / 2}\right|<\infty, \quad t \in \mathbb{R}
$$


Similarly,

$$
\begin{aligned}
& \pm \int_{0}^{ \pm \infty} d x\left(1+|x|^{2}\right)\left|V_{2,0}(t, x)-V_{ \pm}\right| \\
& \quad \leq \pm \int_{0}^{ \pm \infty} d x\left(1+|x|^{2}\right)\left|\phi_{0}(t, x)^{2}-V_{ \pm}\right| \\
& \quad \pm \int_{0}^{ \pm \infty} d x\left(1+|x|^{2}\right)\left|\phi_{0, x}(t, x)\right|<\infty, \quad t \in \mathbb{R} .
\end{aligned}
$$

The case $\mathfrak{E}_{1}>0$ follows similarly. (7.57) is discussed, e.g., in [16, 49].

Remark 7.15. A comparison of (7.56) and (7.57) together with Remark 3.8 shows that, whenever $\operatorname{sgn}\left(\phi_{\sigma,-}\right) \neq \operatorname{sgn}\left(\phi_{\sigma_{,}+}\right)$, then $\mathfrak{E}_{1}=0$ or $\mathfrak{E}_{2}=0$ and hence either $H_{1}$ is critical and $H_{2, \sigma}$ is subcritical or vice versa. In particular, $H_{2, \sigma}$ is critical if $\mathfrak{E}_{1}>0, \sigma \in(-1,1)$. Only in the case where $\operatorname{sgn}\left(\phi_{\sigma,-}\right)=$ $\operatorname{sgn}\left(\phi_{\sigma,+}\right)$ one has $\mathfrak{E}_{1}=\mathfrak{E}_{2}>0$ and thus both $H_{1}$ and $H_{2, \sigma}$ are subcritical iff $\sigma= \pm 1$.

Remark 7.16. In order to avoid too many case distinctions in the definition of $\phi_{\sigma}$ we have restricted ourselves to the case $0<V_{-} \leq V_{+}$. Clearly $V_{-}=0$ (i.e., $\left.\sigma_{p}\left(H_{1}(t)\right)=\varnothing, \quad \sigma\left(H_{1}(t)\right)=\sigma_{\mathrm{ac}}\left(H_{1}(t)\right)=[0, \infty), t \in \mathbb{R}\right)$ could be discussed along exactly the same lines by considering zero-energy (i.e., threshold) wave functions of $H_{1}(t)$ behaving asymptotically like $c+d x$ as $|x| \rightarrow \infty$ (see, e.g., $[14,15,72])$.

Remark 7.17. If $V_{j}, j=1,2$, in Lemma 7.13 actually satisfy (H.7.2(i)) then $\phi$ satisfies (H.7.2(ii)) and we get

$$
\begin{aligned}
& \phi_{+}-\phi_{-}=\frac{1}{2} \int_{\mathbb{R}} d y\left[V_{2}(t, y)-V_{1}(t, y)\right], \quad t \in \mathbb{R}, \\
& \phi(t, x)=\phi_{ \pm}-\frac{1}{2} \int_{x}^{ \pm \infty} d y\left[V_{2}(t, y)-V_{1}(t, y)\right], \quad(t, x) \in \mathbb{R}^{2},
\end{aligned}
$$

in addition to (7.48).

Next we turn to systems satisfying (H.7.3). Again we first treat the case

$$
\mathfrak{E}_{1}=0 \text { (i.e., } H_{1}(t), t \in \mathbb{R} \text {, is critical). }
$$

We consider

$$
H_{1}(t) \psi_{1,0}(t)=0, \quad 0<\psi_{1,0}(t, \cdot) \in L^{\infty}(\mathbb{R}), \quad t \in \mathbb{R},
$$

and suppose that $\psi_{1,0}$ evolves in time according to (7.21). By Floquet theory (take $k=0$ in $(3.35)$ ), $\psi_{1,0}$ is periodic in $x$, i.e.,

$$
\psi_{1,0}(t, x+a)=\psi_{1,0}(t, x), \quad(t, x) \in \mathbb{R}^{2},
$$

and we define according to (7.39)

$$
\phi_{0}(t, x):=-\psi_{1,0, x}(t, x) / \psi_{1,0}(t, x), \quad(t, x) \in \mathbb{R}^{2} .
$$


In the case

$$
\mathfrak{E}_{1}>0 \text { (i.e., } H_{1}(t), t \in \mathbb{R} \text {, is subcritical) }
$$

we consider

(7.67)

$$
H_{1}(t) \psi_{1, \pm}(t)=0 \text { (distributional sense), }
$$

$$
0<\psi_{1, \pm}(t, \cdot) \in L_{\text {loc }}^{\infty}(\mathbb{R}), t \in \mathbb{R},
$$

where

$$
\begin{gathered}
\psi_{1, \pm}(t, x) \underset{x \rightarrow \pm \infty}{=} e^{\mp \kappa x}+o\left(e^{\mp \kappa x}\right), \quad t \in \mathbb{R}, \\
\kappa=-i k(0)>0,
\end{gathered}
$$

(cf. (3.34)) and $\psi_{1, \pm}$ satisfy (7.21). In accordance with (7.39) we then define

$$
\phi_{ \pm 1}(t, x):=-\psi_{1, \pm, x}(t, x) / \psi_{1, \pm}(t, x), \quad(t, x) \in \mathbb{R}^{2} .
$$

We have

Theorem 7.18. Assume that $V_{1}$ satisfies (H.7.3). Then $\phi_{\sigma}$, defined in (7.66), resp. (7.70), and $V_{2, \sigma}=\phi_{\sigma}^{2}+\phi_{\sigma, x}, \sigma=0, \pm 1$, both satisfy (H.7.3). In particular,

$$
\begin{aligned}
& \sigma\left(H_{1}(t)\right)=\sigma\left(H_{2, \sigma}(t)\right)=\sigma\left(H_{1}(0)\right), \\
& \sigma_{p}\left(H_{j,(\sigma)}(t)\right)=\varnothing, \quad j=1,2, t \in \mathbb{R}, \sigma=0, \pm 1 .
\end{aligned}
$$

Proof. Because of (7.64), (7.65), (7.67), and (7.68), we have (cf. (3.35))

$$
\psi_{1, \sigma}(t, x) \geq \varepsilon(t)>0, \quad t \in \mathbb{R}, x \in[0, a], \sigma=0, \pm 1 .
$$

Using again standard Volterra-integral equations for $\psi_{1, \sigma}$ one establishes the corresponding differentiability properties of $\psi_{1, \sigma}, \psi_{1, \sigma, x}$ and hence that of $\phi_{\sigma}$.

Remark 7.19. Clearly $\phi_{\sigma}(t, x)$, defined according to (7.39), is not periodic in $x$ for $\sigma \in(-1,1)$ in the subcritical case. Thus we confined ourselves to $\sigma= \pm 1$ in (7.70).

Remark 7.20. In the case where $\mathfrak{E}_{1}=\mathfrak{E}_{2}>0$ we have

$$
\begin{aligned}
& H_{1}=A(t)^{*} A(t)=\widehat{H}_{1}(t)+\mathfrak{E}_{1}, \quad \widehat{H}_{1}(t)=\widehat{A}(t)^{*} \widehat{A}(t), \\
& \widehat{A}(t)=\partial_{x}+\hat{\phi}_{\sigma}(t, \cdot), \quad \mathfrak{D}(\widehat{A}(t))=H^{1}(\mathbb{R}), \quad \sigma= \pm 1, t \in \mathbb{R},
\end{aligned}
$$

where $\hat{\phi}_{\sigma}$ satisfies (H.7.2(ii)) or (H.7.3) and

$$
\begin{array}{r}
\phi_{\sigma}(t, x)^{2}-\phi_{\sigma, x}(t, x)=\hat{\phi}_{\sigma}(t, x)^{2}-\hat{\phi}_{\sigma, x}(t, x)+\mathfrak{E}_{1}, \\
\sigma= \pm 1,(t, x) \in \mathbb{R}^{2} .
\end{array}
$$

In particular, if $V_{j}$ satisfy $(\mathrm{H} .7 .2(\mathrm{i}))$, then $0 \in \sigma_{p}\left(\widehat{H}_{1}(t)\right)$ and

$$
\hat{\phi}_{\sigma, \pm}=\lim _{x \rightarrow \pm \infty} \hat{\phi}_{\sigma}(t, x)= \pm\left(V_{ \pm}-\mathfrak{E}_{1}\right)^{1 / 2} \text {. }
$$

(Similarly, $\hat{\phi}_{\sigma} \rightarrow-\hat{\phi}_{\sigma}$ if $H_{1}(t) \rightarrow H_{2}(t)$.) 
Next we recall a few useful formulas. Define

$$
\begin{aligned}
u_{j}(t, x):=\phi(t, x)^{2}+(-1)^{j} \phi_{x}(t, x)- & \Phi_{0}, \\
& (t, x) \in \mathbb{R}^{2}, \Phi_{0} \in \mathbb{R}, j=1,2 ;
\end{aligned}
$$

then

$$
\operatorname{KdV}\left(u_{j}\right)=\left[2 \phi+(-1)^{j} \partial_{x}\right]\left[\phi_{t}-6\left(\phi^{2}-\Phi_{0}\right) \phi_{x}+\phi_{x x x}\right], \quad j=1,2 .
$$

Next let

$$
y=x-6 \Phi_{0} t, \quad u(t, x):=w(t, y)-\Phi_{0}, \quad(t, x) \in \mathbb{R}^{2}
$$

then

$$
u_{t}-6 u u_{x}+u_{x x x}=w_{t}-6 w w_{y}+w_{y y y} .
$$

(This simple fact reproduces the results in [10].) Similarly, let

$$
\phi(t, x):=\chi(t, y), \quad(t, x) \in \mathbb{R}^{2} ;
$$

then

$$
\phi_{t}-6\left(\phi^{2}-\Phi_{0}\right) \phi_{x}+\phi_{x x x}=\chi_{t}-6 \chi^{2} \chi_{y}+\chi_{y y y} .
$$

Equations (7.76)-(7.81) show that using the methods of this section one can construct solutions to the generalized $\mathrm{mKdV}$-equation

$$
\phi_{t}-6\left(\phi^{2}-\Phi_{0}\right) \phi_{x}+\phi_{x x x}=0, \quad \Phi_{0} \in \mathbb{R} \text {. }
$$

This has been studied in [119] (some special solutions of (7.82) have been considered in [47]). Alternatively one could use the generalized Miura transformation (7.76) together with the Galilei transformation $(t, x) \rightarrow\left(t, y=x-6 \Phi_{0} t\right)$ in $V_{j}$ in order to treat general Hamiltonians $H_{j}(t)$ bounded from below by $\Phi_{0} \in \mathbb{R}, H_{j}(t)=-\partial_{x}^{2}+V_{j}(t, \cdot) \geq \Phi_{0}, j=1,2$.

Finally, we briefly indicate the generalization to higher-order $(\mathrm{m}) \mathrm{KdV}$-equations. These equations are recursively defined by

$$
\begin{aligned}
\mathrm{KdV}_{n}(V) & :=V_{t}-2 X_{n, x}=0, \quad n \in \mathbb{N}_{0}, \\
\operatorname{mKdV}_{n}(\phi) & :=\phi_{t}-Y_{n, x}=0, \quad n \in \mathbb{N}_{0},
\end{aligned}
$$

where

$$
\begin{aligned}
& X_{0}=4, \\
& X_{n+1, x}=-\frac{1}{4} X_{n, x x x}+V X_{n, x}+\frac{1}{2} V_{x} X_{n}, \quad n \in \mathbb{N}_{0}, \\
& Y_{0}=4, \\
& Y_{n+1, x}=-\frac{1}{4} Y_{n, x x x}+\phi^{2} Y_{n, x} \\
& \quad+\phi_{x}\left[\int^{x} d x^{\prime}\left(\phi Y_{n, x^{\prime}}\right)+c_{n}\right], \quad n \in \mathbb{N}_{0} .
\end{aligned}
$$


Here, $c_{n}$ are chosen to be the integration constants in $X_{n}, n \in \mathbb{N}_{0}$. Explicitly we have, e.g.,

$$
\begin{aligned}
X_{0}= & c_{0}=4, \\
X_{1}= & 2 V+c_{1}, \\
X_{2}= & -\frac{1}{2} V_{x x}+\frac{3}{2} V^{2}+\left(c_{1} / 2\right) V+c_{2}, \\
X_{3}= & \frac{1}{8} V_{x x x x}-\frac{5}{4} V V_{x x}-\frac{5}{8} V_{x}^{2}+\frac{5}{4} V^{3} \\
& -\left(c_{1} / 8\right) V_{x x}+\left(3 c_{1} / 8\right) V^{2}+\left(c_{2} / 2\right) V+c_{3}, \\
Y_{0}= & d_{0}=4 \\
Y_{1}= & 4 \phi+d_{1}, \\
Y_{2}= & -\phi_{x x}+2 \phi^{3}+c_{1} \phi+d_{2}, \\
Y_{3}= & \frac{1}{4} \phi_{x x x x}-\frac{5}{2} \phi^{2} \phi_{x x}-\frac{5}{2} \phi \phi_{x}^{2}+\frac{3}{2} \phi^{5} \\
& -\left(c_{1} / 4\right) \phi_{x x}+\left(c_{1} / 2\right) \phi^{3}+c_{2} \phi+d_{3}, \quad c_{j}, d_{j} \in \mathbb{R}, j \in \mathbb{N},
\end{aligned}
$$

etc. Thus,

$$
\begin{aligned}
& \mathrm{KdV}_{0}(V)=V_{t}=0 \\
\mathrm{KdV}_{1}(V)= & V_{t}-4 V_{x}=0 \\
\mathrm{KdV}_{2}(V) \equiv & \mathrm{KdV}(V)=V_{t}+V_{x x x}-6 V V_{x}-c_{1} V_{x}=0 \\
\mathrm{KdV}_{3}(V)= & V_{t}-\frac{1}{4} V_{x x x x x}+\frac{5}{2} V V_{x x x}+5 V_{x} V_{x x}-\frac{15}{2} V^{2} V_{x} \\
& +\left(c_{1} / 4\right) V_{x x x}-\left(3 c_{1} / 2\right) V V_{x}-c_{2} V_{x}=0 \\
\mathrm{mKdV}_{0}(\phi)= & \phi_{t}=0 \\
\mathrm{mKdV}_{1}(\phi)= & \phi_{t}-4 \phi_{x}=0 \\
\mathrm{mKdV}_{2}(\phi) \equiv & \mathrm{mKdV}(\phi)=\phi_{t}+\phi_{x x x}-6 \phi^{2} \phi_{x}-c_{1} \phi_{x}=0 \\
\mathrm{mKdV}_{3}(\phi)= & \phi_{t}-\frac{1}{4} \phi_{x x x x x}+\frac{5}{2} \phi^{2} \phi_{x x x}+10 \phi \phi_{x} \phi_{x x}+\frac{5}{2} \phi_{x}^{3} \\
& -\frac{15}{2} \phi^{4} \phi_{x}+\left(c_{1} / 4\right) \phi_{x x x}-\left(3 c_{1} / 2\right) \phi^{2} \phi_{x}-c_{2} \phi_{x}=0
\end{aligned}
$$

etc. (usually one chooses $c_{j}=d_{j}=0, j \in \mathbb{N}$ ). We end up with

Remark 7.21. As proven in [6], (7.4) extends to the whole $(\mathrm{m}) \mathrm{KdV}_{n}$-hierarchy introduced above, i.e.,

$$
\mathrm{KdV}_{n}\left(V_{j}\right)=\left[2 \phi+(-1)^{j} \partial_{x}\right] \mathrm{mKdV}_{n}(\phi), \quad j=1,2, n \in \mathbb{N}_{0},
$$

where $V_{j}, j=1,2$, and $\phi$ are related by Miura's transformation (7.3). Checking the proof of our main Theorem 7.9 shows that it works as well in the present situation, i.e., Theorem 7.9 extends to the entire $(\mathrm{m}) \mathrm{KdV}_{n}$-hierarchy, $n \in \mathbb{N}_{0}$ (in particular, (7.43) holds with $\mathrm{mKdV}$ replaced by $\mathrm{mKdV}_{n}, n \in \mathbb{N}$ ) assuming $\partial_{x}^{m} V_{1} \in L^{\infty}\left(\mathbb{R}^{2}\right), 0 \leq m \leq 2 n-3, n \geq 2$. 


\section{SOlitons OF THE MKDV-EQUATION}

In this section we rederive the $N$-soliton solutions of the $\mathrm{mKdV}$-equation (originally obtained by Grosse $[54,55]$ ) given the corresponding soliton solutions of the $\mathrm{KdV}$-equation. In contrast to $[54,55]$ we do not use inverse scattering techniques for the Dirac operator.

Assume that $V_{1}(t, x)$ is an $N$-soliton solution of the $\mathrm{KdV}$-equation, i.e., that according to Remark 6.3 (and (7.78), (7.79))

$$
\begin{gathered}
V_{1}(t, x)=V_{\infty}-2 \partial_{x}^{2} \ln \left\{\operatorname{det}\left[1+C_{1, \pm}(t, x)\right]\right\}, \quad(t, x) \in \mathbb{R}^{2}, \\
C_{1, \pm}(t, x)=\left[c_{1, \pm, l, m}(t, x)\right]_{l, m=1}^{N}, \quad N \in \mathbb{N}, \\
c_{1, \pm, l, m}(t, x)=\left(\kappa_{l}+\kappa_{m}\right)^{-1} c_{1, \pm, l}(0) c_{1, \pm, m}(0) e^{\mp\left(\kappa_{l}+\kappa_{m}\right) x} e^{ \pm 4\left(\kappa_{l}^{3}+\kappa_{m}^{3}\right) t} \\
\cdot e^{\mp\left(\kappa_{l}+\kappa_{m}\right) 6 V_{\infty} t}, \quad l, m=1, \ldots, N, \quad(t, x) \in \mathbb{R}^{2}, \\
0<\kappa_{N}<\kappa_{N-1}<\cdots<\kappa_{1}, \quad \kappa_{1}^{2} \leq V_{\infty}, \\
c_{1, \pm, l}(t)=c_{1, \pm, l}(0) e^{ \pm 4 \kappa_{l}^{3} t}, \quad t \in \mathbb{R}, l=1, \ldots, N, \\
H_{1}(t)=-\partial_{x}^{2}+V_{1}(t, \cdot), \quad \mathfrak{D}\left(H_{1}(t)\right)=H^{2}(\mathbb{R}), \quad t \in \mathbb{R}, \\
\sigma_{p}\left(H_{1}(t)\right)=\left\{\lambda_{1, l}:=V_{\infty}-\kappa_{l}^{2} \mid l=1, \ldots, N\right\}, \\
H_{1}(0) f_{1, \pm, l}(0)=\left(V_{\infty}-\kappa_{l}^{2}\right) f_{1, \pm, l}(0), \quad f_{1, \pm, l}(0) \in H^{2}(\mathbb{R}), \\
f_{1, \pm, l}(0, x)_{x \rightarrow \pm \infty}^{=} e^{\mp \kappa_{l} x}+o\left(e^{\mp \kappa_{l} x}\right), \\
\left|c_{1, \pm, l}(0)\right|=\left\|f_{1, \pm, l}(0)\right\|_{2}^{-1}, \quad l=1, \ldots, N .
\end{gathered}
$$

The assumption that $H_{1}(0)$ is critical, i.e.,

$$
\mathfrak{E}_{1}=\inf \left[\sigma\left(H_{1}(0)\right)\right]=0,
$$

then yields

$$
V_{\infty}=\kappa_{1}^{2}, \quad \lambda_{1,1}=0 .
$$

Next we turn to the construction of $H_{2}(t)$. First we note that

$$
\begin{aligned}
& H_{1}(t)=A_{0}(t)^{*} A_{0}(t), \quad t \in \mathbb{R}, \\
& A_{0}(t)=\partial_{x}+\phi_{0}(t, \cdot), \quad \mathfrak{D}\left(A_{0}(t)\right)=H^{1}(\mathbb{R}), \quad t \in \mathbb{R},
\end{aligned}
$$

and (cf. Lemma 7.14), since $\mathfrak{E}_{1}=0$,

$$
\phi_{0, \pm}=\lim _{x \rightarrow \pm \infty} \phi_{0}(t, x)= \pm V_{\infty}^{1 / 2}= \pm \kappa_{1} \text {. }
$$

Since $\kappa_{1}>0$ by hypothesis,

$$
0 \in \sigma_{p}\left(A_{0}(t)\right), \quad 0 \notin \sigma_{p}\left(A_{0}(t)^{*}\right), \quad t \in \mathbb{R} .
$$


Thus commutation implies for $H_{2,0}(t)=A_{0}(t) A_{0}(t)^{*}$,

$$
\begin{aligned}
\sigma_{p}\left(H_{2,0}(t)\right) & =\left\{\lambda_{2, l}=\kappa_{1}^{2}-\kappa_{l}^{2} \mid l=2, \ldots, N\right\} \\
& =\sigma_{p}\left(H_{1}(t)\right) \backslash\{0\}, \quad t \in \mathbb{R} .
\end{aligned}
$$

We also recall that

$$
\sigma_{\text {ess }}\left(H_{j,(0)}(t)\right)=\sigma_{\mathrm{ac}}\left(H_{j,(0)}(t)\right)=\left[V_{\infty}, \infty\right), \quad t \in \mathbb{R}, j=1,2 .
$$

Moreover, since all eigenvalues of $H_{j,(0)}(t), j=1,2$, are simple, we obtain from (3.23),

$$
c_{2, \pm, 0, l}(0)=\left[\left(\kappa_{1}-\kappa_{l}\right) /\left(\kappa_{1}+\kappa_{l}\right)\right]^{1 / 2} c_{1, \pm, l}(0), \quad l=2, \ldots, N .
$$

Thus $V_{2,0}(t, x)$ is an $(N-1)$-soliton solution of the $\mathrm{KdV}$-equation,

$$
V_{2,0}(t, x)=\kappa_{1}^{2}-2 \partial_{x}^{2} \ln \left\{\operatorname{det}\left[1+C_{2, \pm, 0}(t, x)\right]\right\}, \quad(t, x) \in \mathbb{R}^{2},
$$

where

$$
\begin{aligned}
& C_{2, \pm, 0}(t, x)=\left[c_{2, \pm, 0, l, m}(t, x)\right]_{l, m=2}^{N}, \quad N \geq 2, \\
& c_{2, \pm, 0, l, m}(t, x)=\left[\frac{\left(\kappa_{1 \mp} \kappa_{l}\right)\left(\kappa_{1 \mp} \kappa_{m}\right)}{\left(\kappa_{1 \pm} \kappa_{l}\right)\left(\kappa_{1 \pm} \kappa_{m}\right)}\right]^{1 / 2} c_{1, \pm, l, m}(t, x), \\
& l, m=2, \ldots, N,(t, x) \in \mathbb{R}^{2} .
\end{aligned}
$$

It remains to compute $\phi_{0}(t, x)$.

Theorem 8.1. Assume $\mathfrak{E}_{1}=0$ and let

$$
\begin{aligned}
& V_{j,(0)}(t, x)=\kappa_{1}^{2}-2 \partial_{x}^{2} \ln \left\{\operatorname{det}\left[1+C_{j, \pm,(0)}(t, x)\right]\right\}, \\
& \qquad(t, x) \in \mathbb{R}^{2}, j=1,2
\end{aligned}
$$

(cf. (8.1) and (8.17)), be an $N$-soliton, resp. $(N-1)$-soliton, solution for the $K d V$-equation (7.1), $N \in \mathbb{N}$. Let $V_{1}, V_{2,0}$, and $\phi_{0}$ be connected by the Miura transformation (7.3). Then

$$
\begin{aligned}
& \phi_{0}(t, x)= \pm \kappa_{1}+\partial_{x} \ln \left\{\operatorname{det}\left[1+C_{1, \pm}(t, x)\right] / \operatorname{det}\left[1+C_{2, \pm,(0)}(t, x)\right]\right\}, \\
& \phi_{0, \pm}= \pm \kappa_{1} \\
&(t, x) \in \mathbb{R}^{2},
\end{aligned}
$$

is an $(2 N-1)$-soliton solution for the $m K d V$-equation (7.2). Moreover, up to an overall sign, the solutions (8.20) represent all reflectionless potentials of the associated Dirac operator $Q(t), t \in \mathbb{R}$, under the assumption that $Q(0)$ has a zero-eigenvalue, i.e., $0 \in \sigma_{d}(Q(0))$.

Proof. Clearly (7.56), (7.63), and (8.10) imply (8.20). The fact that the $\phi_{0}(t, x)$ in (8.20) are reflectionless potentials of $Q_{m}(t), t \in \mathbb{R}$, is a consequence of (4.11) and $\S 6$. Since $\phi_{0, \pm}= \pm \kappa_{1<}>0$,

$$
0 \in \sigma_{p}\left(A_{0}(t)\right), \quad 0 \notin \sigma_{p}\left(A_{0}(t)^{*}\right), \quad t \in \mathbb{R},
$$


implying (cf. (7.57))

$$
0 \in \sigma_{d}(Q(t)), \quad t \in \mathbb{R} .
$$

Conversely, if $0 \in \sigma_{p}(Q(0))$ and, e.g., $\phi_{0,-}<0<\phi_{0,+}$, then (8.22) holds and by (4.11), $V_{j,(0)}(t, x)$ must be reflectionless potentials for $H_{j,(0)}(t), j=1,2$, of the type (8.19).

Remark 8.2. The symmetry $\phi(t, x) \rightarrow-\phi(t, x)$ of the mKdV-equation (7.2) is connected with the interchange $A(t) \rightarrow A(t)^{*}$, or, equivalently, with $H_{1}(t) \rightarrow$ $H_{2}(t)$. This explains the open overall sign in the last part of Theorem 8.1.

It remains to treat the case where $0 \notin \sigma(Q(t)), t \in \mathbb{R}$, i.e., instead of assumption (8.9) we now assume that $H_{1}(0)$ is subcritical, and, hence,

$$
\mathfrak{E}_{1}>0 \text { (i.e., } \mathfrak{E}_{1}=\mathfrak{E}_{2}>0 \text { ). }
$$

Then (8.1)-(8.8) and (8.11), (8.12) still hold whereas (8.10) turns into

$$
V_{\infty}=\lambda_{1,1}+\kappa_{1}^{2}=\mathfrak{E}_{1}+\kappa_{1}^{2} .
$$

Combining Remarks 7.15 and 8.2 , we may restrict ourselves to $\sigma= \pm 1$ in (7.55) and thus (cf. (7.56))

$$
\phi_{\sigma, \pm}=\lim _{x \rightarrow \pm \infty} \phi_{\sigma}(t, x)= \begin{cases}V_{\infty}^{1 / 2}, & \sigma=+1, \\ -V_{\infty}^{1 / 2}, & \sigma=-1,\end{cases}
$$

implies

$$
\sigma\left(H_{1}(t)\right)=\sigma\left(H_{2, \sigma}(t)\right)=\sigma\left(H_{1}(0)\right), \quad t \in \mathbb{R},
$$

and also the validity of (8.15). It remains to compute the norming constants. We get

(8.27) $c_{2, \pm, \sigma, l}(0)=\left(\frac{\sigma V_{\infty}^{1 / 2} \mp \kappa_{l}}{\sigma V_{\infty}^{1 / 2} \pm \kappa_{l}}\right)^{1 / 2} c_{1, \pm, l}(0), \quad l=1, \ldots, N, \sigma= \pm 1$,

and thus $V_{2, \sigma}(t, x)$ is the $N$-soliton potential

$$
\begin{aligned}
V_{2, \sigma}(t, x)=V_{\infty}-2 \partial_{x}^{2} \ln \left\{\operatorname{det}\left[1+C_{2, \pm, \sigma}(t, x)\right]\right\}, & \\
& \sigma= \pm 1,(t, x) \in \mathbb{R}^{2},
\end{aligned}
$$

where

(8.29)

$$
\begin{aligned}
C_{2, \pm, \sigma}(t, x) & =\left[c_{2, \pm \sigma, l, m}(t, x)\right]_{l, m=1}^{N}, \quad N \in \mathbb{N}, \\
c_{2, \pm, \sigma, l, m}(t, x) & =\left[\frac{\left(\sigma V_{\infty}^{1 / 2} \mp \kappa_{l}\right)\left(\sigma V_{\infty}^{1 / 2} \mp \kappa_{m}\right)}{\left(\sigma V_{\infty}^{1 / 2} \pm \kappa_{l}\right)\left(\sigma V_{\infty}^{1 / 2} \pm \kappa_{m}\right)}\right]^{1 / 2} c_{1, \pm, l, m}(t, x), \\
& \quad \sigma= \pm 1, l, m=1, \ldots N,(t, x) \in \mathbb{R}^{2} .
\end{aligned}
$$

Finally we compute $\phi_{\sigma}$ again. 
Theorem 8.3. Assume $\mathfrak{E}_{1}>0$ and let

$$
V_{j,(\sigma)}(t, x)=V_{\infty}-2 \partial_{x}^{2} \ln \left\{\operatorname{det}\left[1+C_{j, \pm,(\sigma)}(t, x)\right]\right\},
$$

$(t, x) \in \mathbb{R}^{2}, j=1,2, \sigma= \pm 1$ (cf. (8.1) and (8.28)), be $N$-soliton solutions of the $K d V$-equation (7.1), $N \in \mathbb{N}$. Let $V_{1}, V_{2, \sigma}$, and $\phi_{\sigma}, \sigma= \pm 1$, be connected by the Miura transformation (7.3). Then

$$
\begin{aligned}
\phi_{\sigma}(t, x) & =\sigma V_{\infty}^{1 / 2}+\partial_{x} \ln \left\{\operatorname{det}\left[1+C_{1, \pm}(t, x)\right] / \operatorname{det}\left[1+C_{2, \pm, \sigma}(t, x)\right]\right\}, \\
& \sigma= \pm 1,(t, x) \in \mathbb{R}^{2}, \\
\phi_{\sigma, \pm}=\sigma V_{\infty}^{1 / 2}, \quad \sigma= \pm 1 &
\end{aligned}
$$

is a $2 N$-soliton solution of the $m K d V$-equation (7.2). Moreover, up to an overall sign, the solutions (8.31) represent all reflectionless potentials of the associated Dirac operator $Q(t), t \in \mathbb{R}$, under the assumption that $Q(0)$ has no zero-eigenvalue, i.e., $0 \notin \sigma_{d}(Q(0))$.

Proof. Similar to that of Theorem 8.1.

Example 8.4. $N=1$.

(i) $\mathfrak{E}_{1}=\inf \left[\sigma\left(H_{1}(0)\right)\right]=0$.

$$
\begin{aligned}
& V_{1}(t, x)=\kappa_{1}^{2}-2 \kappa_{1}^{2} \cosh ^{-2}\left(\kappa_{1} x+2 \kappa_{1}^{3} t\right), \\
& V_{\infty}=\kappa_{1}^{2}, \quad c_{1, \pm, 1}(0)^{2}=2 \kappa_{1}, \\
& V_{2,0}(t, x)=\kappa_{1}^{2}, \\
& \phi_{0}(t, x)=\kappa_{1} \tanh \left(\kappa_{1} x+2 \kappa_{1}^{3} t\right), \quad \kappa_{1}>0,(t, x) \in \mathbb{R}^{2} .
\end{aligned}
$$

(ii) $\mathfrak{E}_{1}=\inf \left[\sigma\left(H_{1}(0)\right)\right]>0$.

$$
\begin{aligned}
V_{1}(t, x)=\left(\mathfrak{E}_{1}+\kappa_{1}^{2}\right)-2 \kappa_{1}^{2} \cosh ^{-2}\left(\kappa_{1} x+\left(2 \kappa_{1}^{3}+6 \kappa_{1} \mathfrak{E}_{1}\right) t\right), \\
V_{\infty}=\mathfrak{E}_{1}+\kappa_{1}^{2}, \quad c_{1, \pm, 1}(0)^{2}=2 \kappa_{1}, \\
V_{2, \sigma}(t, x)=\left(\mathfrak{E}_{1}+\kappa_{1}^{2}\right)-2 \kappa_{1}^{2} \cosh ^{-2}\left(\kappa_{1} x+\left(2 \kappa_{1}^{3}+6 \kappa_{1} \mathfrak{E}_{1}\right) t+\sigma \frac{1}{2} \ln \gamma_{1}\right), \\
\gamma_{1}:=\left[\left(\mathfrak{E}_{1}+\kappa_{1}^{2}\right)^{1 / 2}+\kappa_{1}\right] /\left[\left(\mathfrak{E}_{1}+\kappa_{1}^{2}\right)^{1 / 2}-\kappa_{1}\right], \quad \sigma= \pm 1, \\
\phi_{\sigma}(t, x)=\sigma\left(\mathfrak{E}_{1}+\kappa_{1}^{2}\right)^{1 / 2}-\sigma \kappa_{1}^{2} \cosh ^{-2}\left(\kappa_{1} x+\left(2 \kappa_{1}^{3}+6 \kappa_{1} \mathfrak{E}_{1}\right) t\right) \\
\cdot\left[\left(\mathfrak{E}_{1}+\kappa_{1}^{2}\right)^{1 / 2}+\sigma \kappa_{1} \tanh \left(\kappa_{1} x+\left(2 \kappa_{1}^{3}+6 \kappa_{1} \mathfrak{E}_{1}\right) t\right)\right]^{-1}, \\
\kappa_{1}>0,(t, x) \in \mathbb{R}^{2}, \sigma= \pm 1 .
\end{aligned}
$$

\section{THE TWO-ZONE MODEL}

In this section we investigate the simplest (nontrivial) finite zone model and illustrate the results of $\S 7$. As a by-product we obtain a generalization of a 
well-known result of Hochstadt's [59] in Theorem 9.3. Let

$$
V_{1}(t, x)=2 \mathfrak{P}\left(x+6 \mathfrak{P}(\omega) t+\omega^{\prime}\right)+\mathfrak{P}(\omega), \quad(t, x) \in \mathbb{R}^{2},
$$

where $\mathfrak{P}(z):=\mathfrak{P}\left(z ; \omega ; \omega^{\prime}\right)$ denotes the Weierstrass $\mathfrak{P}$-function [5] with real half-period $\omega>0$ and purely imaginary half-period $\omega^{\prime}\left(\operatorname{Im} \omega^{\prime}>0\right)$. As is well known $[21,35,36,40,61,78,84,92,93], V_{1}$ satisfies the $\mathrm{KdV}$-equation (7.1), i.e.,

$$
\operatorname{KdV}\left(V_{1}\right)=0 .
$$

The corresponding periodic Schrödinger operator

$$
H_{1}(t)=-\partial_{x}^{2}+V_{1}(t, \cdot), \quad \mathfrak{D}\left(H_{1}(t)\right)=H^{2}(\mathbb{R}), \quad t \in \mathbb{R}
$$

(with period $a=2 \omega$ ), has the absolutely continuous band spectrum [76, 93]

$$
\begin{aligned}
& \sigma\left(H_{1}(t)\right)=\left[0, E_{1}\right] \cup\left[E_{2}, \infty\right), \quad t \in \mathbb{R}, \\
& E_{1}=\mathfrak{P}(\omega)-\mathfrak{P}\left(\omega+\omega^{\prime}\right), E_{2}=\mathfrak{P}(\omega)-\mathfrak{P}\left(\omega^{\prime}\right), \\
& \sigma_{p}\left(H_{1}(t)\right)=\sigma_{\mathrm{sc}}\left(H_{1}(t)\right)=\varnothing, \quad t \in \mathbb{R} .
\end{aligned}
$$

The associated normalized Floquet solutions read [76]

$$
\begin{aligned}
& f_{1, \pm}(k, t, x)= \pm\left[\omega^{-1} \zeta(\omega)+\mathfrak{P}(b)\right]^{-1 / 2} \frac{\sigma\left(x+6 \mathfrak{P}(\omega) t+\omega^{\prime} \pm b\right)}{\sigma\left(x+6 \mathfrak{P}(\omega) t+\omega^{\prime}\right) \sigma( \pm b)} \\
& \cdot \boldsymbol{e}^{\mp\left\{[x+6 \mathfrak{P}(\omega) t] \zeta(b)+\zeta\left(\omega^{\prime}\right) b\right\}} \\
& \text { for } \lambda \in\left[0, E_{1}\right],-i(b-\omega) \in\left[0,-i \omega^{\prime}\right], \\
& f_{1, \pm}(k, t, x)=\left[-\omega^{-1} \zeta(\omega)-\mathfrak{P}(b)\right]^{-1 / 2} \frac{\sigma\left(x+6 \mathfrak{P}(\omega) t+\omega^{\prime} \pm b\right)}{\sigma\left(x+6 \mathfrak{P}(\omega) t+\omega^{\prime}\right) \sigma( \pm b)} \\
& \cdot e^{\mp\left\{[x+6 \mathfrak{P}(\omega) t] \zeta(b)+\zeta\left(\omega^{\prime}\right) b\right\}} \\
& \text { for } \lambda \in\left[E_{2}, \infty\right),-i b \in\left[-i \omega^{\prime}, 0\right),
\end{aligned}
$$

where the Floquet parameter $k$ and the energy $\lambda$ in terms of the parameter $b$ are given by

$$
\begin{aligned}
k(b) & =i \zeta(b)-i \omega^{-1} \zeta(\omega) b, \\
\lambda(b) & =\mathfrak{P}(\omega)-\mathfrak{P}(b) .
\end{aligned}
$$

Here $b$ runs counterclockwise through the perimeter of the fundamental quarter rectangle with vertices $0, \omega, \omega+\omega^{\prime}, \omega^{\prime}$, and $\zeta(z):=\zeta\left(z ; \omega, \omega^{\prime}\right)$ and $\sigma(z):=$ $\sigma\left(z ; \omega, \omega^{\prime}\right)$ are the Weierstrass $\zeta$ - and $\sigma$-functions respectively [5].

At zero-energy we have

$$
\begin{aligned}
H_{1}(t) \psi_{1,0}(t)= & 0, \\
0<\psi_{1,0}(t, x)= & \frac{\sigma\left(x+6 \mathfrak{P}(\omega) t+\omega^{\prime}+\omega\right)}{\sigma\left(x+6 \mathfrak{P}(\omega) t+\omega^{\prime}\right) \sigma(\omega)} \\
& \cdot e^{-\left\{[x+6 \mathfrak{P}(\omega) t] \zeta(\omega)+\zeta\left(\omega^{\prime}\right) \omega\right\}}, \quad(t, x) \in \mathbb{R}^{2},
\end{aligned}
$$

and thus (cf. (7.66) and Theorem 7.9) we get 
Theorem 9.1. Let

$$
V_{1}(t, x)=2 \mathfrak{P}\left(x+6 \mathfrak{P}(\omega) t+\omega^{\prime}\right)+\mathfrak{P}(\omega), \quad(t, x) \in \mathbb{R}^{2},
$$

be a two-zone solution of the $K d V$-equation (7.1) with $\mathfrak{E}_{1}=0$. Let $V_{1}, V_{2,0}$, and $\phi_{0}$ be connected by Miura's transformation (7.3). Then

$$
\begin{aligned}
\phi_{0}(t, x) & =-\psi_{1,0, x}(t, x) / \psi_{1,0}(t, x) \\
& =-\frac{1}{2} \frac{\mathfrak{P}^{\prime}\left(x+6 \mathfrak{P}(\omega) t+\omega^{\prime}\right)}{\mathfrak{P}\left(x+6 \mathfrak{P}(\omega) t+\omega^{\prime}\right)-\mathfrak{P}(\omega)} \\
& =\left[\zeta\left(x+6 \mathfrak{P}(\omega) t+\omega^{\prime}\right)+\zeta(\omega)-\zeta\left(x+6 \mathfrak{P}(\omega) t+\omega+\omega^{\prime}\right)\right], \\
& (t, x) \in \mathbb{R}^{2},
\end{aligned}
$$

satisfies the $m K d V$-equation (7.2), i.e.,

$$
\operatorname{mKdV}\left(\phi_{0}\right)=0
$$

and

$$
\begin{aligned}
V_{2,0}(t, x) & =\phi_{0}(t, x)^{2}+\phi_{0, x}(t, x) \\
& =2 \mathfrak{P}\left(x+6 \mathfrak{P}(\omega) t+\omega+\omega^{\prime}\right)+\mathfrak{P}(\omega), \quad(t, x) \in \mathbb{R}^{2},
\end{aligned}
$$

satisfies the $K d V$-equation (7.1), i.e.,

$$
\operatorname{KdV}\left(V_{2,0}\right)=0 .
$$

The fact that $V_{2,0}$ is just a translate of $V_{1}$, i.e.,

$$
V_{2,0}(t, x)=V_{1}(t, x+\omega), \quad(t, x) \in \mathbb{R}^{2},
$$

is no accident (see [116]). In fact one has

Theorem 9.2 [40, 52, 59]. Let $V \in L_{\mathrm{loc}}^{1}(\mathbb{R})$ be real-valued and periodic with period $2 \omega>0$ and define the form sum $H=-d^{2} / d x^{2}+V$ in $L^{2}(\mathbb{R})$. Then

(i) $H$ has spectrum

$$
\sigma(H)=\left[E_{0}, \infty\right), \quad E_{0} \in \mathbb{R}, \quad \text { iff } \quad V=E_{0} \text { a.e. }
$$

(ii) $H$ has spectrum

$$
\sigma(H)=\left[E_{0}, E_{1}\right] \cup\left[E_{2}, \infty\right), \quad E_{0}<E_{1}<E_{2},
$$

iff

$$
V=2 \mathfrak{P}\left(\cdot+\omega^{\prime}+\alpha\right)+C \text { a.e., } C \in \mathbb{R},
$$

where

(9.16) $\quad \alpha \in \mathbb{R}, \quad E_{0}=C-\mathfrak{P}(\omega), \quad E_{1}=C-\mathfrak{P}\left(\omega+\omega^{\prime}\right), \quad E_{2}=C-\mathfrak{P}\left(\omega^{\prime}\right)$. 
By commutation (cf. Theorem 4.4) this immediately yields

Theorem 9.3. Let $\phi \in A C_{\mathrm{loc}}(\mathbb{R})$ be real-valued and periodic with period $2 \omega>0$ and define in $L^{2}(\mathbb{R}), A=d / d x+\phi, \mathfrak{D}(A)=H^{1}(\mathbb{R})$. Let $Q_{m}=\left(\begin{array}{cc}m & A^{*} \\ A & -m\end{array}\right), m \in$ $\mathbb{R}$, be the corresponding Dirac operator in $L^{2}(\mathbb{R})^{2}$. Then

(i) $Q_{m}$ has spectrum

$$
\begin{aligned}
\sigma\left(Q_{m}\right) & =\left(-\infty,-\left|m^{2}+\phi_{0}^{2}\right|^{1 / 2}\right] \cup\left[\left|m^{2}+\phi_{0}^{2}\right|^{1 / 2}, \infty\right), \quad \phi_{0} \in \mathbb{R}, \\
\text { iff } \phi & = \pm \phi_{0} .
\end{aligned}
$$

(ii) $Q_{m}$ has spectrum

$$
\begin{gathered}
\sigma\left(Q_{m}\right)=\left(-\infty,-\left|m^{2}+E_{2}\right|^{1 / 2}\right] \cup\left[-\left|m^{2}+E_{1}\right|^{1 / 2},-\left|m^{2}+E_{0}\right|^{1 / 2}\right] \\
\cup\left[\left|m^{2}+E_{0}\right|^{1 / 2},\left|m^{2}+E_{1}\right|^{1 / 2}\right] \cup\left[\left|m^{2}+E_{2}\right|^{1 / 2}, \infty\right), \\
0 \leq E_{0}<E_{1}<E_{2},
\end{gathered}
$$

iff

where

$$
\phi= \pm \frac{1}{2} \frac{\mathfrak{P}^{\prime}\left(\cdot+\omega^{\prime}+\alpha\right)-\varepsilon \mathfrak{P}^{\prime}\left(b_{0}\right)}{\mathfrak{P}\left(\cdot+\omega^{\prime}+\alpha\right)-\mathfrak{P}\left(b_{0}\right)},
$$

$$
\begin{array}{cc}
\alpha \in \mathbb{R}, \quad \varepsilon= \pm 1, & E_{0}=\mathfrak{P}\left(b_{0}\right)-\mathfrak{P}(\omega), \\
E_{1}=\mathfrak{P}\left(b_{0}\right)-\mathfrak{P}\left(\omega+\omega^{\prime}\right), & E_{2}=\mathfrak{P}\left(b_{0}\right)-\mathfrak{P}\left(\omega^{\prime}\right) .
\end{array}
$$

Interestingly enough, the potential $\phi$ in (9.18) appears in a recently discovered new class of integrable systems [97] (see also [18]) being generalizations of the Calogero-Moser systems. Periodic, finite-zone Dirac operators have also been studied in [60].

Remark 9.4. Quite generally, one can ask which differential equations do the travelling wave solutions of the $\mathrm{KdV}$-equation, respectively $\mathrm{mKdV}$-equation, $V_{j}(x-\nu t), j=1,2$, resp. $\phi(x-\nu t),(t, x, \nu) \in \mathbb{R}^{3}$, connected via Miura's transformation (7.3), solve. The answer is given by

$$
\begin{aligned}
& V_{j}^{\prime 2}=2 V_{j}^{3}+\nu V_{j}^{2}+a V_{j}+b, \quad j=1,2, \\
& \phi^{\prime 2}=\phi^{4}+\nu \phi^{2}+c \phi+d,
\end{aligned}
$$

where

$$
c^{2}=4 b-2 \nu a, \quad d=-a / 2 .
$$

The sign ambiguity of $c$ in (9.22) has to do with the symmetry $\phi \rightarrow-\phi$ of the $\mathrm{mKdV}$-equation and $a, b, \nu$ need to satisfy additional constraints (e.g., $4 b-2 \nu a \geq 0$ to guarantee real-valued solutions). The explicit example (9.8), (9.9), (9.12) leads to

$$
\begin{aligned}
\nu & =-2\left(E_{1}+E_{2}\right)=-6 \mathfrak{P}(\omega), \\
a & =-2\left(E_{2}-E_{1}\right)^{2}=-2\left[\mathfrak{P}\left(\omega+\omega^{\prime}\right)-\mathfrak{P}\left(\omega^{\prime}\right)\right]^{2}, \\
b & =2\left(E_{1}+E_{2}\right)\left(E_{2}-E_{1}\right)^{2}=6 \mathfrak{P}(\omega)\left[\mathfrak{P}\left(\omega+\omega^{\prime}\right)-\mathfrak{P}\left(\omega^{\prime}\right)\right]^{2}, \\
c & =0, \quad d=\left(E_{2}-E_{1}\right)^{2}=\left[\mathfrak{P}\left(\omega+\omega^{\prime}\right)-\mathfrak{P}\left(\omega^{\prime}\right)\right]^{2} .
\end{aligned}
$$




\section{SOLITONS ON THE TWO-ZONE PERIODIC BACKGROUND}

Here we continue the study of the two-zone model of $\S 9$ and construct solitons relative to this background. These $\mathrm{KdV}$-solutions are then transferred to the $\mathrm{mKdV}$-case. Let

$$
\begin{aligned}
& V_{1}(t, x)=2 \mathfrak{P}\left(x-\nu t+\omega^{\prime}\right)-(\nu / 6), \quad(t, x, \nu) \in \mathbb{R}^{3}, \\
& H_{1}(t)=-\partial_{x}^{2}+V_{1}(t, \cdot), \quad \mathfrak{D}\left(H_{1}(t)\right)=H^{2}(\mathbb{R}), \quad t \in \mathbb{R} .
\end{aligned}
$$

Then $[76,93]$

$$
\operatorname{KdV}\left(V_{1}\right)=0
$$

and

$$
\begin{aligned}
& \sigma\left(H_{1}(t)\right)=\left[E_{0}, E_{1}\right] \cup\left[E_{2}, \infty\right), \quad t \in \mathbb{R}, \\
& E_{0}=-\mathfrak{P}(\omega)-(\nu / 6), \quad E_{1}=-\mathfrak{P}\left(\omega+\omega^{\prime}\right)-(\nu / 6), \\
& E_{2}=-\mathfrak{P}\left(\omega^{\prime}\right)-(\nu / 6), \quad \sigma_{p}\left(H_{1}(t)\right)=\sigma_{\mathrm{sc}}\left(H_{1}(t)\right)=\varnothing, \quad t \in \mathbb{R} .
\end{aligned}
$$

We aim to construct reflectionless potentials $W_{1,+}$, resp. $W_{1,-}$ (i.e., solitons), relative to the background $V_{1}$ that vanish as $x \rightarrow+\infty$, resp. as $x \rightarrow-\infty$. Applying the Marchenko method [80] this is accomplished as follows (see $\S 6$ ): Assuming

$$
\nu \leq-6 \mathfrak{P}(\omega)
$$

in order to guarantee

$$
H_{1}(t) \geq 0,
$$

we suppose $W_{1,+}$, resp. $W_{1,-}$, to support $N$ bound states at energies

$$
0 \leq \tilde{\lambda}_{1,1}<\tilde{\lambda}_{1,2}<\cdots<\tilde{\lambda}_{1, N}, \quad N \in \mathbb{N},
$$

where $\tilde{\lambda}_{1, l}:=\lambda\left(b_{l}\right), l=1, \ldots, N$, and

$$
\begin{array}{ll}
0 \leq \tilde{\lambda}_{1, m}<E_{0} & \left(\text { i.e. } \mathfrak{P}(\omega)<\mathfrak{P}\left(b_{m}\right) \leq-\nu / 6\right), \\
& m=1, \ldots, N_{1}, N_{1} \leq N \text { if } \nu<-6 \mathfrak{P}(\omega)
\end{array}
$$

(otherwise (10.8) is empty),

$$
\begin{aligned}
E_{1}<\tilde{\lambda}_{1, m}<E_{2} \quad\left(\text { i.e. } \mathfrak{P}\left(\omega^{\prime}\right)<\mathfrak{P}\left(b_{m}\right)<\mathfrak{P}\left(\omega+\omega^{\prime}\right)\right), & \\
& m=N_{1}+1, \ldots, N .
\end{aligned}
$$

We also introduce $b_{0} \in(0, \omega)$ such that

$$
\lambda\left(b_{0}\right)=-\mathfrak{P}\left(b_{0}\right)-(\nu / 6)=0 .
$$

Clearly

$$
\mathfrak{P}\left(b_{l}\right) \leq \mathfrak{P}\left(b_{0}\right), \quad l=1, \ldots, N .
$$


In order to compute $W_{1, \pm}$ one then solves the Marchenko equation

$$
\begin{aligned}
& K_{1, \pm}\left(t, x, x^{\prime}\right)+\Omega_{1, \pm}\left(t, x, x^{\prime}\right) \\
& \quad \pm \int_{x}^{ \pm \infty} d y K_{1, \pm}(t, x, y) \Omega_{1, \pm}\left(t, y, x^{\prime}\right)=0, \quad t \in \mathbb{R}, x \leq x^{\prime},
\end{aligned}
$$

with the ansatz

$$
\begin{aligned}
\Omega_{1, \pm}\left(t, x, x^{\prime}\right)=\sum_{l=1}^{N} \tilde{c}_{1, \pm, l}(t)^{2} f_{1, \pm}\left(t, k_{l}, x\right) f_{1, \pm}\left(t, k_{l}, x^{\prime}\right), & \\
& \left(t, x, x^{\prime}\right) \in \mathbb{R}^{3},
\end{aligned}
$$

$$
\begin{aligned}
f_{1, \pm}(t, k, x)= & \frac{\sigma\left(x-\nu t+\omega^{\prime} \pm b\right)}{\sigma\left(x-\nu t+\omega^{\prime}\right) \sigma( \pm b)} e^{\mp\left[\zeta(b)(x-\nu t)+\zeta\left(\omega^{\prime}\right) b\right]} \\
& \cdot \begin{cases} \pm\left[\omega^{-1} \zeta(\omega)+\mathfrak{P}(b)\right]^{-1 / 2}, & \lambda \in(-\infty, \lambda(\bar{b})), \\
{\left[-\omega^{-1} \zeta(\omega)-\mathfrak{P}(b)\right]^{-1 / 2},} & \lambda \in(\lambda(\bar{b}), \infty),(t, x) \in \mathbb{R}^{2}\end{cases}
\end{aligned}
$$

(cf. Remark 10.2 for a discussion of $\bar{b}$ ),

$$
\begin{aligned}
& k(b)=i \zeta(b)-i \omega^{-1} \zeta(\omega) b, \\
& k_{l}:=k\left(b_{l}\right), \quad \lambda(b)=-\mathfrak{P}(b)-(\nu / 6),
\end{aligned}
$$

$$
\tilde{c}_{1, \pm, l}(t)^{2}=\tilde{c}_{1, \pm, l}(0)^{2} e^{\mp 4 \mathfrak{P}^{\prime}\left(b_{l}\right) t}, \quad t \in \mathbb{R}, l=1, \ldots, N .
$$

This yields

$$
K_{1, \pm}\left(t, x, x^{\prime}\right)=-\sum_{l=1}^{N} \tilde{c}_{1, \pm, l}(t)^{2} \tilde{f}_{1, \pm}\left(t, k_{l}, x\right) f_{1, \pm}\left(t, k_{l}, x^{\prime}\right),
$$

where

(10.18) $\tilde{f}_{1, \pm}(t, k, x)$

$$
=f_{1, \pm}(t, k, x) \pm \int_{x}^{ \pm \infty} d x^{\prime} K_{1, \pm}\left(t, x, x^{\prime}\right) f_{1, \pm}\left(t, k, x^{\prime}\right),
$$

$$
(t, x) \in \mathbb{R}^{2},
$$

(10.19)

$$
\begin{aligned}
& \widetilde{H}_{1, \pm}(t) \tilde{f}_{1, \pm}(t, k, x)=\lambda \tilde{f}_{1, \pm}(t, k, x), \quad t \in \mathbb{R} \quad \text { (distributional sense), } \\
& \tilde{H}_{1, \pm}(t) \tilde{f}_{1, \pm, l}(t)=\tilde{\lambda}_{1, l} \tilde{f}_{1, \pm, l}(t), \\
& \tilde{f}_{1, \pm, l}(t, \cdot):=\tilde{f}_{1, \pm}\left(t, k_{l}, \cdot\right) \in H^{2}(\mathbb{R}), \\
& \left|\tilde{c}_{1, \pm, l}(0)\right|=\left\|\tilde{f}_{1, \pm, l}(0)\right\|_{2}^{-1}, \quad l=1, \ldots, N .
\end{aligned}
$$

\section{Here}

(10.20) $\quad \widetilde{H}_{1, \pm}(t)=H_{1}(t)+W_{1, \pm}(t, \cdot), \quad \mathfrak{D}\left(\tilde{H}_{1, \pm}(t)\right)=H^{2}(\mathbb{R}), \quad t \in \mathbb{R}$, 
with

$$
\begin{aligned}
W_{1, \pm}(t, x) & =\mp 2 \partial_{x} K_{1, \pm}(t, x, x) \\
& =-2 \partial_{x}^{2} \ln \left\{\operatorname{det}\left[1+\tilde{C}_{1, \pm}(t, x)\right]\right\}, \quad(t, x) \in \mathbb{R}^{2},
\end{aligned}
$$

$$
\begin{aligned}
\tilde{C}_{1, \pm}(t, x)=\left[\tilde{c}_{1, \pm, l, m}(t, x)\right]_{l, m=1}^{N}, \quad N \in \mathbb{N}, & \\
\tilde{c}_{1, \pm, l, m}(t, x)= & \pm \tilde{c}_{1, \pm, l}(t) \tilde{c}_{1, \pm, m}(t) \\
& \cdot \int_{x}^{ \pm \infty} d x^{\prime} f_{1, \pm}\left(t, k_{l}, x^{\prime}\right) f_{1, \pm}\left(t, k_{m}, x^{\prime}\right) \\
= & \tilde{c}_{1, \pm, l}(t) \tilde{c}_{1, \pm, m}(t) f_{1, \pm}\left(t, k_{l}, x\right) f_{1, \pm}\left(t, k_{m}, x\right) \\
& \cdot \frac{\sigma\left(x-\nu t+\omega^{\prime} \pm b_{l} \pm b_{m}\right) \sigma\left(x-\nu t+\omega^{\prime}\right) \sigma\left(b_{l}\right) \sigma\left(b_{m}\right)}{\sigma\left(x-\nu t+\omega^{\prime} \pm b_{l}\right) \sigma\left(x-\nu t+\omega^{\prime} \pm b_{m}\right) \sigma\left(b_{l}+b_{m}\right)}, \\
& l, m=1, \ldots, N,(t, x) \in \mathbb{R}^{2} .
\end{aligned}
$$

Finally, we note that

$$
\operatorname{KdV}\left(\tilde{V}_{1, \pm}\right)=0, \quad \tilde{V}_{1, \pm}=V_{1}+W_{1, \pm} .
$$

In order to shorten the discussion in the following, we only discuss the special case

$$
N=1, \quad 0 \leq \tilde{\lambda}_{1,1}<E_{0}, \quad \nu<-6 \mathfrak{P}(\omega)
$$

in some detail. Clearly (10.17) and (10.18) imply

$$
\begin{aligned}
\tilde{f}_{1, \pm}(t, k, x)=\left[1 \pm \tilde{c}_{1, \pm, 1}(t)^{2} \int_{x}^{ \pm \infty} d x^{\prime} f_{1, \pm}\left(t, k_{1}, x^{\prime}\right)^{2}\right]^{-1} \\
\quad\left\{f_{1, \pm}(t, k, x) \pm \tilde{c}_{1, \pm, 1}(t)^{2}\right. \\
\quad \cdot \int_{x}^{ \pm \infty} d x^{\prime}\left[f_{1, \pm}(t, k, x) f_{1, \pm}\left(t, k_{1}, x^{\prime}\right)\right. \\
\left.\left.\quad-f_{1, \pm}\left(t, k_{1}, x\right) f_{1, \pm}\left(t, k, x^{\prime}\right)\right] f_{1, \pm}\left(t, k_{1}, x^{\prime}\right)\right\}, \quad(t, x) \in \mathbb{R}^{2} .
\end{aligned}
$$

In particular,

$$
\begin{aligned}
\tilde{f}_{1, \pm}\left(t, k_{1}, x\right)= & {\left[1 \pm \tilde{c}_{1, \pm, 1}(t)^{2} \int_{x}^{ \pm \infty} d x^{\prime} f_{1, \pm}\left(t, k_{1}, x^{\prime}\right)^{2}\right]^{-1} } \\
& \cdot f_{1, \pm}\left(t, k_{1}, x\right), \quad(t, x) \in \mathbb{R}^{2}
\end{aligned}
$$

and thus

$$
\tilde{f}_{1, \pm}\left(t, k_{1}, x\right) \underset{|x| \rightarrow \infty}{=} O\left(e^{-\operatorname{Im} k_{1}|x|}\right), \quad t \in \mathbb{R}
$$


Taking into account

$$
\begin{aligned}
& \pm \int_{x}^{ \pm \infty} d x^{\prime} f_{1, \pm}\left(t, k, x^{\prime}\right)^{2} \\
&= f_{1, \pm}(t, k, x)^{2} \frac{\sigma\left(x-\nu t+\omega^{\prime} \pm 2 b\right) \sigma\left(x-\nu t+\omega^{\prime}\right) \sigma(b)^{2}}{\sigma\left(x-\nu t+\omega^{\prime} \pm b\right)^{2} \sigma(2 b)} \\
& \pm \int_{x}^{ \pm \infty} d x^{\prime} f_{1, \pm}\left(t, k, x^{\prime}\right) f_{1, \pm}\left(t, k_{1}, x^{\prime}\right) \\
&=f_{1, \pm}(t, k, x) f_{1, \pm}\left(t, k_{1}, x\right) \\
& \quad \cdot \frac{\sigma\left(x-\nu t+\omega^{\prime} \pm b \pm b_{1}\right) \sigma\left(x-\nu t+\omega^{\prime}\right) \sigma(b) \sigma\left(b_{1}\right)}{\sigma\left(x-\nu t+\omega^{\prime} \pm b\right) \sigma\left(x-\nu t+\omega^{\prime} \pm b_{1}\right) \sigma\left(b+b_{1}\right)}
\end{aligned}
$$

we get in general

$$
\tilde{f}_{1, \pm}(t, k, x) \underset{x \rightarrow \pm \infty}{=} O\left(f_{1, \pm}(t, k, x)\right) \underset{x \rightarrow \pm \infty}{=} O\left(e^{ \pm i k x}\right),
$$

$$
t \in \mathbb{R},
$$

$$
\begin{aligned}
\tilde{f}_{1, \pm}(t, k, x) \underset{x \rightarrow \mp \infty}{=} 0\left(f_{1, \pm}(t, k, x)\right) \underset{x \rightarrow \mp \infty}{=} O\left(e^{ \pm i k x}\right), \\
k \neq k_{1}, \quad t \in \mathbb{R} .
\end{aligned}
$$

Similarly,

$$
\begin{gathered}
W_{1, \pm}(t, x) \underset{x \rightarrow \pm \infty}{=} O\left(f_{1, \pm}\left(t, k_{1}, x\right)^{2}\right) \underset{x \rightarrow \pm \infty}{=} O\left(e^{\mp 2 \operatorname{Im} k_{1} x}\right), \\
W_{1, \pm}(t, x) \underset{x \rightarrow \mp \infty}{=} 2\left[\mathfrak{P}\left(x-\nu t+\omega^{\prime} \pm 2 b_{1}\right)-\mathfrak{P}\left(x-\nu t+\omega^{\prime}\right)\right] \\
+O\left(e^{ \pm 2 \operatorname{Im} k_{1} x}\right), \quad t \in \mathbb{R} .
\end{gathered}
$$

Remark 10.1. We emphasize the fact that $W_{1,+} \neq W_{1,-}$ and that [76]

$$
W_{1, \pm}(t, x) \underset{x \rightarrow \pm \infty}{\rightarrow} 0, \quad t \in \mathbb{R},
$$

but

$$
W_{1, \pm}(t, x) \underset{x \rightarrow \mp \infty}{\rightarrow} V_{1}\left(t, x \pm 2 b_{1}\right)-V_{1}(t, x) \neq 0, \quad t \in \mathbb{R} .
$$

Thus $W_{1, \pm}(t, x)$ are not decaying as $x \rightarrow \mp \infty$ (as has also been observed in [41]) and hence $W_{1, \pm}(t)$ is no relatively compact perturbation of $H_{1}(t), t \in \mathbb{R}$. However, since, up to exponentially decreasing terms, $V_{1}(t, x)+W_{1, \pm}(t, x)$ is just a transiation $\left(x \rightarrow x \pm 2 b_{1}\right)$ of $V_{1}(t, x)$ as $x \rightarrow \mp \infty$, the band spectrum of $H_{1}(t)$ stays invariant under addition of $W_{1, \pm}(t)$, i.e.,

$$
\sigma_{\text {ess }}\left(\widetilde{H}_{1, \pm}(t)\right)=\sigma\left(H_{1}(t)\right)=\left[E_{0}, E_{1}\right] \cup\left[E_{2}, \infty\right), \quad t \in \mathbb{R} .
$$


Remark 10.2. The special case where $b_{l_{0}}=\bar{b}$ for some $l_{0} \in\{1, \ldots, N\}$ in (10.21) with $\mathfrak{P}(\bar{b})=(2 \omega)^{-1} \int_{0}^{2 \omega} d x \mathfrak{P}\left(x+\omega^{\prime}\right)$ (i.e., $\left.\left(\bar{b}-\omega^{\prime}\right) \in(0, \omega)\right)$ needs a straightforward limiting argument since

$$
F_{z}\left(\lambda_{1}\right)=0, \quad \lambda_{1}:=-\mathfrak{P}(\bar{b})-(\nu / 6) \in\left(E_{1}, E_{2}\right)
$$

(cf. (3.33)), and hence $f_{1, \pm}(t, k(b), x)$ blows up at $b=\bar{b}$.

The results discussed so far are due to [76] (see also [73]). Next we transfer them to the $\mathrm{mKdV}$-case. For simplicity we restrict ourselves to a treatment of $\widetilde{H}_{1,+}(0)$ only. We first discuss the case where $\widetilde{H}_{1,+}(0)$ is critical, i.e., where

$$
\left.\tilde{\mathfrak{E}}_{1}:=\inf \left[\sigma\left(\widetilde{H}_{1,+}(0)\right)\right]=0 \quad \text { (i.e. } \tilde{\lambda}_{1,1}=0\right) .
$$

Then we define

$$
\begin{aligned}
\tilde{\phi}_{0}(t, x):= & -\tilde{f}_{1,+, x}\left(t, k_{0}, x\right) / \tilde{f}_{1,+}\left(t, k_{0}, x\right) \\
= & \phi_{+}(t, x)-\left[1+\tilde{c}_{1,+, 1}(t)^{2} \int_{x}^{+\infty} d x^{\prime} f_{1,+}\left(t, k_{0}, x^{\prime}\right)^{2}\right]^{-1} \\
& \cdot \tilde{c}_{1,+, 1}(t)^{2} f_{1,+}\left(t, k_{0}, x\right)^{2}, \quad k_{0}:=k\left(b_{0}\right), \quad(t, x) \in \mathbb{R}^{2}
\end{aligned}
$$

(cf. (10.28)), with $\phi_{+}(t, x)$ given by (cf. (9.9))

$(10.36)$

$$
\begin{aligned}
\phi_{+}(t, x) & =-f_{1,+, x}\left(t, k_{0}, x\right) / f_{1,+}\left(t, k_{0}, x\right) \\
& =-\frac{1}{2} \frac{\mathfrak{P}^{\prime}\left(x-\nu t+\omega^{\prime}\right)-\mathfrak{P}^{\prime}\left(b_{0}\right)}{\mathfrak{P}\left(x-\nu t+\omega^{\prime}\right)-\mathfrak{P}\left(b_{0}\right)} \\
& =\left[\zeta\left(x-\nu t+\omega^{\prime}\right)+\zeta\left(b_{0}\right)-\zeta\left(x-\nu t+\omega^{\prime}+b_{0}\right)\right], \quad(t, x) \in \mathbb{R}^{2} .
\end{aligned}
$$

Thus

$$
\int_{x_{0}}^{x} d x^{\prime} \tilde{\phi}_{0}\left(t, x^{\prime}\right) \underset{x \rightarrow \pm \infty}{=} \pm \operatorname{Im} k_{0} x+O(1), \quad t \in \mathbb{R}
$$

since $k_{0}=k_{1}$ by assumption. Similarly, in the case where $\widetilde{H}_{1,+}(0)$ is subcritical, i.e., where

$$
\left.\tilde{\mathfrak{E}}_{1}>0 \quad \text { (i.e. } 0<\tilde{\lambda}_{1,1}<E_{0}\right),
$$


we define

$$
\begin{aligned}
\tilde{\phi}_{ \pm}(t, x):=- & \tilde{f}_{1, \pm, x}\left(t, k_{0}, x\right) / \tilde{f}_{1, \pm}\left(t, k_{0}, x\right) \\
=- & \left\{f_{1, \pm}\left(t, k_{0}, x\right) \pm \tilde{c}_{1, \pm, 1}(t)^{2}\right. \\
& \cdot \int_{x}^{ \pm \infty} d x^{\prime}\left[f_{1, \pm}\left(t, k_{0}, x\right) f_{1, \pm}\left(t, k_{1}, x^{\prime}\right)-f_{1, \pm}\left(t, k_{1}, x\right) f_{1, \pm}\left(t, k_{0}, x^{\prime}\right)\right] \\
& \cdot\left\{f_{1, \pm}\left(t, k_{1}, x^{\prime}\right)\right\}^{-1} \\
& \\
& \mp\left[1 \pm \tilde{c}_{1, \pm, 1}\left(t, k_{0}, x\right) \pm \tilde{c}_{1, \pm, 1}(t)^{2} \int_{x}^{ \pm \infty} d x^{\prime}\left[f_{1, \pm, x}\left(t, k_{0}, x\right) f_{1, \pm}\left(t, k_{1}, x^{\prime}\right)\right.\right. \\
& \cdot \tilde{c}_{1, \pm, 1}(t)^{2} f_{1, \pm}\left(t, k_{1}, x\right)^{2}, \quad(t, x) \in \mathbb{R}^{2}
\end{aligned}
$$

(cf. $(10.28)$ ). Thus

$$
\int_{x_{0}}^{x} d x^{\prime} \tilde{\phi}_{ \pm 1}\left(t, x^{\prime}\right) \underset{x \rightarrow \pm \infty}{=} \pm \operatorname{Im} k_{0} x+O(1), \quad t \in \mathbb{R} .
$$

Theorem 7.9 now implies

Theorem 10.3. Let $\tilde{\phi}_{\sigma}, \sigma=0, \pm 1$, be defined by (10.35), resp. (10.39). Then

$$
\operatorname{KdV}\left(\tilde{V}_{2, \sigma}\right)=0, \quad \operatorname{mKdV}\left(\tilde{\phi}_{\sigma}\right)=0, \quad \sigma=0, \pm 1,
$$

where

$$
\widetilde{V}_{2, \sigma}(t, x):=\tilde{\phi}_{\sigma}(t, x)^{2}+\tilde{\phi}_{\sigma, x}(t, x), \quad \sigma=0, \pm 1,(t, x) \in \mathbb{R}^{2} .
$$

Remark 10.4. For $\sigma=0$ one can actually show that

$$
\tilde{V}_{2,0}(t, x)=V_{1}\left(t, x+b_{0}\right), \quad(t, x) \in \mathbb{R}^{2}
$$

(cf. also (9.13)).

\section{Singular SOlutions OF ThE KDV- AND MKDV EQUATIONS}

In this section we indicate how to transfer singular solutions of the $\mathrm{KdV}$ equation to singular solutions of the mKdV-equation using the methods developed in $\S 7$. We also derive rational solutions of the $\mathrm{KdV}$ - and $\mathrm{mKdV}$-equations starting from a pure soliton solution and performing a long-wavelength limit.

We first introduce hypothesis

(H.11.1) Let $x_{0} \in C^{\infty}(\mathbb{R})$ and $X_{0}:=\left\{\left(t, x_{0}(t)\right) \in \mathbb{R}^{2} \mid t \in \mathbb{R}\right\}$.

(i) $V \in C^{\infty}\left(\mathbb{R}^{2} \backslash X_{0}\right)$ real-valued, $\lim _{x \rightarrow \pm \infty} V(t, x)=V_{\infty}, 0<V_{\infty}$ (independent of $t$ ). For each $t \in \mathbb{R}$

$$
\int_{\left|x-x_{0}(t)\right| \geq \varepsilon} d x\left(1+x^{2}\right)\left|V(t, x)-V_{\infty}\right|<\infty
$$


for all sufficiently small $\varepsilon>0$. Furthermore, there exists an open superset $U$ of $X_{0}$ in $\mathbb{R}^{2}$ and a real-valued function $v \in C^{\infty}(U), \partial_{x}^{n} v \in L^{\infty}(U), n=0,1$, such that $V(t, x)=2\left[x-x_{0}(t)\right]^{-2}+v(t, x),(t, x) \in U \backslash X_{0}, \partial_{x} v\left(t, x_{0}(t)\right)=$ $0, t \in \mathbb{R}$, and $\partial_{x}^{n} V \in L^{\infty}\left(\mathbb{R}^{2} \backslash U\right), n=0,1$.

(ii) $\phi \in C^{\infty}\left(\mathbb{R}^{2} \backslash X_{0}\right)$ real-valued, $\lim _{x \rightarrow \pm \infty} \phi(t, x)=\phi_{ \pm}, 0<\phi_{-}^{2}=\phi_{+}^{2}\left(\phi_{ \pm}\right.$ independent of $t)$. For each, $t \in \mathbb{R} \int_{\left|x-x_{0}(t)\right| \geq \varepsilon} d x\left(1+x^{2}\right)\left|\phi(t, x)-\phi_{ \pm}\right|<\infty$ for all sufficiently small $\varepsilon>0$. Furthermore, there is an open superset $U$ of $X_{0}$ in $\mathbb{R}^{2}$ and a real-valued function $\varphi \in C^{\infty}(U), \partial_{x}^{n} \varphi \in L^{\infty}(U), n=0,1$, such that $\phi(t, x)= \pm\left[x-x_{0}(t)\right]^{-1}+\varphi(t, x),(t, x) \in U \backslash X_{0}, \varphi\left(t, x_{0}(t)\right)=$ $0, t \in \mathbb{R}$, and $\partial_{x}^{n} \phi \in L^{\infty}\left(\mathbb{R}^{2} \backslash U\right), n=0,1$.

Remark 11.1. The behavior of $V$ (resp. $\phi$ ) near the singularity $x_{0}(t)$ results from the requirement that the Laurent expansion of $V$ (resp. $\phi$ ) is compatible with the time evolution described by the KdV-equation (resp. mKdVequation).

Theorem 11.2. Let $V_{1}$ denote a solution of $K d V$ which satisfies (H.7.2(i)) with $V_{1+}=V_{1-}=V_{\infty}>0$. Assume $\mathfrak{E}_{1}=\inf \left[\sigma\left(H_{1}\right)\right]>0$ and define

$$
\begin{array}{r}
g_{\gamma}(t, x):=f_{1,+}(t, x, i \beta)-\boldsymbol{r}^{-4 \beta t\left(2 \beta^{2}-3 V_{\infty}\right)} f_{1,-}(t, x, i \beta), \\
(t, x) \in \mathbb{R}^{2}, \gamma \in \mathbb{R},
\end{array}
$$

where $f_{1, \pm}\left(t, x, k_{-}\right)$are Jost solutions corresponding to $V_{1}$ and $\beta=\sqrt{V_{\infty}}$. If, for some $t_{0} \in \mathbb{R}, g_{\gamma}\left(t_{0}, x\right)$ provides precisely one simple zero at $x=x_{0}\left(t_{0}\right)$, then

$$
\phi_{0}(t, x):=-g_{\gamma, x}(t, x) / g_{\gamma}(t, x), \quad(t, x) \in \mathbb{R}^{2} \backslash X_{0},
$$

is a singular solution of the $m K d V$-equation satisfying (H.11.1(ii)), and

$$
V_{2}(t, x):=\phi_{0}(t, x)^{2}+\phi_{0, x}(t, x), \quad(t, x) \in \mathbb{R}^{2} \backslash X_{0},
$$

is a singular solution of the KdV-equation satisfying (H.11.1(i)). In both cases the path of the singularity $x_{0}(t)$ is given by the equation

$$
g_{y}\left(t, x_{0}(t)\right)=0, \quad t \in \mathbb{R} .
$$

Proof. First we note that

$$
f_{1, \pm, t}(t, x, i \beta)=\left\{B_{1}(t) \mp 2 \beta\left(2 \beta^{2}-3 V_{\infty}\right)\right\} f_{1, \pm}(t, x, i \beta), \quad(t, x) \in \mathbb{R}^{2}
$$

(cf. (7.11) for the definition of $\left.B_{1}(t)\right)$. Therefore

$$
\psi_{1}(t, x):=e^{2 \beta t\left(2 \beta^{2}-3 V_{\infty}\right)} g_{\gamma}(t, x), \quad(t, x) \in \mathbb{R}^{2},
$$

is a distributional solution of $H_{1}(t) \psi_{1}(t)=0$ evolving in time according to (7.21). From the assumption that $g_{\gamma}\left(t_{0}, x\right)$ provides precisely one zero at 
$x=x_{0}\left(t_{0}\right)$ we conclude, using Lemma 7.7, that $g_{\gamma}(t, x), t \in \mathbb{R}$, provides precisely one zero at $x=x_{0}(t)$ satisfying (7.32). Due to this fact we may write

$$
\psi_{1}(t, x)=\left(x-x_{0}(t)\right) \chi\left(t,\left(x-x_{0}(t)\right)\right), \quad(t, x) \in \mathbb{R}^{2} .
$$

Inserting (11.7) into (7.21b) and using (7.32) we derive

$$
\chi_{t}\left(t,\left(x-x_{0}(t)\right)\right)=-V_{1, x}(t, x) \chi\left(t,\left(x-x_{0}(t)\right)\right),
$$

and thus

$$
\begin{aligned}
\chi\left(t,\left(x-x_{0}(t)\right)\right)= & \chi\left(t_{0},\left(x-x_{0}\left(t_{0}\right)\right)\right) \\
& \cdot \exp \left[-\int_{t_{0}}^{t} V_{1, x}\left(t^{\prime}, x\right) d t^{\prime}\right], \quad(t, x) \in \mathbb{R}^{2} .
\end{aligned}
$$

Since $\chi\left(t,\left(x-x_{0}(t)\right)\right) \neq 0,(t, x) \in \mathbb{R}^{2}$, the zero remains simple for arbitrary $t$. Using $\psi_{1, x x}=V_{1} \psi_{1}$, it is easily shown that $V_{2}$ (resp. $\phi_{0}$ ) satisfies (H.11.1(i)) (resp. (H.11.1(ii))). Either by direct calculation, using (11.5), or by a generalization of Theorem 7.9, one finally proves that $\operatorname{KdV}\left(V_{2}\right)=0$ and $\operatorname{mKdV}\left(\phi_{0}\right)=0$.

In the following lemma we will exhibit a rich class of potentials $V_{1}$ with $g_{\gamma}\left(t_{0}, \cdot\right)$ providing precisely one simple zero:

Lemma 11.3. Assume $V_{1}$ satisfies (H.7.2(i)) with $V_{+}=V_{-}=V_{\infty}>0$ and either $V_{1}\left(t_{0}, x\right) \geq V_{\infty}, x \in \mathbb{R}$, or $V_{1}$ supports precisely $N$ discrete eigenvalues $\lambda_{N}<\lambda_{N-1}<\cdots<\lambda_{1}, \quad N \in \mathbb{N}$. Then for $0<\lambda_{N}<V_{\infty}$ the function $g_{\gamma}\left(t_{0}, \cdot\right)$ defined in (11.1) with $\beta=\sqrt{V_{\infty}}, \gamma>0$, has precisely one simple zero.

Proof. In what follows we will neglect the argument $t_{0}$. From the integral equation

$f_{1, \pm}(x, i \beta)=e^{\mp \beta x}+\frac{1}{\beta} \int_{x}^{ \pm \infty} d y \sinh (\beta(y-x))\left[V_{1}(y)-V_{\infty}\right] f_{1, \pm}(y, i \beta)$, $x \in \mathbb{R}$,

we infer in case $V_{1}(x) \geq V_{\infty}, x \in \mathbb{R}$, that $f_{1,+}(x, i \beta)\left(f_{1,-}(x, i \beta)\right)$ are positive, strictly monotonically decreasing (increasing) functions, continuously differentiable w.r.t. $x \in \mathbb{R}$. In case $V_{1}$ supports $N$ bound states $\lambda_{1}, \ldots, \lambda_{N}$, $N \geq 1$, let us start with $V_{1}(x ;-1)$ (cf. Theorem 6.5) providing $(N-1)$ bound states $\lambda_{1}, \ldots, \lambda_{N-1}$. Let $f_{1, \pm}\left(x, k_{-} ;-1\right)$ denote the corresponding Jost solutions. Adding the $N$ th bound state $V_{\infty}-\kappa_{N}^{2}=\lambda_{N}$ to obtain $V_{1}$ the Jost solutions corresponding to $V_{1}$ may be written as

$$
f_{1, \pm}\left(x, k_{-}\right)= \pm \frac{1}{i k_{-}-\kappa_{N}} \frac{1}{\tilde{g}_{\alpha}\left(x, i \kappa_{N}\right)} W\left(\tilde{g}_{\alpha}\left(x, i \kappa_{N}\right), f_{1, \pm}\left(x, k_{-} ;-1\right)\right) \text {, }
$$


with

$$
\tilde{g}_{\alpha}\left(x, k_{-}\right)=f_{1,+}\left(x, k_{-} ;-1\right)+\alpha f_{1,-}\left(x, k_{-} ;-1\right), \quad x \in \mathbb{R},
$$
and $\alpha>0$ suitably chosen [33]. For $\beta>\kappa_{N}$ and $\gamma>0$ we thus obtain $(11.13)$

$$
\begin{aligned}
& g_{\gamma}(x)= f_{1,+}(x, i \beta)-\gamma f_{1,-}(x, i \beta)=-\frac{1}{\kappa_{n}+\beta} \frac{1}{\tilde{g}_{\alpha}\left(x, i \kappa_{n}\right)} \\
& \cdot W\left(\tilde{g}_{\alpha}\left(x, i \kappa_{N}\right), f_{1,+}(x, i \beta ;-1)+\gamma f_{1,-}(x, i \beta ;-1)\right) \\
&=\left(\beta-\kappa_{N}\right) \frac{1}{\tilde{g}_{\alpha}\left(x, i \kappa_{N}\right)}\left[\int_{x}^{\infty} \tilde{g}_{\alpha}\left(y, i \kappa_{N}\right) f_{1,+}(y, i \beta ;-1) d y\right. \\
&\left.-\gamma \int_{-\infty}^{x} \tilde{g}_{\alpha}\left(y, i \kappa_{N}\right) f_{1,-}(y, i \beta ;-1) d y\right], \\
& x \in \mathbb{R} .
\end{aligned}
$$

Again we observe that the right-hand side is the difference of a positive, strictly monotonically decreasing and a positive, strictly monotonically increasing function, which in addition is continuously differentiable w.r.t. $x \in \mathbb{R}$. Simplicity of the zero of $g_{\gamma}$ is easily verified in both cases.

Lemma 11.4. Assume that $V_{1}$ is an $N$-soliton solution of the $K d V$-equation, i.e., that, according to (6.29), (6.30),

$$
V_{1}(t, x)=V_{\infty}-2 \partial_{x}\left[\frac{\partial_{x}\left[W\left(a_{1}(t, x), \ldots, a_{N}(t, x)\right)\right]}{W\left(a_{1}(t, x), \ldots, a_{N}(t, x)\right)}\right], \quad(t, x) \in \mathbb{R}^{2},
$$

with

$$
\begin{aligned}
a_{j}(t, x)=(-1)^{j+1} e^{-\kappa_{j} x}+\alpha_{j} e^{-4 \kappa_{j} t\left(2 \kappa_{j}^{2}-3 V_{\infty}\right)} e^{\kappa_{j} x}, \\
j=1, \ldots, N,(t, x) \in \mathbb{R}^{2},
\end{aligned}
$$

and $\kappa_{j}=\sqrt{V_{\infty}-\lambda_{j}}, \alpha_{j}, j=1, \ldots, N$, positive constants. Then the singular solutions of the $K d V$-and $m K d V$-equations obtained in Theorem 11.1 are given by

$$
\begin{array}{r}
V_{2}(t, x)=V_{\infty}-2 \partial_{x}\left[\frac{\partial_{x}\left[W\left(a_{1}(t, x), \ldots, a_{N}(t, x), a_{N+1}(t, x)\right)\right]}{W\left(a_{1}(t, x), \ldots, a_{N}(t, x), a_{N+1}(t, x)\right)}\right], \\
(t, x) \in \mathbb{R}^{2} \backslash X_{0},
\end{array}
$$

and

$$
\begin{aligned}
\phi_{0}(t, x)= & \frac{\partial_{x}\left[W\left(a_{1}(t, x), \ldots, a_{N}(t, x)\right)\right]}{W\left(a_{1}(t, x), \ldots, a_{N}(t, x)\right)} \\
& -\frac{\partial_{x}\left[W\left(a_{1}(t, x), \ldots, a_{N}(t, x), a_{N+1}(t, x)\right)\right]}{W\left(a_{1}(t, x), \ldots, a_{N}(t, x), a_{N+1}(t, x)\right)}, \quad(t, x) \in \mathbb{R}^{2} \backslash X_{0},
\end{aligned}
$$


respectively, where $a_{N+1}$ is defined by (11.15) with $\kappa_{N+1}=\beta=\sqrt{V_{\infty}}$ and $\alpha_{N+1}=-\gamma . V_{2}$ is an $N$-soliton plus one pole solution of the KdV-equation, $\phi_{0}$ is a $2 \mathrm{~N}$-soliton plus one pole solution of the $m K d V$-equation.

Proof. The lemma is proved by using (11.2), (11.3), and (cf. [33])

$$
\begin{aligned}
& f_{1, \pm}(t, x, i \beta)=(\mp 1)^{N} \frac{W\left(a_{1}, \ldots, a_{N}, e^{\mp \beta x}\right)}{\prod_{j=1}^{N}\left(\beta+\kappa_{j}\right) W\left(a_{1}, \ldots, a_{N}\right)} \\
&(t, x) \in \mathbb{R}^{2} .
\end{aligned}
$$

Remark 11.5. We note that the only difference between the $N$-soliton +1 -pole solution (11.16) and an (N+1)-soliton solution (cf. (11.14)) consists in the fact that $\alpha_{N+1}$ is negative in the first case, whereas it is positive in the second. By means of relation (6.33) the constants $\alpha_{i}$ are connected with the norming constants $c_{ \pm, j}^{2}$. Thus, formally, adding a pole to a solution of $\mathrm{KdV}$ corresponds to introducing a "bound state" with negative norming constant $c_{ \pm, N+1}^{2}$ in the associated isospectral Schrödinger operator. A more detailed account on the isospectral problem in connection with singular solutions of the $\mathrm{KdV}$-equation may be found in [9].

Example 11.6. (i) 1-pole solution of the $\mathrm{KdV}$ - and $\mathrm{mKdV}$-equations, $\beta>$ $0, \alpha_{1}=-1$ :

$$
\begin{aligned}
& V_{1}(t, x)=\beta^{2}, \quad(t, x) \in \mathbb{R}^{2}, \\
& V_{2}(t, x)=\beta^{2}+2 \beta^{2} \sinh ^{-2}\left(\beta x+2 \beta^{3} t\right), \quad(t, x) \in \mathbb{R}^{2} \backslash X_{0}, \\
& \phi_{0}(t, x)=-\beta \operatorname{coth}\left(\beta x+2 \beta^{3} t\right), \quad(t, x) \in \mathbb{R}^{2} \backslash X_{0}, \\
& x_{0}(t)=-2 \beta^{2} t, \quad t \in \mathbb{R} .
\end{aligned}
$$

(ii) 1-soliton plus 1-pole solution of the $\mathrm{KdV}$-equation $\beta>\kappa>0, \alpha_{1}=$ $-\alpha_{2}=1$ :

$$
\begin{gathered}
V_{1}(t, x)=\beta^{2}-2 \kappa^{2} \cosh ^{-2}\left(\kappa x-2 \kappa t\left(2 \kappa^{2}-3 \beta^{2}\right)\right), \quad(t, x) \in \mathbb{R}^{2}, \\
\begin{array}{r}
V_{2}(t, x)=\beta^{2}-4\left(\beta^{2}-\kappa^{2}\right)\left[\left(\beta^{2}-\kappa^{2}\right)+\beta^{2} \cosh \left(2 \kappa x-4 \kappa t\left(2 \kappa^{2}-3 \beta^{2}\right)\right)\right. \\
\left.-x^{2} \cosh \left(2 \beta x+4 \beta^{3} t\right)\right] \\
\cdot\left[(\beta-\kappa) \sinh \left((\beta+\kappa) x-4\left(\beta^{3}+\kappa^{3}\right) t+6 \beta^{2}(\beta+\kappa) t\right)\right. \\
\left.+(\beta+\kappa) \sinh \left((\beta-\kappa) x-4\left(\beta^{3}-\kappa^{3}\right) t+6 \beta^{2}(\beta-\kappa) t\right)\right]^{-2}, \\
\quad(t, x) \in \mathbb{R}^{2} \backslash X_{0}, \\
\kappa \tanh \left(\kappa x_{0}(t)-4 \kappa^{3} t+6 \kappa \beta^{2} t\right)=\beta \tanh \left(\beta x_{0}(t)+2 \beta^{3}(t)\right), \quad t \in \mathbb{R} .
\end{array}
\end{gathered}
$$


(iii) 2-soliton plus 1-pole solution of the $\mathrm{mKdV}$-equation, $\beta>\kappa>0, \alpha_{1}=$ $-\alpha_{2}=1$ :

(11.21)

$$
\begin{aligned}
\phi_{0}(t, x)= & \tanh \left(\kappa x-2 \kappa t\left(2 \kappa^{2}-3 \beta^{2}\right)\right) \\
& -2\left(\beta^{2}-\kappa^{2}\right)\left[\cosh \left(\beta x+2 \beta^{3} t\right) \cosh \left(\kappa x-2 x t\left(2 \kappa^{2}-3 \beta^{2}\right)\right)\right] \\
& \cdot\left[(\beta-\kappa) \sinh \left((\beta+\kappa) x-4\left(\beta^{3}+\kappa^{3}\right) t+6 \beta^{2}(\beta+\kappa) t\right)\right. \\
& \left.+(\beta+\kappa) \sinh \left((\beta-\kappa) x-4\left(\beta^{3}-\kappa^{3}\right) t+6 \beta^{2}(\beta-\kappa) t\right)\right]^{-1}, \\
& (t, x) \in \mathbb{R}^{2} \backslash X_{0}, x_{0}(\cdot) \text { as given in }(11.20) .
\end{aligned}
$$

Remark 11.7. The singular solutions in (11.19) formally may be recovered from the one-soliton solutions of $(8.32)$ by choosing the phase $\eta_{1}=\frac{1}{2} \ln \left(c_{1,+, 1}^{2} / 2 \kappa_{1}\right)$ to be $\eta_{1}=i \pi / 2$ instead of $\eta_{1}=0$. Similarly the singular solutions (11.20) and (11.21) formally could have been obtained from two- or three-soliton solutions, respectively, by appropriately choosing the phase constants. In general, the fact that one can recover singular solutions from regular ones relies on the observation that the norming constants $c_{j, \pm}^{2}$ merely enter as parameters, which essentially determine the phase of the $j$ th soliton (see, e.g., [2]).

Exploiting this observation, we finally sketch a derivation of rational solutions of the $\mathrm{KdV}$ - and $\mathrm{mKdV}$-equations. The simplest one is easily obtained from (11.19) by performing the "long-wave" limit $\beta \rightarrow 0$.

Example 11.8. Rational (stationary) solutions of the $\mathrm{KdV}$ - and $\mathrm{mKdV}$-equations:

$$
\begin{array}{ll}
V_{1}(t, x)=0, & (t, x) \in \mathbb{R}^{2}, \\
V_{2}(t, x)=\frac{2}{x^{2}}, & (t, x) \in \mathbb{R}^{2} \backslash X_{0}, \\
\phi_{0}(t, x)=-\frac{1}{x}, & (t, x) \in \mathbb{R}^{2} \backslash X_{0}, X_{0}=\mathbb{R} \times\{0\} .
\end{array}
$$

In order to demonstrate how more general rational solutions of the $\mathrm{KdV}$ - and $\mathrm{mKdV}$-equations may be obtained from the corresponding soliton solutions it suffices here to consider the two-soliton solution of the $\mathrm{KdV}$-equation which we write in the form

$$
V_{1}(t, x)=\kappa_{2}^{2}-2 \partial_{x}^{2}\left\{\ln \left[W\left(a_{1}(t, x), a_{2}(t, x)\right)\right]\right\}, \quad(t, x) \in \mathbb{R}^{2},
$$

with $\kappa_{2}>\kappa_{1}>0$. Here $a_{j}, j=1,2$, are defined via (11.15) with $V_{\infty}=\kappa_{2}^{2}$ and $\alpha_{j}$ are related to the norming constants $c_{j,+}, j=1,2$, via (6.33). The potential $V_{2,0}$ then is given by (see Theorem 8.1)

$$
V_{2,0}(t, x)=\kappa_{2}^{2}-2 \partial_{x}^{2}\left\{\ln \left[a_{1}(t, x)\right]\right\}, \quad(t, x) \in \mathbb{R}^{2},
$$

and furthermore (cf. (7.63))

$$
\phi_{0}(t, x)=\partial_{x}\left\{\ln \left[W\left(a_{1}(t, x), a_{2}(t, x)\right) / a_{1}(t, x)\right]\right\}, \quad(t, x) \in \mathbb{R}^{2}
$$


Now taking $\alpha_{j}=(-1)^{j}, j=1,2$, and performing the "long-wave" limit $\kappa_{1}, \kappa_{2} \rightarrow 0$ one obtains

Example 11.9.

$$
\begin{aligned}
V_{1}(t, x) & =-2 \partial_{x}^{2} \ln \left(x^{3}+12 t\right) \\
& =\frac{18 x^{4}}{\left(x^{3}+12 t\right)^{2}}-\frac{12 x}{\left(x^{3}+12 t\right)}, \quad(t, x) \in \mathbb{R}^{2} \backslash X_{0}, \\
V_{2}(t, x) & =-2 \partial_{x}^{2} \ln (x)=\frac{2}{x^{2}}, \quad(t, x) \in \mathbb{R}^{2} \backslash\{\mathbb{R} \times\{0\}\}, \\
\phi_{0}(t, x) & =\partial_{x}\left[\ln \left(x^{3}+12 t\right)-\ln (x)\right] \\
& =\frac{2 x^{3}-12 t}{x\left(x^{3}+12 t\right)}, \quad(t, x) \in \mathbb{R}^{2} \backslash X_{0}, \\
x_{0}(t) & =-\operatorname{sgn}(t)|12 t|^{1 / 3}, \quad t \in \mathbb{R}(\operatorname{sgn}(0):=0) .
\end{aligned}
$$

Whereas these rational solutions of the $\mathrm{KdV}$-equation are well known [1, 2, $6,22,42,53,81]$ the corresponding solutions of the $\mathrm{mKdV}$-equation seem to be new (although one could easily derive them from the results in [6]).

Acknowledgments. We are indebted to David Gurarie for several most stimulating discussions. F. Gesztesy is indebted to D. Wales for the hospitality extended to him at Caltech. He also gratefully acknowledges financial support from the Max Kade Foundation and from USNSF under Grant DMS-8801918.

\section{REFERENCES}

1. M. J. Ablowitz and H. Cornille, On solutions of the Korteweg-de Vries equation, Phys. Lett. A 72 (1979), 277-280.

2. M. J. Ablowitz and J. Satsuma, Solitons and rational solutions of nonlinear evolution equations, J. Math. Phys. 19 (1978), 2180-2186.

3. M. J. Ablowitz, M. Kruskal, and H. Segur, A note on Miura's transformation, J. Math. Phys. 20 (1979), 999-1003.

4. M. J. Ablowitz, D. J. Kaup, A. C. Newell, and H. Segur, The inverse scattering transformFourier analysis for nonlinear problems, Stud. Appl. Math. 53 (1974), 249-315.

5. M. Abramowitz and I. A. Stegun, Handbook of mathematical functions, Dover, New York, 1972.

6. M. Adler and J. Moser, On a class of polynomials connected with the Korteweg-de Vries equation, Comm. Math. Phys. 61 (1978), 1-30.

7. M. Antonowicz and A. Fordy, Factorization of energy dependent Schrödinger operators: Miura maps and modified systems, Comm. Math. Phys. 124 (1989), 465-486.

8. A. Arai, Some remarks on scattering theory in supersymmetric quantum mechanics, J. Math. Phys. 28 (1987), 472-476.

9. V. A. Arkad'ev, A. K. Pogrebkov, and M. K. Polivanov, Singular solutions of the KdV equation and the inverse scattering method, J. Soviet Math. 31 (1985), 3264-3279.

10. T. C. Au-Yeung, C. Au, and P. C. W. Fung, One soliton Korteweg-de Vries solution with nonzero vacuum parameters obtainable from the generalized inverse scattering method, Phys. Rev. A 29 (1984), 2370-2374. 
11. T. C. Au-Yeung, P. C. W. Fung, and C. Au, Modified $K d V$ solitons with non-zero vacuum parameter obtainable from the ZS-AKNS inverse method, J. Phys. A 17 (1984), 1425-1436.

12. R. Beals, P. Deift, and C. Tomei, Direct and inverse scattering on the line, Mathematical Surveys and Monographs, vol. 28, Amer. Math. Soc., Providence, R. I., 1988.

13. M. J. Bergvelt and A. P. E. ten Kroode, $\tau$ functions and zero curvature equations of the Toda-AKNS type, J. Math. Phys. 29 (1988), 1308-1320.

14. D. Bollé, F. Gesztesy,and M. Klaus, Scattering theory for one-dimensional systems with $\int d x V(x)=0$, J. Math. Anal. Appl. 122 (1987), 496-518.

15. D. Bollé, F. Gesztesy, and S. F. J. Wilk, A complete treatment of low-energy scattering in one dimension, J. Operator Theory 13 (1985), 3-31.

16. D. Bollé, F. Gesztesy, H. Grosse, W. Schweiger, and B. Simon, Witten index, axial anomaly, and Krein's spectral shift function in supersymmetric quantum mechanics, J. Math. Phys. 28 (1987), 1512-1525.

17. N. V. Borisov, W. Müller, and R. Schrader, Relative index theorems and supersymmetric scattering theory, Comm. Math. Phys. 114 (1988), 475-513.

18. M. Bruschi and F. Calogero, The Lax representation for an integrable class of relativistic dynamical systems, Comm. Math. Phys. 109 (1987), 481-492.

19. J. L. Burchnall and T. W. Chaundy, Commutative ordinary differential operators, Proc. Roy. Soc. London Ser. A 118 (1928), 557-583.

20. _ Commutative ordinary differential operators II. The identity $P^{n}=Q^{m}$, Proc. Roy. Soc. London Ser. A 134 (1931-32), 471-485.

21. M. Buys and A. Finkel, The inverse periodic problem for Hill's equation with a finite-gap potential, J. Differential Equations 55 (1984), 257-275.

22. F. Calogero and A. Degasperis, Spectral transforms and solitons, Vol. 1, North-Holland, Amsterdam, 1982.

23. R. Carmona, One-dimensional Schrödinger operators with random or deterministic potentials: New spectral types, J. Funct. Anal. 51 (1983), 229-258.

24. S. Chaturvedi and $\mathrm{K}$. Raghunathan, Relation between the Gelfand-Levitan procedure and the method of supersymmetric partners, J. Phys. A 19 (1986), L775-L778.

25. A. Cohen, Decay and regularity in the inverse scattering problem, J. Math. Anal. Appl. 87 (1982), 395-426.

26. __ Solutions of the Korteweg-De Vries equation with steplike initial profile, Comm. Partial Differential Equations 9 (1984), 751-806.

27. A. Cohen and T. Kappeler, Scattering and inverse scattering for steplike potentials in the Schrödinger equation, Indiana Univ. Math. J. 34 (1985), 127-180.

28. M. M. Crum, As! ciated Sturm-Liouville systems, Quart. J. Math. Oxford (2) 6 (1955), 121-127.

29. G. Darboux, Sur une proposition relative aux équations linéaires, C.R. Acad. Sci. (Paris) 94 (1882), 1456-1459.

30. E. Date and S. Tanaka, Periodic multi-soliton solutions of Korteweg-de Vries equation and Toda lattice, Suppl. Progr. Theoret. Phys. 59 (1976), 107-125.

31. E. B. Davies and B. Simon, Scattering theory for systems with different spatial asymptotics on the left and right, Comm. Math. Phys. 63 (1978), 277-301.

32. P. A. Deift, Applications of a commutation formula, Duke Math. J. 45 (1978), 267-310.

33. P. Deift and E. Trubowitz, Inverse scattering on the line, Comm. Pure Appl. Math. 32 (1979), 121-251.

34. R. K. Dodd, J. C. Eilbeck, J. D. Gibbon, and H. C. Morris, Solitons and nonlinear wave equations, Academic Press, New York, 1982.

35. B. A. Dubrovin, Inverse problem for periodic finite-zoned potentials in the theory of scattering, Functional. Anal. Appl. 9 (1975), 61-62. 
36. B. A. Dubrovin and S. P. Novikov, Periodic and conditionally periodic analogs of the manysoliton solutions of the Korteweg-de Vries equation, Soviet Phys. JETP 40 (1975), 1058-1063.

37. N. Dunford and J. T. Schwartz, Linear operators. II, Interscience, New York, 1963.

38. M. S. P. Eastham, The spectral theory of periodic differential equations, Scottish Academic Press, Edinburgh, 1973.

39. W. Eckhaus and A. Van Harten, The inverse scattering transformation and the theory of solitons, Math. Studies, no. 50, North-Holland, Amsterdam, 1981.

40. H. Flaschka, On the inverse problem of Hill's operator, Arch. Rational Mech. Anal. 59 (1975), 293-309.

41. H. Flaschka and D. W. McLaughlin, Some comments on Bäcklund transformations, canonical transformations, and the inverse scattering method, Lecture Notes in Math., vol. 516, (R. M. Miura, ed.), Springer-Verlag, Berlin, 1976, pp. 252-295.

42. H. Flaschka and A. C. Newell, Monodromy-and spectrum-preserving deformations. I, Comm. Math. Phys. 76 (1980), 65-116.

43. N. E. Firsova, Riemann surface of quasimomentum and scattering theory for the perturbed Hill operator, J. Soviet Math. 11 (1975), 487-497.

44. _ An inverse scattering problem for a perturbed Hill operator, Math. Notes 18 (1975), 1085-1091.

45. _ Some spectral identities for the one-dimensional Hill operator, Theoret. Math. Phys. 37 (1977), 1022-1027.

46. N. E. Firsova, The direct and inverse scattering problems for the one-dimensional perturbed Hill operator, Math. USSR Sb. 58 (1987), 351-388.

47. P. C. W. Fung and C. Au, Bridge between the solutions and vacuum states of the Korteweg-de Vries equation and that of the nonlinear equation $y_{t}+y_{x x x}-6 y^{2} y_{x}+6 \lambda y_{x}=0$, Phys. Rev. B 26 (1982), 4035-4038.

48. C. S. Gardner, J. M. Greene, M. D. Kruskal, and R. M. Miura, Korteweg-de Vries equation and generalizations, VI. Methods for exact solution, Comm. Pure Appl. Math. 27 (1974), 97-133.

49. F. Gesztesy, Scattering theory for one-dimensional systems with nontrivial spatial asymptotics, in Schrödinger Operators, Aarhus 1985 (E. Balslev, ed.), Lecture Notes in Math., vol. 1218, Springer-Verlag, Berlin, 1986, pp. 93-122.

50. F. Gesztesy and B. Simon, Constructing solutions of the mKdV-equation, J. Funct. Anal. (in print).

51. J. Ginibre and Y. Tsutsumi, G. Velo, Existence and uniqueness of solutions for the generalized Korteweg-de Vries equation, SIAM J. Math. Anal. 20 (1989), 1388-1425.

52. W. Goldberg and H. Hochstadt, On a Hill's equation with selected gaps in its spectrum, J. Differential Equations 34 (1979), 167-178.

53. D. Grecu and V. Cionga, On the mixed soliton-rational solutions of the $\mathrm{KdV}$ equation, preprint, 1986.

54. H. Grosse, Solitons of the modified KdV equation, Lett. Math. Phys. 8 (1984), 313-319.

55. __ New solitons connected to the Dirac equation, Phys. Rep. 134 (1986), 297-304.

56. P. Hartman, Ordinary differential equations Wiley, New York, 1964.

57. R. Hirota, Exact solution of the Korteweg-de Vries equation for multiple collisions of solitons, Phys. Rev. Lett. 18 (1971), 1192-1194.

58. _ Exact solution of the modified Korteweg-de Vries equation for multiple collisions of solitons, J. Phys. Soc. Japan 33 (1972), 1456-1458.

59. H. Hochstadt, On the determination of a Hill's equation from its spectrum, Arch. Rational. Mech. Anal. 19 (1965), 353-362.

60. A. R. Its, Inversion of hyperelliptic integrals and integration of nonlinear differential equations, Vestnik Leningrad Univ. Math. 9 (1981), 121-129. 
61. A. R. Its and V. B. Matveev, Schrödinger operator with finite-gap spectrum and $N$-soliton solutions of the Korteweg-de Vries equation, Theoret. Math. Phys. 23 (1975), 343-355.

62. K. Iwasaki, Inverse problem for Sturm-Liouville and Hill equations, Ann. Mat. Pura Appl. (4) 149 (1987), 185-206.

63. C. G. J. Jacobi, Zur Theorie der Variationsrechnung und der Differentialgleichungen, J. Reine Angew. Math. 17 (1837), 68-82.

64. A. Jaffe, A. Lesniewski and M. Lewenstein, Ground state structure in supersymmetric quantum mechanics, Ann. Phys. 178 (1987), 313-329.

65. M. Jaulent and I. Miodek, Connection between Zakharov-Shabat and Schrödinger-type inverse scattering transform, Lett. Nuovo Cimento 20 (1977), 655-660.

66. Y. Kametaka, Korteweg-de Vries equation III. Global existence of asymptotically periodic solutions, Proc. Japan Acad. 45 (1969), 656-660.

67. __ Korteweg-de Vries equation IV. Simplest generalization, Proc. Japan Acad. 45 (1969), 661-665.

68. T. Kato, On the Korteweg-de Vries equation, Manuscripta Math. 28 (1979), 89-99.

69. __ On the Cauchy problem for the (generalized) Korteweg-de Vries equation, Studies in Applied Mathematics, (V. Guillemin, ed.), Adv. Math. Suppl. Studies, no. 18, Academic Press, New York, 1983, pp. 93-128.

70. Y. Kato, Fredholm determinants and the Cauchy problem of a class of nonlinear evolution equations, Progr. Theoret. Phys. 78 (1987), 198-213.

71. J. Kay and H. E. Moses, Reflectionless transmission through dielectrics and scattering potentials, J. Appl. Phys. 27 (1956), 1503-1508.

72. M. Klaus, Low-energy behavior of the scattering matrix for the Schrödinger equation on the line, Inverse Problems 4 (1988), 505-512.

73. I. M. Krichever, Potentials with zero coefficient of reflection on a background of finite-zone potentials, Functional Anal. Appl. 9 (1975), 161-163.

74. S. N. Kruzhkov and A. V. Faminskii, Generalized solutions of the Cauchy problem for the Korteweg-de Vries equation, Math. USSR Sb. 48 (1984), 391-421.

75. B. A. Kupershmidt and G. Wilson, Modifying Lax equations and the second Hamiltonian structure, Invent. Math. 62 (1981), 403-436.

76. E. A. Kuznetsov and A. V. Mikhailov, Stability of stationary waves in nonlinear weakly dispersive media, Soviet Phys. JETP 40 (1975), 855-859.

77. P. D. Lax, Integrals of nonlinear equations of evolution and solitary waves, Comm. Pure Appl. Math. 21 (1968), 467-490.

78. _ _ Periodic solutions of the $K d V$ equations, Lectures in Appl. Math. 15 (1974), 85-96.

79. W. Magnus and S. Winkler, Hill's equation, Dover, New York, 1979.

80. V. A. Marchenko, Sturm-Liouville operators and applications", Birkhäuser, Basel, 1986.

81. H. P. McKean, Theta functions, solitons, and singular curves, Partial Differential Equations and Geometry (C. I. Byrnes, ed.), Marcel Dekker, 1979, pp. 237-254.

82. H. P. McKean, Geometry of $\mathrm{KdV}(1)$ : Addition and the unimodular spectral classes, Rev. Mat. Iberoamericana 2 (1986), 235-261.

83. _ Geometry of $\operatorname{KdV}(2)$ : Three examples, J. Statist. Phys. 46 (1987), 1115-1143.

84. H. P. McKean and P. van Moerbeke, The spectrum of Hill's equation, Invent. Math. 30 (1975), 217-274.

85. H. P. McKean and E. Trubowitz, Hills operator and hyperelliptic function theory in the presence of infinitely many branch points, Comm. Pure Appl. Math. 29 (1976), 143-226.

86. __ Hill's surfaces and their theta functions, Bull. Amer. Math. Soc. 84 (1978), 1042-1085.

87. A. Menikoff, The existence of unbounded solutions of the Korteweg-de Vries equation, Comm. Pure Appl. Math. 25 (1972), 407-432. 
88. R. M. Miura, Korteweg-de Vries equation and generalizations, I. A remarkable explicit nonlinear transformation, J. Math. Phys. 9 (1968), 1202-1204.

89. H. E. Moses, A generalization of the Gelfand-Levitan equation for the one-dimensional Schrödinger equation, J. Math. Phys. 18 (1977), 2243-2250.

90. M. Murata, Structure of positive solutions to $(-\Delta+V) u=0$ in $\mathbb{R}^{n}$, Duke Math. J. 53 (1986), 869-943.

91. M. M. Nieto, Relationship between supersymmetry and the inverse method in quantum mechanics, Phys. Lett. B 145 (1984), 208-210.

92. S. P. Novikov, The periodic problem for the Korteweg-de Vries equation, Functional Anal. Appl. 8 (1974), 236-246.

93. S. Novikov, S. V. Manakov, L. P. Pitaevskii, and V. E. Zakharov, Theory of solitons, Consultants Bureau, New York, 1984.

94. M. Ohmiya, On the generalized soliton solutions of the modified Korteweg-de Vries equation, Osaka J. Math. 11 (1974), 61-71.

95. M. Reed and B. Simon, Methods of modern mathematical physics IV: Analysis of operators, Academic Press, New York, 1978.

96. S. N. M. Ruijsenaars and P. J. M. Bongaarts, Scattering theory for one-dimensional step potentials, Ann. Inst. H. Poincaré A 26 (1977), 1-17.

97. S. N. M. Ruijsenaars and H. Schneider, A new class of integrable systems and its relation to solitons, Ann. Phys. 170 (1986), 370-405.

98. D. H. Sattinger and V. D. Zurkowski, Gauge theory of Bäcklund transformations II, Phys. D 26 (1987), 225-250.

99. U.-W. Schmincke, On Schrödinger's factorization method for Sturm-Liouville operators, Proc. Roy. Soc. Edinburgh Sect. A 80 (1978) , 67-84.

100. B. Simon, Large time behavior of the $L^{P}$ norm of Schrödinger semigroups, J. Funct. Anal. 40 (1981), 66-83.

101. A. Sjöberg, On the Korteweg-de Vries equation: Existence and uniqueness, J. Math. Anal. Appl. 29 (1970), 569-579.

102. V. V. Sokolov and A. B. Shabat, $(L, A)$-pairs and a Riccati type substitution, Functional Anal. Appl. 14 (1980), 148-150.

103. O. Steinmann, Äquivalente periodische Potentiale, Helv. Phys. Acta 30 (1957), 515-520.

104. C. V. Sukumar, Supersymmetric quantum mechanics of one-dimensional systems, J. Phys. A 18 (1985), 2917-2936.

105. S. Tanaka, Modified Korteweg-de Vries equation and scattering theory, Proc. Japan Acad. 48 (1972), 466-469.

106. S. Tanaka, On the N-tuple wave solutions of the Korteweg-de Vries equation, Publ. Res. Inst. Math. Soc. 8 (1972/73), 419-427.

107. S. Tanaka, Some remarks on the modified Korteweg-de Vries equation, Publ. Res. Inst. Math. Sci. 8 (1972/73), 429-437.

108. S. Tanaka, Non-linear Schrödinger equation and modified Korteweg-de Vries equation; construction of solutions in terms of scattering data, Publ. Res. Inst. Math. Soc. 10 (1975), 329-357.

109. B. Thaller, Normal forms of an abstract Dirac operator and applications to scattering theory, J. Math. Phys. 29 (1988), 249-257.

110. E. Trubowitz, The inverse problem for periodic potentials, Comm. Pure Appl. Math. 30 (1977), 321-337.

111. M. Tsutsumi, On global solutions of the generalized Korteweg-de Vries equation, Publ. Res. Inst. Math. Soc. 7 (1972), 329-344.

112. S. Venakides, The zero dispersion limit of the Korteweg-de Vries equation with periodic initial data, Trans. Amer. Math. Soc. 301 (1987), 189-226. 
113. M. Wadati, The exact solution of the modified Korteweg-de Vries equation, J. Phys. Soc. Japan 32 (1972), 1681.

114. __ The modified Korteweg-de Vries equation, J. Phys. Soc. Japan 34 (1973), 1289-1296.

115. M. Wadati and M. Toda, The exact $N$-soliton solution of the Korteweg-de Vries equation, J. Phys. Soc. Japan 32 (1972), 1403-1411.

116. H. D. Wahlquist, Bäcklund transformation of potentials of the Korteweg-de Vries equation and the interaction of solitons with cnoidal waves, Lecture Notes in Math., vol. 515 (R. M. Miura, ed.), Springer-Verlag, Berlin, 1976, pp. 162-183.

117. H. D. Wahlquist and F. B. Estabrook, Prolongation structures of nonlinear evolution equations, J. Math. Phys. 16 (1975), 1-7.

118. G. Wilson, Infinite-dimensional Lie groups and algebraic geometry in soliton theory, Philos. Trans. Roy. Soc. London Ser. A 315 (1985), 393-404.

119. Yu-kun Zheng and W. L. Chan, Gauge transformation and the higher order Korteweg-de Vries equation, J. Math. Phys. 29 (1988), 308-314.

Department of Mathematics, University of Missouri, Columbia, Missouri 65211

FB8-Naturwissenschaften I, Theoretische Physik, Bergische Universität, Gesamthochschule, D-5600 Wuppertal 1, Federal Republic of Germany

Division of Physics, Mathematics and Astronomy, California Institute of Technology, Pasadena, California 91125 